(c) 2004 International Press

Adv. Theor. Math. Phys. 8 (2004) 1-82

\title{
Matrix Quantum Mechanics and Soliton Regularization of Noncommutative Field Theory
}

\author{
Giovanni Landi ${ }^{1,2}$, Fedele Lizzi ${ }^{2,3,4}$ and Richard J. Szabo ${ }^{4}$ \\ ${ }^{1}$ Dipartimento di Scienze Matematiche, Università di Trieste \\ Via A. Valerio 12/1, I-34127 Trieste, Italia \\ ${ }^{2}$ INFN, Sezione di Napoli, Napoli, Italia \\ 3 Dipartimento di Scienze Fisiche, Università di Napoli Federico II \\ Monte S. Angelo, Via Cintia, 80126 Napoli, Italia \\ ${ }^{4}$ Department of Mathematics, Heriot-Watt University \\ Scott Russell Building, Riccarton, Edinburgh EH14 4AS, U.K.
}

landi@univ.trieste.it, fedele.lizzi@na.infn.it , R.J.Szabo@ma.hw.ac.uk

e-print archive:

http://lanl.arXiv.org/abs/hep-th/0401072 


\begin{abstract}
We construct an approximation to field theories on the noncommutative torus based on soliton projections and partial isometries which together form a matrix algebra of functions on the sum of two circles. The matrix quantum mechanics is applied to the perturbative dynamics of scalar field theory, to tachyon dynamics in string field theory, and to the Hamiltonian dynamics of noncommutative gauge theory in two dimensions. We also describe the adiabatic dynamics of solitons on the noncommutative torus and compare various classes of noncommutative solitons on the torus and the plane.
\end{abstract}

\title{
1 Introduction and Summary
}

Among the principal characteristics of noncommutative spaces $[17,44,53$, 31 , whichever way we may choose to define them, is the fact that the concept of locality becomes evanescent and disappears altogether. Noncommutativity typically introduces a length scale below which it is no longer possible to resolve "points" in the space. If a noncommutative space cannot be described by the local fields defined on it, it is still possible to use those fields, which technically live in a noncommutative $C^{*}$-algebra, to describe some geometric properties of the space. In some instances, for example when the noncommutative spaces are deformations of ordinary ones, it may still be possible to "see" the points underlying the algebra, and the noncommutativity is typically described by the nonvanishing commutator of coordinates. This description may be appealing for the connections which can be made with ordinary geometry, but it does hide some novel characteristics of noncommutative geometry which can have important physical implications and provide useful calculational tools. For instance, there exist solitonic solutions in noncommutative geometry which have no counterparts in commutative geometry [29]. By solitons we mean nonvanishing finite energy extrema of the action functional of a given field theory, and in the examples to be considered in this paper they are described by projections or partial isometries of the underlying noncommutative algebra. In the following we will in fact use the words solitons and projections/partial isometries synonymously.

One of the main physical interests in noncommutative geometry is the fact that it arises naturally in string theory, and in particular the noncommutative torus $[68,16]$ describes naturally the stringy modifications to classical spacetime [19, 45, 72] (see [42, 24, 75] for reviews). In the context of open string field theory, the algebraic structure of noncommutative geometry allows a particularly simple construction of both stable and unstable D-branes 
in terms of projections and partial isometries [23, 38, 78]. Also related to this operatorial nature is the fact that noncommutative field theories can be regulated and studied by means of matrix models $[19,6,4,5,54,11,52,33$, $49,74]$. In the case of field theories on the noncommutative torus, the matrix regularization yields field theories on the fuzzy torus and is intimately related to the lattice regularization of noncommutative field theories $[4,5]$.

Although the matrix model formalisms have many computational advantages, they have several pitfalls as well. Foremost among these are the complicated double scaling limits required to reproduce the original continuum dynamics. The complicated nature is related to the mathematical fact that the algebra of functions on a manifold can never be the exact inductive limit of finite dimensional algebras, and examples abound for which the finite approximations fail to capture relevant physical aspects or produce phenomena which are unphysical artifacts of the matrix regularization. Technically, we may say that no algebra of functions can be an approximately finite (AF) algebra [46]. In this paper we will show how to overcome this problem by exploiting one of the aforementioned novel characteristics of noncommutative field theories, namely the presence of projection operators (or projections for brevity). As we have mentioned, they play an important role in the effective field theories of D-branes in that they are finite energy extrema of the potential energy, or solitons.

In what follows, after a review of the Elliott-Evans construction of the sequence of algebras approximating the noncommutative torus [26], we will construct viable field theories based on it. The interest in this construction is many-fold. The approximate algebras are generated by projections and partial isometries which together generate the direct sum of two copies of the algebra of matrix-valued functions on a circle, and therefore the approximation to a noncommutative field theory is effectively a matrix quantum mechanics which can be solved exactly in some cases. Unlike the usual lattice approximations, the noncommutative torus is the inductive limit of the sequence of algebras in the strong rigorous sense. From a computational point of view, this means that the continuum limit is much simpler. It is important to realize though that it is not simply the 't Hooft planar limit of the matrix model, and the notion of planarity in the matrix quantum mechanics coincides with that of the original noncommutative field theory [60].

We will show that the field theory corresponding to the soliton approximation can be used, as a quantum mechanics, in a quantitatively useful manner for field theoretic calculations. For example, we will analyse in detail the dynamics of a noncommutative scalar field theory and show that ultraviolet-infrared (UV/IR) mixing [60] is cured by the approximation (but 
of course reappears in the limit). We also show that the approximation already captures quantitative aspects of tachyon condensation in string field theory, and further demonstrate how the exact solution of gauge theory on the noncommutative torus [62] is captured by the Hamiltonian dynamics of the matrix quantum mechanics. The approximation presented in this paper thereby has the opportunity to capture important nonperturbative aspects of noncommutative field theories.

We will also study the adiabatic dynamics of projections according to a $\sigma$ model action defined on soliton moduli space. We will find that the extrema of the action are solitons which satisfy a certain self-duality or anti-selfduality condition. The typical soliton of this kind, the Boca projection [14], is the torus equivalent of the GMS solitons on the noncommutative plane [29]. The field configurations correspond to smooth "bump" functions which are localized within the scale of noncommutativity, and they are very different from the projections which generate the matrix algebras. The latter projections generalize the Power-Rieffel projections [68], and the corresponding fields wind around the torus thereby exhibiting a more non-local structure. In the context of tachyon condensation on the two-dimensional noncommutative torus, the Boca projection has been employed in [56, 28, 43, 40, 39] and the Powers-Rieffel projection in $[9,71,56]$. From the dynamically obtained Boca projection we will then use the matrix regularization on the noncommutative torus to induce approximations also of field theories on the noncommutative plane.

\section{Outline}

The structure of the remainder of this paper is as follows. In section 2 we introduce the main characteristics describing field theories on the noncommutative torus, their connection with tachyon condensation, and the sequence of projections which will form the diagonal part of the matrix approximation. In section 3 we describe in detail the construction of the matrix subalgebras and the way the approximation is realized. In section 4 , which is the crux of the paper, we describe how to construct the matrix quantum mechanics equivalent (in the limit) of a generic noncommutative field theory. In section 5 we present three examples of the formalism, involving the perturbative dynamics of $\phi^{4}$ scalar field theory on the noncommutative torus, the construction of D-branes as decay products in tachyon condensation, and a Hamiltonian analysis of noncommutative Yang-Mills theory in two dimensions. In the final section 6 , we describe the relationships between the solitons used for the matrix approximation and the Boca projection, the toroidal generalization of the GMS lump configurations, which leads to the 
planar version of the matrix model regularization. There we also describe the dynamics of solitons on the noncommutative torus through a $\sigma$-model defined on their configuration space. Some technical details are presented in five appendices at the end of the paper. Some aspects of the present paper have been announced in [47].

\section{Solitons on the Noncommutative Torus}

In this section we will review the construction of solitonic field configurations on the two-dimensional noncommutative torus, primarily to introduce the physical notions, the notation and the definitions which will be used throughout this paper. We will begin with a review of the geometry of the noncommutative torus, emphasizing those ingredients which are important for the construction of noncommutative field theories. We shall then briefly review the construction of D-branes as solitons in the effective field theory of open strings, as this will set the main physical motivation for most of our subsequent analysis. Then we will describe an important set of projections for the noncommutative torus.

\subsection{Field Theories on the Noncommutative Torus}

Consider an ordinary square two-torus $\mathbb{T}^{2}$ with coordinate functions $U=$ $\mathrm{e}^{2 \pi \mathrm{i} x}$ and $V=\mathrm{e}^{2 \pi \mathrm{i} y}$, where $x, y \in[0,1]$. By Fourier expansion the algebra $C^{\infty}\left(\mathbb{T}^{2}\right)$ of complex-valued smooth functions on the torus is made up of all power series of the form

$$
a=\sum_{(m, r) \in \mathbb{Z}^{2}} a_{m, r} U^{m} V^{r}
$$

with $\left\{a_{m, r}\right\} \in S\left(\mathbb{Z}^{2}\right)$ a complex-valued Schwartz function on $\mathbb{Z}^{2}$. This means that the sequence of complex numbers $\left\{a_{m, r} \in \mathbb{C} \mid(m, r) \in \mathbb{Z}^{2}\right\}$ decreases rapidly at "infinity", i.e. for any $k \in \mathbb{N}_{0}$ one has bounded semi-norms

$$
\|a\|_{k}=\sup _{(m, r) \in \mathbb{Z}^{2}}\left|a_{m, r}\right|(1+|m|+|r|)^{k}<\infty .
$$

Let us now fix a real number $\theta$. The algebra $\mathcal{A}_{\theta}=C^{\infty}\left(\mathbb{T}_{\theta}^{2}\right)$ of smooth functions on the noncommutative torus is the associative algebra made up of all elements of the form (2.1), but now the two generators $U$ and $V$ satisfy

$$
V U=\mathrm{e}^{2 \pi \mathrm{i} \theta} U V
$$


The algebra $\mathcal{A}_{\theta}$ can be made into a $*$-algebra by defining a $*$-involution $\dagger$ by

$$
U^{\dagger}:=U^{-1}, \quad V^{\dagger}:=V^{-1} .
$$

From (2.2) with $k=0$ one gets a $C^{*}$-norm and the corresponding closure of $\mathcal{A}_{\theta}$ in this norm is the universal $C^{*}$-algebra $A_{\theta}$ generated by two unitaries with the relation $(2.3) ; \mathcal{A}_{\theta}$ is dense in $A_{\theta}$ and is thus a pre- $C^{*}$-algebra.

In the following we shall use the one-to-one correspondence between elements of the noncommutative torus algebra $\mathcal{A}_{\theta}$ and the commutative torus algebra $C^{\infty}\left(\mathbb{T}^{2}\right)$ given by the Weyl map $\Omega$ and its inverse, the Wigner map. As is usual for a Weyl map, there are operator ordering ambiguities, and so we will take the prescription

$$
\Omega\left(\sum_{(m, r) \in \mathbb{Z}^{2}} f_{m, r} \mathrm{e}^{2 \pi \mathrm{i}(m x+r y)}\right):=\sum_{(m, r) \in \mathbb{Z}^{2}} f_{m, r} \mathrm{e}^{\pi \mathrm{i} m r \theta} U^{m} V^{r} .
$$

This choice (called Weyl or symmetric ordering) maps real-valued functions on $\mathbb{T}^{2}$ into Hermitian elements of $\mathcal{A}_{\theta}$. The inverse map is given by

$$
\Omega^{-1}\left(\sum_{(m, r) \in \mathbb{Z}^{2}} a_{m, r} U^{m} V^{r}\right)=\sum_{(m, r) \in \mathbb{Z}^{2}} a_{m, r} \mathrm{e}^{-\pi \mathrm{i} m r \theta} \mathrm{e}^{2 \pi \mathrm{i}(m x+r y)} .
$$

Clearly, the map $\Omega: C^{\infty}\left(\mathbb{T}^{2}\right) \rightarrow \mathcal{A}_{\theta}$ is not an algebra homomorphism; it can be used to deform the commutative product on the algebra $C^{\infty}\left(\mathbb{T}^{2}\right)$ into a noncommutative star-product by defining

$$
f \star g:=\Omega^{-1}(\Omega(f) \Omega(g)), \quad f, g \in C^{\infty}\left(\mathbb{T}^{2}\right) .
$$

A straightforward computation gives

$$
f \star g=\sum_{\left(r_{1}, r_{2}\right) \in \mathbb{Z}^{2}}(f \star g)_{r_{1}, r_{2}} \mathrm{e}^{2 \pi \mathrm{i}\left(r_{1} x+r_{2} y\right)},
$$

with the coefficients of the expansion of the star-product given by a twisted convolution

$$
(f \star g)_{r_{1}, r_{2}}=\sum_{\left(s_{1}, s_{2}\right) \in \mathbb{Z}^{2}} f_{s_{1}, s_{2}} g_{r_{1}-s_{1}, r_{2}-s_{2}} \mathrm{e}^{\pi \mathrm{i}\left(r_{1} s_{2}-r_{2} s_{1}\right) \theta}
$$

which reduces to the usual Fourier convolution product in the limit $\theta=0$. Up to isomorphism, the deformed product depends only on the cohomology class in the group cohomology $\mathrm{H}^{2}\left(\mathbb{Z}^{2}, U(1)\right)$ of the $U(1)$-valued two-cocycle on $\mathbb{Z}^{2}$ given by

$$
\lambda(\boldsymbol{r}, \boldsymbol{s}):=\mathrm{e}^{\pi \mathrm{i}\left(r_{1} s_{2}-r_{2} s_{1}\right) \theta}
$$


with $\boldsymbol{r}=\left(r_{1}, r_{2}\right), s=\left(s_{1}, s_{2}\right) \in \mathbb{Z}^{2}$.

Heuristically, the noncommutative structure (2.3) of the torus is the exponential of the Heisenberg commutation relation $[y, x]=\mathrm{i} \theta / 2 \pi$. Acting on functions of $x$ alone, the operator $U$ is represented as multiplication by $\mathrm{e}^{2 \pi \mathrm{i} x}$ while conjugation by $V$ yields the shift $x \mapsto x+\theta$,

$$
\begin{aligned}
\Omega^{-1}(U \Omega(f(x))) & =\mathrm{e}^{2 \pi \mathrm{i} x} f(x), \\
\Omega^{-1}\left(V \Omega(f(x)) V^{-1}\right) & =f(x+\theta) .
\end{aligned}
$$

Analogously, on functions of $y$ alone we have

$$
\begin{aligned}
\Omega^{-1}\left(U \Omega(g(y)) U^{-1}\right) & =g(y-\theta) \\
\Omega^{-1}(V \Omega(g(y))) & =\mathrm{e}^{2 \pi \mathrm{i} y} g(y) .
\end{aligned}
$$

From (2.3) it follows that $\mathcal{A}_{\theta}$ is commutative if and only if $\theta$ is an integer, and one identifies $\mathcal{A}_{0}$ with the algebra $C^{\infty}\left(\mathbb{T}^{2}\right)$. Also, for any $n \in \mathbb{Z}$ there is an isomorphism $\mathcal{A}_{\theta} \cong \mathcal{A}_{\theta+n}$ since (2.3) does not change under integer shifts $\theta \mapsto \theta+n$. Thus we may restrict the noncommutativity parameter to the interval $0 \leq \theta<1$. Furthermore, since $U V=\mathrm{e}^{-2 \pi \mathrm{i} \theta} V U=\mathrm{e}^{2 \pi \mathrm{i}(1-\theta)} V U$, the correspondence $V \mapsto U, U \mapsto V$ yields an isomorphism $\mathcal{A}_{\theta} \cong \mathcal{A}_{1-\theta}$. These are the only possible isomorphisms and the interval $\theta \in\left[0, \frac{1}{2}\right]$ parametrizes a family of non-isomorphic algebras.

When the deformation parameter $\theta$ is a rational number, the corresponding algebra is related to the commutative torus algebra $C^{\infty}\left(\mathbb{T}^{2}\right)$, i.e. $\mathcal{A}_{\theta}$ is Morita equivalent to it in this case [68]. Let $\theta=p / q$, with $p$ and $q$ integers which we take to be relatively prime with $q>0$. Then $\mathcal{A}_{p / q}$ is isomorphic to the algebra of all smooth sections of an algebra bundle $\mathcal{B}_{p / q} \rightarrow \mathbb{T}^{2}$ whose typical fiber is the algebra $\mathbb{M}_{q}(\mathbb{C})$ of $q \times q$ complex matrices. Moreover, there is a smooth vector bundle $E_{p / q} \rightarrow \mathbb{T}^{2}$ with typical fiber $\mathbb{C}^{q}$ such that $\mathcal{B}_{p / q}$ is the endomorphism bundle $\operatorname{End}\left(E_{p / q}\right)$. With $\omega=\mathrm{e}^{2 \pi \mathrm{i} p / q}$, one introduces the $q \times q$ clock and shift matrices

$$
\mathcal{C}_{q}=\left(\begin{array}{ccccc}
1 & & & & \\
& \omega & & & \\
& \omega^{2} & & \\
& & \ddots & \\
& & & \omega^{q-1}
\end{array}\right) \quad, \quad \mathcal{S}_{q}=\left(\begin{array}{ccccc}
0 & 1 & & & 0 \\
& 0 & 1 & & \\
& & \ddots & \ddots & \\
& & & \ddots & 1 \\
1 & & & & 0
\end{array}\right)
$$


which are unitary and traceless (since $\sum_{k=0}^{q-1} \omega^{k}=0$ ), satisfy

$$
\left(\mathcal{C}_{q}\right)^{q}=\left(\mathcal{S}_{q}\right)^{q}=\mathbb{1}_{q}
$$

and obey the commutation relation

$$
\mathcal{S}_{q} \mathcal{C}_{q}=\omega \mathcal{C}_{q} \mathcal{S}_{q}
$$

Since $p$ and $q$ are relatively prime, the matrices (2.13) generate the finite dimensional algebra $\mathbb{M}_{q}(\mathbb{C})$ : they generate a $C^{*}$-subalgebra which commutes only with multiples of the identity matrix $\mathbb{1}_{q}$, and thus it has to be the full matrix algebra. Were $p$ and $q$ not coprime the generated algebra would be a proper subalgebra of $\mathbb{M}_{q}(\mathbb{C})$. The matrix algebra generated by $\mathcal{C}_{q}$ and $\mathcal{S}_{q}$ is also referred to as the fuzzy torus.

The algebra $\mathcal{A}_{p / q}$ has a "huge" center $\mathcal{C}\left(\mathcal{A}_{p / q}\right)$ which is generated by the elements $U^{q}$ and $V^{q}$, and one identifies $\mathcal{C}\left(\mathcal{A}_{p / q}\right)$ with the commutative algebra $C^{\infty}\left(\mathbb{T}^{2}\right)$ of smooth functions on an ordinary torus $\mathbb{T}^{2}$ which is 'wrapped' $q$ times onto itself. There is a surjective algebra homomorphism

$$
\pi_{q}: \mathcal{A}_{p / q} \longrightarrow \mathbb{M}_{q}(\mathbb{C})
$$

given by

$$
\pi_{q}\left(\sum_{(m, r) \in \mathbb{Z}^{2}} a_{m, r} U^{m} V^{r}\right)=\sum_{(m, r) \in \mathbb{Z}^{2}} a_{m, r}\left(\mathcal{C}_{q}\right)^{m}\left(\mathcal{S}_{q}\right)^{r}
$$

Under this homomorphism the whole center $\mathcal{C}\left(\mathcal{A}_{p / q}\right)$ is mapped to $\mathbb{C}$.

Henceforth we will assume that $\theta$ is an irrational number unless otherwise explicitly stated. On $\mathcal{A}_{\theta}$ there is a unique normalized, positive definite trace which we shall denote by the symbol $f: \mathcal{A}_{\theta} \rightarrow \mathbb{C}$ and which is given by

$$
\begin{aligned}
f \sum_{(m, r) \in \mathbb{Z}^{2}} a_{m, r} U^{m} V^{r} & :=a_{0,0} \\
& =\int_{\mathbb{T}^{2}} \mathrm{~d} x \mathrm{~d} y \Omega^{-1}\left(\sum_{(m, r) \in \mathbb{Z}^{2}} a_{m, r} U^{m} V^{r}\right)(x, y) .
\end{aligned}
$$

Then, for any $a, b \in \mathcal{A}_{\theta}$, one readily checks the properties

$$
f a b=f b a, \quad f \mathbb{1}=1, \quad f a^{\dagger} a>0, a \neq 0
$$


with $f a^{\dagger} a=0$ if and only if $a=0$ (i.e. the trace is faithful). This trace is invariant under the natural action of the commutative torus $\mathbb{T}^{2}$ on $\mathcal{A}_{\theta}$ whose infinitesimal form is determined by two commuting linear derivations $\partial_{1}, \partial_{2}$ acting by

$$
\begin{aligned}
& \partial_{1} U=2 \pi \mathrm{i} U, \quad \partial_{1} V=0, \\
& \partial_{2} U=0, \quad \partial_{2} V=2 \pi \mathrm{i} V .
\end{aligned}
$$

Invariance is just the statement that $f \partial_{\mu} a=0, \mu=1,2$ for any $a \in \mathcal{A}_{\theta}$.

The algebra $\mathcal{A}_{\theta}$ can be represented faithfully as operators acting on a separable Hilbert space $\mathcal{H}$, which is the GNS representation space $\mathcal{H}=$ $L^{2}\left(\mathcal{A}_{\theta}, f\right)$ defined as the completion of $\mathcal{A}_{\theta}$ itself in the Hilbert norm

$$
\|a\|_{\mathrm{GNS}}:=\left(f a^{\dagger} a\right)^{1 / 2}
$$

with $a \in \mathcal{A}_{\theta}$. Since the trace is faithful, the map $\mathcal{A}_{\theta} \ni a \mapsto \widehat{a} \in \mathcal{H}$ is injective and the faithful GNS representation $\pi: \mathcal{A}_{\theta} \rightarrow \mathcal{B}(\mathcal{H})$ is simply given by

$$
\pi(a) \widehat{b}=\widehat{a b}
$$

for any $a, b \in \mathcal{A}_{\theta}$. The vector $1=\widehat{\mathbb{1}}$ of $\mathcal{H}$ is cyclic (i.e. $\pi\left(\mathcal{A}_{\theta}\right) 1$ is dense in $\mathcal{H}$ ) and separating (i.e. $\pi(a) 1=0$ implies $a=0$ ) so that the Tomita involution is just $J(\widehat{a})=\widehat{a^{\dagger}}$ for any $\widehat{a} \in \mathcal{H}$. It is worth mentioning that the $C^{*}$-algebra norm on $\mathcal{A}_{\theta}$ given in (2.2) with $k=0$ coincides with the operator norm in (2.21) when $\mathcal{A}_{\theta}$ is represented on the Hilbert space $\mathcal{H}$, and also with the $L^{\infty}$-norm in the Wigner representation. For ease of notation, in what follows we will not distinguish between elements of the algebra $\mathcal{A}_{\theta}$ and their corresponding operators in the GNS representation.

\subsection{D-Branes as Noncommutative Solitons}

Let us now briefly recall how D-branes arise as soliton configurations which are described as projection operators or partial isometries in the algebra $\mathcal{A}_{\theta}$ of the noncommutative torus ${ }^{1}$. We are interested in systems of unstable D-branes in a closed Type II superstring background of the form $\mathcal{M} \times \mathbb{T}^{2}$. The particular configurations comprise D9-branes in Type IIA string theory and D9- $\overline{\mathrm{D} 9}$ pairs in Type IIB string theory. As it is by now well-known, the effect of turning on a non-degenerate $B$-field along $\mathbb{T}^{2}$ leads to an effective

\footnotetext{
${ }^{1}$ The reader not familiar with, or not interested in, string theory applications may easily skip this subsection without any loss of continuity.
} 
description of the dynamics of these systems in terms of noncommutative geometry [72]. Integrating out all massive string modes in the weakly-coupled open string field theory yields a low-energy effective action that describes a noncommutative field theory of the open string tachyon field $T$ and the open string gauge connection $\nabla$. The classical equations of motion admit interesting soliton solutions $[23,38,78]$ which lead to an open string field theory description of D-branes in terms of tachyon condensation as follows [73].

In the Type IIA case (or alternatively the bosonic string), the tachyon field $T$ on the D9-branes is Hermitian and adjoint-valued, and the tachyon potential is of the form $V\left(T^{2}-\mathbb{1}\right)$, whose global minimum at $T= \pm \mathbb{1}$ is identified as the closed string vacuum containing no perturbative open string excitations. Solving the classical field equations is in general tantamount to seeking slowly-varying tachyonic configurations, i.e. $[\nabla, T]=0$, which extremize the tachyon potential, i.e. $T V^{\prime}\left(T^{2}-\mathbb{1}\right)=0$. One thereby finds solutions in terms of projection operators $\mathrm{P} \in \mathcal{A}_{\theta}$ as

$$
T=\mathbb{1}-\mathrm{P}, \quad \mathrm{P}^{2}=\mathrm{P}=\mathrm{P}^{\dagger} .
$$

A projection operator $\mathrm{P}_{(k)}$ of rank $k$ induces a $U(k)$ gauge symmetry on the lower dimensional unstable D-brane (with worldvolume $\mathcal{M}$ ), whose dynamical degrees of freedom are operators on $\operatorname{ker}(T) \rightarrow \operatorname{ker}(T)$. Since the projections are intimately related to the K-theory of the algebra $\mathcal{A}_{\theta}$, this construction also illustrates the relation between D-branes and K-theory.

In the Type IIB case, the tachyon field on the D9- $\overline{\mathrm{D} 9}$ pairs is complex, and the tachyon potential is of the form

$$
V\left(T, T^{\dagger}\right)=U\left(T^{\dagger} T-\mathbb{1}\right)+U\left(T T^{\dagger}-\mathbb{1}\right),
$$

in order to respect the symmetry given by the action of the operator $(-1)^{F_{\mathrm{L}}}$ which corresponds to interchanging the branes and anti-branes $\left(F_{\mathrm{L}}\right.$ is the left-moving worldsheet fermion number operator). Now the field equations imply that $T$ must satisfy the defining equation of a partial isometry

$$
T T^{\dagger} T=T
$$

The net brane charge is index $(T)$ and, assuming for simplicity that coker $(T)=$ 0 , the dynamical degrees of freedom on the lower-dimensional BPS D-brane again arise from operators on $\operatorname{ker}(T) \rightarrow \operatorname{ker}(T)$.

In both the IIA and IIB situations, the tensions and effective actions of these soliton solutions match exactly with those of the lower dimensional D-branes $[1,37,43]$. In this way the projections and partial isometries of 
$\mathcal{A}_{\theta}$ generate exact D-brane solutions of the equations of motion, with the correct value of the tension. In constructing these D-brane projections, it is convenient to use not just a single projection operator in (2.23), but rather a complete set of mutually orthogonal projections $\mathrm{P}^{i}$ with [43]

$$
\mathrm{P}^{i} \mathrm{P}^{j}=\delta_{i j} \mathrm{P}^{j}, \quad \sum_{i} \mathrm{P}^{i}=\mathbb{1}
$$

Appropriate combinations of the projection operators $\mathrm{P}^{i}$ determine solutions of the Yang-Mills equations on $\mathcal{A}_{\theta}$ [62]. In the following we will construct a natural system of projections and partial isometries which determine matrix regularizations of these sorts of noncommutative field theories.

\subsection{A Sequence of Projections}

The archetype of all projections on the noncommutative two-torus is the Powers-Rieffel projection [68]. To construct it, we first observe that there is an injective algebra homomorphism

$$
\begin{aligned}
\rho: C^{\infty}\left(\mathbb{S}^{1}\right) & \longrightarrow \mathcal{A}_{\theta}, \\
f(x)=\sum_{m \in \mathbb{Z}} f_{m} \mathrm{e}^{2 \pi \mathrm{i} m x} & \longmapsto \rho(f)=\sum_{m \in \mathbb{Z}} f_{m} U^{m}
\end{aligned}
$$

and by using the commutation relations (2.3) it follows, in particular, that if $f(x)$ is mapped to $\rho(f)$, then $V \rho(f) V^{-1}$ is the image of the shifted function $f(x+\theta)$. The map (2.27) is just the Weyl map (2.5) restricted to functions of the variable $x$ alone with the corresponding properties (2.11).

One now looks for projections of the form

$$
\mathrm{P}_{\theta}=V^{-1} \rho(g)+\rho(f)+\rho(g) V
$$

In order that (2.28) define a projection operator, the functions $f, g \in C^{\infty}\left(\mathbb{S}^{1}\right)$ must satisfy some conditions. First of all, they are real-valued and in addition obey

$$
\begin{aligned}
g(x) g(x+\theta) & =0 \\
(f(x)+f(x+\theta)) g(x) & =g(x) \\
g(x)+g(x-\theta) & =\sqrt{f(x)-f(x)^{2}}
\end{aligned}
$$


with $0 \leq f \leq 1$. These conditions are satisfied by putting

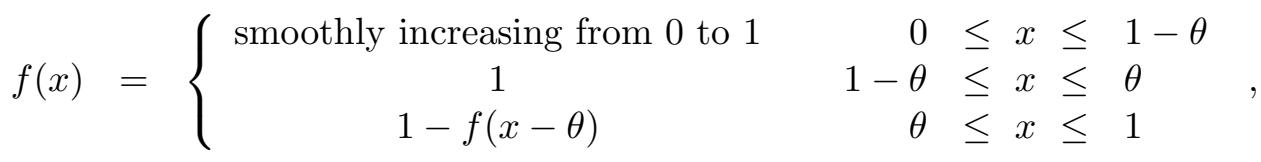

$$
\begin{aligned}
& g(x)=\left\{\begin{array}{cl}
0 & 0 \leq x \leq \theta \\
\sqrt{f(x)-f(x)^{2}} & \theta \leq x \leq 1
\end{array} .\right.
\end{aligned}
$$

There are myriads of other choices possible for these bump functions, and later on we will use a particular one which is useful for our generalizations.

It is straighforward to check that the rank (i.e. trace) of $\mathrm{P}_{\theta}$ is just $\theta$. From (2.28) and the expressions in (2.30) one finds

$$
f \mathrm{P}_{\theta}=f_{0}=\int_{0}^{1} \mathrm{~d} x f(x)=\theta .
$$

Furthermore, the monopole charge (i.e. first Chern number) of $\mathrm{P}_{\theta}$ is 1 . Given any projection $\mathrm{P}$, its Chern number is given by [16]

$$
c_{1}(\mathrm{P})=-\frac{1}{2 \pi \mathrm{i}} f \mathrm{P}\left(\partial_{1} \mathrm{P} \partial_{2} \mathrm{P}-\partial_{2} \mathrm{P} \partial_{1} \mathrm{P}\right) .
$$

This quantity always computes the index of a Fredholm operator, and hence is always an integer. For the projection $\mathrm{P}_{\theta}$ one finds

$$
c_{1}\left(\mathrm{P}_{\theta}\right)=-6 \int_{0}^{1} \mathrm{~d} x g(x)^{2} f^{\prime}(x)=1,
$$

where the last equality follows from the explicit choice (2.30) for the function $f$.

When $\theta$ is an irrational number, together with the trivial projection $\mathbb{1}$, the projection $\mathrm{P}_{\theta}$ generates the $\mathrm{K}_{0}$ group. The trace on $\mathcal{A}_{\theta}$ gives a map

$$
\begin{aligned}
f: \mathrm{K}_{0}\left(\mathcal{A}_{\theta}\right) & \longrightarrow \mathbb{Z}+\mathbb{Z} \theta, \\
r[\mathbb{1}]+m\left[\mathrm{P}_{\theta}\right] & \longmapsto r f \mathbb{1}+m f \mathrm{P}_{\theta}=r+m \theta
\end{aligned}
$$

which is an isomorphism of ordered groups [66]. The positive cone is the collection of (equivalence classes of) projections with positive trace,

$$
\mathrm{K}_{0}^{+}\left(\mathcal{A}_{\theta}\right)=\left\{(r, m) \in \mathbb{Z}^{2} \mid r+m \theta \geq 0\right\} .
$$


The projection $\mathrm{P}_{\theta}$ thereby leads to a complete set of projections for the entire lattice of $\mathrm{D} p-\mathrm{D}(p-2)$ brane charges.

For completeness and later use, let us also add at this point a few remarks about the group $\mathrm{K}_{1}\left(\mathcal{A}_{\theta}\right)$. This group is made up of equivalence classes of homotopic unitary elements in $\mathcal{A}_{\theta}$. It is easy to see that all powers $U^{m} V^{r}$ are mutually non-homotopic. If $U^{m} V^{r}$ and $U^{m^{\prime}} V^{r^{\prime}}$ are homotopic, then so are $\mathrm{e}^{-2 \pi \mathrm{i}\left(m-m^{\prime}\right) r^{\prime} \theta} U^{m-m^{\prime}} V^{r-r^{\prime}}$ and $\mathbb{1}$. But there cannot be a continuous path of unitaries from $U^{m} V^{r}$ to $\mathbb{1}$ since $f U^{m} V^{r}=0$ for $(m, r) \neq(0,0)$, whereas $f \mathbb{1}=1$. Passing to the matrix algebra $\mathbb{M}_{N}\left(\mathcal{A}_{\theta}\right):=\mathcal{A}_{\theta} \otimes \mathbb{M}_{N}(\mathbb{C})$ does not improve the situation since the same argument works with $f$ replaced by $f \otimes \operatorname{Tr}$, where $\operatorname{Tr}$ is the usual $N \times N$ matrix trace. Thus

$$
\mathrm{K}_{1}\left(\mathcal{A}_{\theta}\right)=\mathbb{Z}[U] \oplus \mathbb{Z}[V]
$$

For our purposes we will find it more useful to define two generalized families of projections $\left\{\mathrm{P}_{n}\right\}_{n \geq 1}$ and $\left\{\mathrm{P}_{n}^{\prime}\right\}_{n \geq 1}$ which are related to the even and odd order approximants of the noncommutativity parameter

$$
\theta=\lim _{n \rightarrow \infty} \theta_{n}, \quad \theta_{n}:=\frac{p_{n}}{q_{n}} .
$$

Any irrational number $\theta$ can be treated as a limit (2.37) of rational numbers $\theta_{n}$ in a definite way by using continued fraction expansions. The approximants of $\theta$, as well as the limiting process in (2.37), are described in appendix A, where we also fix some number theoretic notation. For each $n \in \mathbb{N}$, following the Elliott-Evans construction [26], we define two PowersRieffel type projections by

$$
\begin{aligned}
& \mathrm{P}_{n}=V^{-q_{2 n-1}} \rho\left(g_{n}\right)+\rho\left(f_{n}\right)+\rho\left(g_{n}\right) V^{q_{2 n-1}} \\
& \mathrm{P}_{n}^{\prime}=U^{q_{2 n}} \rho^{\prime}\left(g_{n}^{\prime}\right)+\rho^{\prime}\left(f_{n}^{\prime}\right)+\rho^{\prime}\left(g_{n}^{\prime}\right) U^{-q_{2 n}}
\end{aligned}
$$

where $\rho^{\prime}$ is the "dual" of the representation (2.27),

$$
\begin{aligned}
\rho^{\prime}: C^{\infty}\left(\mathbb{S}^{1}\right) & \longrightarrow \mathcal{A}_{\theta}, \\
g(y)=\sum_{r \in \mathbb{Z}} g_{r} \mathrm{e}^{2 \pi \mathrm{i} r y} & \longmapsto \rho^{\prime}(g)=\sum_{r \in \mathbb{Z}} g_{r} V^{r},
\end{aligned}
$$

and now $U \rho^{\prime}(g) U^{-1}$ is the image of the shifted function $g(y-\theta)$. Again, the map (2.40) is just the Weyl map (2.5) restricted to functions of the variable $y$ alone with the corresponding properties (2.12).

The importance of the projections (2.38) and (2.39) is that they provide the building blocks for the construction [26] of a sequence of subalgebras 
$\mathcal{A}_{n} \subset \mathcal{A}_{\theta}$ which converge to the full algebra $\mathcal{A}_{\theta}$ of the noncommutative torus. We shall describe this construction at length in section 3. Each of these subalgebras is a sum of two algebras of matrix-valued functions on a circle. Heuristically, the picture which will emerge is that of two "solitonic fuzzy tori" which wrap around two circles. Any field on the noncommutative torus will thereby admit a regularization by two sets of matrix-valued soliton configurations, each of which is a function on a circle.

In the remainder of this section we will describe the properties of the projections $\mathrm{P}_{n}$ and $\mathrm{P}_{n}^{\prime}$. Since for the time being we will work at a fixed approximation level $n$, to simplify notation we will suppress the subscript $n$ on the functions $f, g, f^{\prime}$ and $g^{\prime}$ and the subscripts $2 n$ and $2 n-1$ on the integers $p$ and $q$. To distinguish $q_{2 n}$ from $q_{2 n-1}$ we will denote the former integer by $q$ and the latter one by $q^{\prime}$, and similarly for $p$. Subscripts will be reintroduced whenever we discuss the limiting process explicitly.

Let us then look for a projection of the form $\mathrm{P}_{n}=V^{-q^{\prime}} \rho(g)+\rho(f)+$ $\rho(g) V^{q^{\prime}}$. As for the Powers-Rieffel projection (2.28), the real-valued functions $f$ and $g$ must now satisfy the conditions

$$
\begin{aligned}
g(x) g\left(x+q^{\prime} \theta\right) & =0 \\
\left(f(x)+f\left(x+q^{\prime} \theta\right)\right) g(x) & =g(x) \\
g(x)+g\left(x-q^{\prime} \theta\right) & =\sqrt{f(x)-f(x)^{2}}
\end{aligned}
$$

with $0 \leq f \leq 1$. We shall also require $f$ to have trace

$$
\beta=p^{\prime}-q^{\prime} \theta
$$

so that $\mathrm{P}_{n}$ is of rank $\beta$, and fix $g$ in such a manner that its $\mathrm{K}_{0}$-class is $\left(p^{\prime},-q^{\prime}\right)$. These numbers $\beta$ also come in a sequence $\left\{\beta_{2 n}\right\}$ which is defined in appendix A, eq. (A.8).

As before, the functions $f$ and $g$ are "bump" functions which now differ from zero only in small intervals. Viewed as continuous functions, they are 
given $b^{2}$

$$
\begin{gathered}
f(x)=\left\{\begin{array}{c}
0 \leq x \leq \frac{1}{2 q}-\delta \\
\frac{1}{\delta-\sigma}\left(x-\frac{1}{2 q}+\delta\right) \\
1 \\
\frac{1}{2 q}-\delta \leq x \leq \frac{1}{2 q}-\sigma \\
\frac{1}{2 q}-\sigma \leq x \leq \frac{1}{2 q}+\sigma \\
\frac{1}{2 q}+\sigma \leq x \leq \frac{1}{2 q}+\delta \\
\frac{1}{2 q}+\delta \leq x \leq 1
\end{array}\right. \\
g(x)=\left\{\begin{array}{cc}
\sqrt{f(x)-f(x)^{2}} & \frac{1}{2 q}-\delta \leq x \leq \frac{1}{2 q}-\sigma \\
0 & \text { otherwise }
\end{array}\right.
\end{gathered}
$$

where $\sigma<\delta<\frac{1}{2 q}$ are positive quantities which are fixed by two conditions. The first one is simply that the trace of $f$ be $\beta=p^{\prime}-q^{\prime} \theta$, i.e. $\int_{0}^{1} \mathrm{~d} x f(x)=\beta$. From the explicit form in (2.43) it is easy to see that the integral is just $\delta+\sigma$. Thus the first condition is

$$
\delta+\sigma=\beta .
$$

The second condition comes from the usage of the projections $\mathrm{P}_{n}$ in the approximation scheme that we mentioned earlier and it ensures the best possible transformation properties for $\mathrm{P}_{n}$ with respect to the generators $U$ and $V[26]$. The condition consists in choosing $f$ to have the least possible slope in the two intervals where it is not constant. The minimal slope is the larger of the two numbers $\beta^{-1}$ and $(1 / q-\beta)^{-1}$ according to whether $\beta$ is smaller or larger than $1 / 2 q$. Again, from the explicit expression in $(2.43)$ the slope is just $(\delta-\sigma)^{-1}$. Thus the second condition is

$$
\delta-\sigma=\left\{\begin{array}{cl}
\beta & \beta \leq \frac{1}{2 q} \\
\frac{1}{q}-\beta & \beta \geq \frac{1}{2 q}
\end{array} .\right.
$$

By putting together the conditions (2.44) and (2.45) we get

$$
\sigma=\left\{\begin{array}{c}
0 \\
\beta-\frac{1}{2 q}
\end{array} \quad, \quad \delta=\left\{\begin{array}{cc}
\beta & \beta \leq \frac{1}{2 q} \\
\frac{1}{2 q} & \beta \geq \frac{1}{2 q}
\end{array} .\right.\right.
$$

Examples of the functions $f$ and $g$ are plotted in Fig. 1 .

One also defines a number $\beta^{\prime}$ by the relations $(1 / q-\beta)^{-1}:=q / q^{\prime} \beta^{\prime}$. This is equivalent to ${ }^{3}$

$$
\beta^{\prime}=q \theta-p,
$$

\footnotetext{
${ }^{2}$ We should really give a "smoothened" version of these bump functions. This can always be accomplished without any difficulty [15] and we will implicitly assume that it has been done whenever necessary.

${ }^{3}$ The sequence $\left\{\beta_{2 n-1}\right\}$ for the $\beta^{\prime}$ 's is defined in appendix A, eq. (A.9).
} 


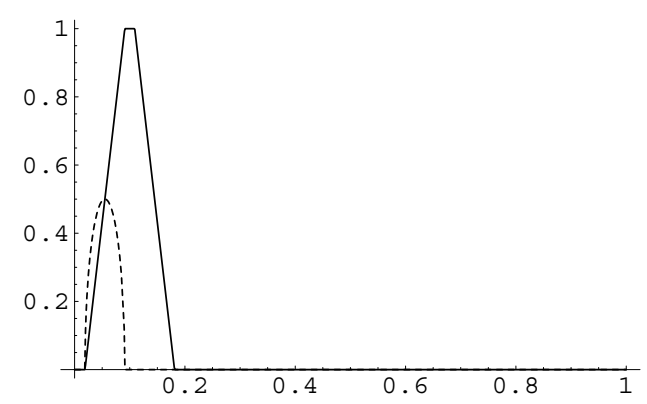

Figure 1: Profiles of the bump functions $f$ (solid line) and $g$ (dashed line) used to construct the projection $\mathrm{P}_{n}$. The noncommutativity parameter is taken to be the inverse of the golden mean, $\theta=\frac{2}{\sqrt{5}+1}$, while the approximants are chosen as $\theta_{2 n}=\frac{3}{5}$ and $\theta_{2 n-1}=\frac{5}{8}$.

from which we have the relation ${ }^{4}$

$$
q \beta+q^{\prime} \beta^{\prime}=1 .
$$

The number $\beta^{\prime}$ plays the same role for the projection $\mathrm{P}_{n}^{\prime}$ as $\beta$ does for $\mathrm{P}_{n}$.

By construction, the rank of $\mathrm{P}_{n}$ is $\beta$,

$$
f \mathrm{P}_{n}=f_{0}=\int_{0}^{1} \mathrm{~d} x f(x)=\beta=p^{\prime}-q^{\prime} \theta,
$$

while its monopole charge is $-q^{\prime}$,

$$
c_{1}\left(\mathrm{P}_{n}\right)=-6 q^{\prime} \int_{0}^{1} \mathrm{~d} x g(x)^{2} f^{\prime}(x)=-q^{\prime},
$$

where the last equality follows from the explicit choice (2.43) for the bump functions. In a completely analogous manner one finds

$$
f \mathrm{P}_{n}^{\prime}=\beta^{\prime}=-p+q \theta, \quad c_{1}\left(\mathrm{P}_{n}^{\prime}\right)=q
$$

Thus the projection $\mathrm{P}_{n}$ in (2.38) represents a soliton configuration carrying brane charges $\left(p_{2 n-1},-q_{2 n-1}\right)$, and the integers $p_{2 n-1}$ and $q_{2 n-1}$ thereby parametrize the vacua of the open string field theory. The rank $\beta$ of $P_{n}$ is

\footnotetext{
${ }^{4}$ See appendix A, eq. (A.10).
} 
the D-brane charge after tachyon condensation. Analogously, the projection $\mathrm{P}_{n}^{\prime}$ has brane charges $\left(-p_{2 n}, q_{2 n}\right)$.

Because these solitons will converge to generic fields on the noncommutative torus, it is instructive to examine their spacetime dependence as elements of $C^{\infty}\left(\mathbb{T}^{2}\right)$. From (2.3), (2.6) and (2.39) we can easily compute the Wigner function on $\mathbb{T}^{2}$ corresponding to the projection $\mathrm{P}_{n}$ in terms of the bump functions (2.43) as

$$
\Omega^{-1}\left(\mathrm{P}_{n}\right)(x, y)=f(x)+2 \cos \left(2 \pi q^{\prime} y\right) g\left(x-\frac{1}{2} q^{\prime} \theta\right)
$$

The soliton field (2.52) represents a typical unstable D7-brane projection configuration and its shape is plotted in Fig. 2. Note that each physical field configuration (2.52) is concentrated in two regions, each of which is localized along the $x$-cycle of the torus but extended along the $y$-direction. It therefore defines tachyonic lumps that have strip-like configurations, unlike the standard point-like configurations of GMS solitons on the noncommutative plane. The first lump has a smooth locus of points and strip area $2 \sigma$ depending on both the D-brane charge and the monopole charge. The second lump contains a periodically spiked locus of support points, with period $q^{\prime}$ and area $\delta-\sigma$. The spiking exemplifies the UV/IR mixing property that generic noncommutative fields possess, in that the size of the configuration decreases as its oscillation period (the monopole charge) grows. Similar considerations can be made for the Wigner function $\Omega^{-1}\left(\mathrm{P}_{n}^{\prime}\right)(x, y)$.

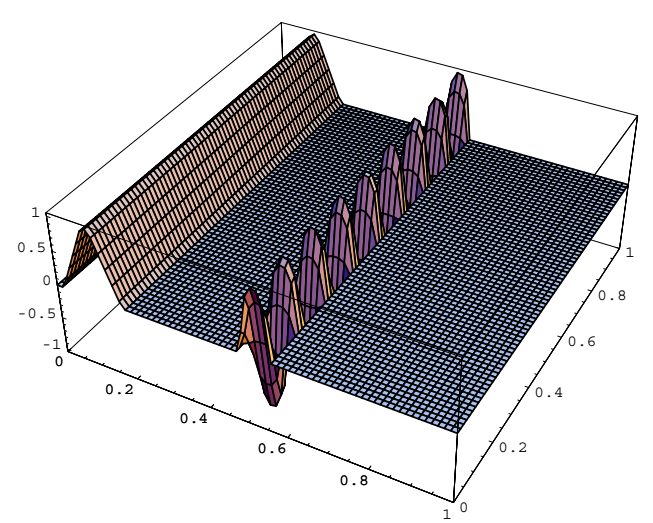

Figure 2: The soliton field configuration corresponding to the projection operator $\mathrm{P}_{n}$ on the noncommutative torus. The noncommutativity parameter is as in Fig. 1. The vertical axis is the Wigner function $\Omega^{-1}\left(\mathrm{P}_{n}\right)(x, y)$ and the horizontal plane is the $(x, y)$-plane. 


\section{Soliton Regularization of Noncommutative Fields}

We will now give the construction of the subalgebras $\mathcal{A}_{n}$ and describe in precisely what sense these subalgebras approximate the full algebra $\mathcal{A}_{\theta}$ of the noncommutative torus [26]. We will also describe how to appropriately truncate fields to $\mathcal{A}_{n}$ in such a manner that they are recovered in the limit $n \rightarrow \infty$. Throughout we shall keep in mind the physical interpretations of these objects within the noncommutative D-brane soliton picture. In this section we shall describe in some detail how the pertinent matrix algebras emerge.

\subsection{From Solitons to Matrix Subalgebras}

For a fixed integer $n$, the subalgebra $\mathcal{A}_{n}$ is constructed starting from the projections $\mathrm{P}_{n}$ and $\mathrm{P}_{n}^{\prime}$ given in (2.38) and (2.39). These two projections will give rise to two towers in $\mathcal{A}_{\theta}$ in which the two unitary generators $U$ and $V$ are treated symmetrically: one of them is modelled in one tower and the second in the other tower. A tower in $\mathcal{A}_{\theta}$ of height $n$ is a family of $n$ orthogonal projections in $\mathcal{A}_{\theta}$ all obtained from a single one by the canonical action of a cyclic subgroup of $\mathbb{S}^{1}=\mathbb{T}^{1}$ of order $n$. In the present case the first tower will be of height $q$, with $q$ projections of trace $\beta=p^{\prime}-q^{\prime} \theta$, while the second tower will be of height $q^{\prime}$, with $q^{\prime}$ projections of trace $\beta^{\prime}=q \theta-p$. The two towers will be orthogonal, i.e. the sum of the projections making up the first tower is the orthogonal complement of the sum of the projections making up the second tower. In order to achieve this it is necessary to adjust the second tower using the fact that any two projections in $\mathcal{A}_{\theta}$ with the same $\mathrm{K}_{0}$-class are unitarily equivalent [69]. From the orthogonality property we must then have that

$$
q\left(p^{\prime}-q^{\prime} \theta\right)+q^{\prime}(q \theta-p)=q p^{\prime}-q^{\prime} p=1
$$

which is just the relation (2.48) (see also (A.10)).

For the rest of this subsection we shall simply write $\mathrm{P}=\mathrm{P}_{n}$ and $\mathrm{P}^{\prime}=\mathrm{P}_{n}^{\prime}$. Given the projection $\mathrm{P}$, we first "translate" it by the (outer) automorphism $\alpha: \mathcal{A}_{\theta} \rightarrow \mathcal{A}_{\theta}$ defined by

$$
\alpha(U)=\mathrm{e}^{2 \pi \mathrm{i} p / q} U, \quad \alpha(V)=V .
$$

The corresponding Wigner function (2.52) is translated accordingly along the $x$-cycle of the torus $\mathbb{T}^{2}$,

$$
\Omega^{-1}(\alpha(\mathrm{P}))(x, y)=\Omega^{-1}(\mathrm{P})(x+p / q, y) .
$$


By repeatedly applying $\alpha$ we can define new projections

$$
\begin{aligned}
\mathrm{P}^{i i}:= & \alpha^{i-1}(\mathrm{P}) \\
= & V^{-q^{\prime}} \rho(g(x+(i-1) p / q)) \\
& +\rho(f(x+(i-1) p / q))+\rho(g(x+(i-1) p / q)) V^{q^{\prime}}
\end{aligned}
$$

for $i=1, \ldots, q$. Since $\alpha^{q}=\mathrm{id}$, it follows that $\mathrm{P}=\mathrm{P}^{11}=\mathrm{P}^{q+1, q+1}$. Moreover, using the explicit form of (2.43) it is straightforward to check that the elements (3.4) form a system of mutually orthogonal projection operators, i.e. $\mathrm{P}^{i i} \mathrm{P}^{j j}=\delta_{i j} \mathrm{P}^{j j}$. As the notation suggests, these projections are the diagonal elements of a basis for a certain matrix subalgebra of $\mathcal{A}_{\theta}$ which we are now going to describe.

Let $\mathcal{H}_{i} \subset \mathcal{H}=L^{2}\left(\mathcal{A}_{\theta}, f\right)$ be the range of the projection $\mathrm{P}^{i i}$. Physically, if $\mathrm{P}^{i i}$ describes a collection of noncommutative D-brane solitons, then $\mathcal{H}_{i}$ is the corresponding Chan-Paton space of the brane configuration, and $\mathrm{P}^{i i} \mathcal{A}_{\theta} \mathrm{P}^{i i}$ is the algebra of endomorphims of this Chan-Paton space. Of course, this space (and its endomorphism algebra) need not be finite-dimensional, in which case the induced D-brane worldvolume carries a $U(\infty)$ gauge symmetry after tachyon condensation owing to the infinite collection of image branes on the torus. This infinite-dimensional symmetry corresponds to invariance of the noncommutative field theory under symplectomorphisms of the D-brane worldvolume [51]. On each of the $\mathcal{H}_{i}$ the corresponding projection $\mathrm{P}^{i i}$ acts as the identity $\mathbb{1}$, while for $j \neq i$ one has $\mathcal{H}_{i} \subset \operatorname{ker}\left(\mathrm{P}^{j j}\right)$. In the D-brane picture, this means that the dynamical degrees of freedom on any pair of distinct non-BPS solitons acts on each other's massless open string states.

We will also need another set of operators which map one Chan-Paton subspace into another, as they will be the off-diagonal elements of the matrix algebra basis. For this, we consider the operator

$$
\Pi^{21}:=\mathrm{P}^{22} V \mathrm{P}^{11} \text {. }
$$

This operator is a mapping from $\mathcal{H}_{1}$ to $\mathcal{H}_{2}$, but is not an isometry, i.e. $\left(\Pi^{21}\right)^{\dagger} \Pi^{21} \neq \mathbb{1}$. This fact may be remedied somewhat by introducing a related partial isometry $\mathrm{P}^{21}$, i.e. an operator for which $\left(\mathrm{P}^{21}\right)^{\dagger} \mathrm{P}^{21}$ and $\mathrm{P}^{21}\left(\mathrm{P}^{21}\right)^{\dagger}$ are projection operators, or equivalently $\mathrm{P}^{21}\left(\mathrm{P}^{21}\right)^{\dagger} \mathrm{P}^{21}=\mathrm{P}^{21}$. Such an operator is given by the partial isometry appearing in the polar decomposition

$$
\Pi^{21}:=\mathrm{P}^{21}\left|\Pi^{21}\right|, \quad\left|\Pi^{21}\right|=\sqrt{\left(\Pi^{21}\right)^{\dagger} \Pi^{21}},
$$

which is well-defined since the operator (3.5) is bounded. The decomposition (3.6) is understood as an equation in the representation of the algebra $\mathcal{A}_{\theta}$ on 
the Hilbert space $\mathcal{H}$, so that $\mathrm{P}^{21} \in \mathcal{A}_{\theta}$. The physical significance of such an operator is that it is unitary in the orthogonal complement to a kernel and a cokernel, and hence will produce localized solitons (in the Wigner representation). The operator $\Pi_{n}^{21}$ and the partial isometry $\mathrm{P}_{n}^{21}$ come arbitrarily close to each other in the large $n$ limit [26], in the sense that

$$
\lim _{n \rightarrow \infty}\left\|\Pi_{n}^{21}-\mathrm{P}_{n}^{21}\right\|_{0}=0 \text {. }
$$

By using (2.3), (2.6) and (3.4), a straightforward calculation gives the Wigner function on $\mathbb{T}^{2}$ corresponding to the operator (3.5) in terms of the periodic bump functions (2.43) as

$$
\begin{aligned}
\mathrm{e}^{-2 \pi \mathrm{i} y} & \Omega^{-1}\left(\Pi^{21}\right)(x, y) \\
= & f\left(x+\frac{p}{q}-\frac{\theta}{2}\right) f\left(x+\frac{\theta}{2}\right)+g\left(x+\frac{p}{q}-\frac{\theta}{2}\right) g\left(x+\frac{\theta}{2}\right) \\
& +g\left(x+\frac{p}{q}-\frac{\left(2 q^{\prime}+1\right) \theta}{2}\right) g\left(x+\frac{\left(2 q^{\prime}-1\right) \theta}{2}\right) \\
& +\mathrm{e}^{4 \pi \mathrm{i} q^{\prime} y} g\left(x+\frac{p}{q}-\frac{\left(2 q^{\prime}+1\right) \theta}{2}\right) g\left(x+\frac{\theta}{2}\right) \\
& +\mathrm{e}^{-4 \pi \mathrm{i} q^{\prime} y} g\left(x+\frac{p}{q}-\frac{\theta}{2}\right) g\left(x-\frac{\left(q^{\prime}-1\right) \theta}{2}\right) \\
& +\mathrm{e}^{2 \pi \mathrm{i} q^{\prime} y}\left[f\left(x+\frac{p}{q}-\frac{\left(q^{\prime}+1\right) \theta}{2}\right) g\left(x-\frac{\left(q^{\prime}-1\right) \theta}{2}\right)\right. \\
& \left.+f\left(x+\frac{\left(q^{\prime}+1\right) \theta}{2}\right) g\left(x+\frac{p}{q}-\frac{\left(q^{\prime}+1\right) \theta}{2}\right)\right] \\
& +\mathrm{e}^{-2 \pi \mathrm{i} q^{\prime} y}\left[f\left(x+\frac{p}{q}+\frac{\left(q^{\prime}-1\right) \theta}{2}\right) g\left(x-\frac{\left(q^{\prime}-1\right) \theta}{2}\right)\right. \\
& \left.+f\left(x-\frac{\left(q^{\prime}-1\right) \theta}{2}\right) g\left(x+\frac{p}{q}-\frac{\left(q^{\prime}+1\right) \theta}{2}\right)\right] .
\end{aligned}
$$

According to (3.7), the function (3.8) represents the typical stable D7-brane soliton partial isometry (at least for sufficiently large approximation level $n$ ). Its shape is plotted in Fig. 3. Again, the multi-soliton image is apparent, with smooth and periodically spiked support loci. Note that while the modulus of the function expectedly displays the characteristic strips of projection solitons, the lumps of its real and imaginary parts are point-like configurations.

Using (3.5) and (3.6), we may now define translated partial isometries analogously to what we did in (3.4) as

$$
\mathrm{P}^{i+2, i+1}:=\alpha^{i}\left(\mathrm{P}^{21}\right), \quad i=1, \ldots, q-2,
$$

where $\alpha$ is the automorphism defined in (3.2). Finally, we also define

$$
\mathrm{P}^{j i}:=\left(\mathrm{P}^{i j}\right)^{\dagger}
$$



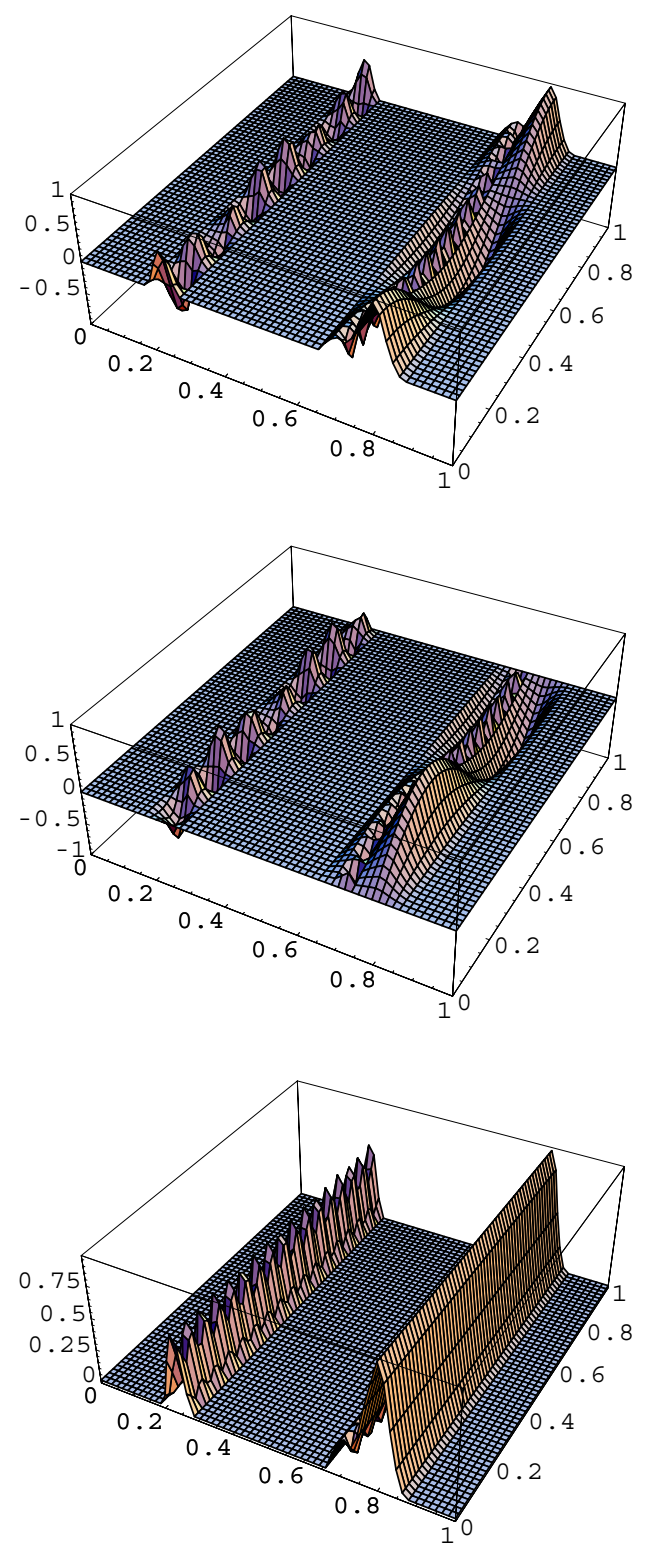

Figure 3: The soliton field configuration corresponding to the operator $\Pi_{n}^{21}$ on the noncommutative torus. Displayed are its real part (top), imaginary part (middle), and modulus (bottom). Parameter values and axes are as in Fig. 2. 
The important fact, proven in appendix B, is that for the operators (3.4) and (3.9) which we have defined, there is a set of relations

$$
\mathrm{P}^{i j} \mathrm{P}^{k l}=\delta_{j k} \mathrm{P}^{i l}
$$

These relations suggest the definition of $q^{2}$ operators $\mathrm{P}^{i j}, 1 \leq i, j \leq q$. The remaining cases $(j \neq i$ and $j \neq i \pm 1)$ are defined by (3.11). For example, $\mathrm{P}^{13}:=\mathrm{P}^{12} \mathrm{P}^{23}$, and so on. Recall that $\mathcal{H}_{i} \subset \mathcal{H}=L^{2}\left(\mathcal{A}_{\theta}, f\right)$ is the range of the projection $\mathrm{P}^{i i}$. Then, the newly defined operators $\mathrm{P}^{i j}$ obtained in this way are partial isometries which are mappings from $\mathcal{H}_{j}$ to $\mathcal{H}_{i}$, i.e. elements of $\mathrm{P}^{i i} \mathcal{A}_{\theta} \mathrm{P}^{j j}$. For the collection of all of them $\left\{\mathrm{P}^{i j}\right\}_{1 \leq i, j \leq q}$, the relation (3.11) holds. In this way we can complete the sets of operators (3.4) and (3.9) into a system of matrix units which generate a $q \times q$ matrix algebra. Because of (3.11), a generic element of this algebra is a complex linear combination $\sum_{i, j} a_{i j} \mathrm{P}^{i j}$ and the product is the usual matrix multiplication.

There is, however, a caveat. The operators $\mathrm{P}^{i+2, i+1}$ in (3.9) are only defined for $i \leq q-2$, and this is in fact sufficient to define all of the $\mathrm{P}^{i j}$ using (3.11), including

$$
\mathrm{P}^{1 q}:=\mathrm{P}^{12} \mathrm{P}^{23} \cdots \mathrm{P}^{q-1, q}
$$

On the other hand, we can also define

$$
\tilde{\mathrm{P}}^{1 q}:=\alpha^{q-1}\left(\mathrm{P}^{21}\right)
$$

For the $q \times q$ matrix algebra to close, it would be necessary that the two operators defined by (3.12) and (3.13) coincide. This is not the case. However, although they are not identical, both of these operators are isometries from $\mathcal{H}_{q}$ to $\mathcal{H}_{1}$. As a consequence, they are related by an operator $z$ which is unitary on $\mathcal{H}_{1}$, i.e. a unitary element of $\mathrm{P}^{11} \mathcal{A}_{\theta} \mathrm{P}^{11}$, and which is therefore a partial isometry on the full Hilbert space $\mathcal{H}$. We therefore have

$$
\tilde{\mathrm{P}}^{1 q}:=z \mathrm{P}^{1 q}
$$

This means that the matrix units $\mathrm{P}^{i j}$, along with the partial isometry $z$, close a subalgebra of $\mathcal{A}_{\theta}$, in which, using (3.11), a generic element is a complex linear combination of the form

$$
\mathrm{A}(z)=\sum_{k \in \mathbb{Z}} \sum_{i, j=1}^{q} a_{i j ; k} z^{k} \mathrm{P}^{i j}
$$

By regarding $z$ as the unitary generator of a circle $\mathbb{S}^{1}$, this subalgebra is (naturally isomorphic to) the algebra $\mathbb{M}_{q}\left(C^{\infty}\left(\mathbb{S}^{1}\right)\right.$ ) of $q \times q$ matrix-valued 
functions on the circle. Since we are interested in continuous and differentiable functions, we will always assume that the complex expansion coefficients $a_{i j ; k}$ in (3.15) are of sufficiently rapid descent as $k \rightarrow \infty$, i.e. $\left\{a_{i j ; k}\right\} \in \mathbb{M}_{q}(\mathbb{C}) \otimes S(\mathbb{Z})$. The identity element of this subalgebra is

$$
\mathbb{1}_{q}=\sum_{i=1}^{q} \mathrm{P}^{i i} .
$$

From the above definitions it follows that the trace of the matrix units is given by

$$
f \mathrm{P}^{i j}=\beta \delta_{i j}
$$

In particular, the identity element (3.16) has trace $f \mathbb{1}_{q}=q \beta$.

In the same way, by starting from the projection $\mathrm{P}^{\prime}=\mathrm{P}_{n}^{\prime}$ in (2.39), a second set of dual projections $\left\{\hat{\mathrm{P}}^{\prime i^{\prime} i^{\prime}}\right\}_{1 \leq i^{\prime} \leq q^{\prime}}$ can be built. This is tantamount to using the $\mathbb{Z}_{4}$ Fourier transformation $U \mapsto V, V \mapsto U^{-1}$ and $(p, q) \leftrightarrow\left(-p^{\prime},-q^{\prime}\right)$ in the above construction. The dual set of projections is not orthogonal in $\mathcal{A}_{\theta}$ to the first set above. However, because of (3.17) and the Diophantine property of appendix A, eq. (A.10), the second set is complementary to the first in the sense that the $\mathrm{K}_{0}$-class of $\sum_{i} \mathrm{P}^{i i}+\sum_{i^{\prime}} \hat{\mathrm{P}}^{\prime i^{\prime} i^{\prime}}$ is equal to the class $(1,0)$ of the unit element of $\mathcal{A}_{\theta}$. It follows that $\sum_{i^{\prime}} \hat{\mathrm{P}}^{\prime i^{\prime} i^{\prime}}$ is unitarily equivalent to $\mathbb{1}-\sum_{i} \mathrm{P}^{i i}$ (as we have mentioned, any two projections in $\mathcal{A}_{\theta}$ with the same $\mathrm{K}_{0}$-class are unitarily equivalent [69]).

We can therefore "rotate" the dual set of projections by conjugating it with the corresponding unitary operator $w$, and thereby obtain a gauge equivalent set of projections which is orthogonal to the first set. This unitary operator can be chosen in such a manner that $\lim _{n \rightarrow \infty}\left\|\left[U, w_{n}\right]\right\|_{0}=$ $\lim _{n \rightarrow \infty}\left\|\left[V, w_{n}\right]\right\|_{0}=0$. This is essential to ensure that the orthogonal direct sum of the two algebras built from each set of projections contains elements approximating the unitary generators $U$ and $V$ of the noncommutative torus $\mathcal{A}_{\theta}$, as will be analysed in more detail in the next subsection. With the gauge transformed dual projections $\mathrm{P}^{\prime i^{\prime} i^{\prime}}:=w \hat{\mathrm{P}}^{\prime i^{\prime} i^{\prime}} w^{\dagger}$, we can now build another set of matrix units $\mathrm{P}^{\prime i^{\prime} j^{\prime}}, 1 \leq i^{\prime}, j^{\prime} \leq q^{\prime}$ which again close a $q^{\prime} \times q^{\prime}$ matrix algebra up to a partial isometry $z^{\prime}$.

By proceeding as before, for each integer $n$, one generates an algebra which is isomorphic to a matrix algebra

$$
\mathcal{A}_{n} \cong \mathbb{M}_{q_{2 n}}\left(C^{\infty}\left(\mathbb{S}^{1}\right)\right) \oplus \mathbb{M}_{q_{2 n-1}}\left(C^{\infty}\left(\mathbb{S}^{1}\right)\right)
$$

The direct sum arises from the orthogonality of the two towers based on the projections $\mathrm{P}_{n}$ and $\mathrm{P}_{n}^{\prime}$, respectively. As we will discuss further later on, 
it is essential to have two copies of such matrix algebras as in (3.18), for $\mathrm{K}$-theoretic reasons. In what follows we will often use a matrix notation for the elements of $\mathcal{A}_{n}$. The matrix elements are always understood to be multiplied by the operators $\mathrm{P}^{i j}$ and $\mathrm{P}^{\prime i^{\prime} j^{\prime}}$ when regarding them as elements of $\mathcal{A}_{\theta}$.

\subsection{The Approximation}

We are now ready to describe in which precise sense the algebra $\mathcal{A}_{n}$ in (3.18) approximates the full noncommutative torus $\mathcal{A}_{\theta}$ [26]. A derivation of this approximation by means of Morita-Rieffel equivalence bimodules is presented in [27] (see also [15]). We stress that $\mathcal{A}_{n}$, being constructed out of elements of the noncommutative torus, is a subalgebra of $\mathcal{A}_{\theta}$. The fact that this subalgebra approximates the noncommutative torus resides in the fact that for each element $a \in \mathcal{A}_{\theta}$, it is possible to construct a corresponding element $\mathrm{a}_{n} \in \mathcal{A}_{n}$ which approximates it in norm. The key ingredients in the construction are two unitary elements $\mathrm{U}_{n}, \mathrm{~V}_{n} \in \mathcal{A}_{n}$ which closely approximate the generators $U$ and $V$ of $\mathcal{A}_{\theta}$. The approximation improves as $n \rightarrow \infty$, whereby the distance, in norm, between $\mathrm{U}_{n}$ and $U$ and between $\mathrm{V}_{n}$ and $V$ becomes arbitrarily small. We will give the matrix expressions for $\mathrm{U}_{n}$ and $\mathrm{V}_{n}$ and the estimate of their difference from $U$ and $V$ without proof, referring to [26] for details. In this subsection we shall reintroduce the subscripts $n$ on all quantities in order to be able to take limits.

As we recall in appendix $\mathrm{A}$, one can approximate the noncommutativity parameter $\theta$ by sequences of even and odd order approximants $\theta_{2 n}<\theta<$ $\theta_{2 n-1}$ with $\theta_{k}=p_{k} / q_{k}$. For each level $n$ we introduce roots of unity

$$
\omega_{n}=\mathrm{e}^{2 \pi \mathrm{i} \theta_{2 n}}, \quad \omega_{n}^{\prime}=\mathrm{e}^{2 \pi \mathrm{i} \theta_{2 n-1}},
$$

with $\left(\omega_{n}\right)^{q_{2 n}}=1=\left(\omega_{n}^{\prime}\right)^{q_{2 n-1}}$. Define

$$
\begin{aligned}
& \mathrm{U}_{n}=\left(\sum_{i=1}^{q_{2 n}}\left(\omega_{n}\right)^{i-1} \mathrm{P}_{n}^{i i}\right) \oplus\left(\sum_{i^{\prime}=1}^{q_{2 n-1}-1} \mathrm{P}_{n}^{\prime i^{\prime}, i^{\prime}+1}+z^{\prime} \mathrm{P}_{n}^{\prime q_{2 n-1}, 1}\right) \\
& =\left(\begin{array}{cc}
\mathcal{C}_{q_{2 n}} & (0)_{q_{2 n} \times q_{2 n-1}} \\
(0)_{q_{2 n-1} \times q_{2 n}} & \mathcal{S}_{q_{2 n-1}}\left(z^{\prime}\right)
\end{array}\right), \\
& \mathrm{V}_{n}=\left(\sum_{i=1}^{q_{2 n}-1} \mathrm{P}_{n}^{i, i+1}+z \mathrm{P}_{n}^{q_{2 n}, 1}\right) \oplus\left(\sum_{i^{\prime}=1}^{q_{2 n-1}}\left(\omega_{n}^{\prime}\right)^{i^{\prime}-1} \mathrm{P}_{n}^{\prime i^{\prime} i^{\prime}}\right) \\
& =\left(\begin{array}{cc}
\mathcal{S}_{q_{2 n}}(z) & (0)_{q_{2 n} \times q_{2 n-1}} \\
(0)_{q_{2 n-1} \times q_{2 n}} & \mathcal{C}_{q_{2 n-1}}
\end{array}\right),
\end{aligned}
$$


where generally $(a)_{q \times r}$ denotes the $q \times r$ matrix whose entries are all equal to $a \in \mathbb{C}$. In these expressions, for any pair of relatively prime integers $p, q$ with $q>0, \mathcal{C}_{q}$ is the $q \times q$ unitary clock matrix as in (2.13), while for any $z \in \mathbb{S}^{1}, \mathcal{S}_{q}(z)$ is the generalized $q \times q$ unitary shift matrix

$$
\mathcal{S}_{q}(z)=\left(\begin{array}{ccccc}
0 & 1 & & & 0 \\
& 0 & 1 & & \\
& & \ddots & \ddots & \\
& & & \ddots & 1 \\
z & & & & 0
\end{array}\right)
$$

with

$$
\left(\mathcal{S}_{q}(z)\right)^{q}=z \mathbb{1}_{q}
$$

The generalization in the shift matrix (3.21) resides in the presence of the generic circular coordinate $z$. It becomes the usual shift matrix in (2.13) when $z$ is taken to be equal to $1, \mathcal{S}_{q}=\mathcal{S}_{q}(1)$.

As mentioned in section 2.1, the clock and shift matrices $\mathcal{C}_{q}$ and $\mathcal{S}_{q}(1)$ form a basis for the finite-dimensional algebra $\mathbb{M}_{q}(\mathbb{C})$ of $q \times q$ complexvalued matrices. By considering both $z$ and $z^{\prime}$ to be the unitary generators of two distinct copies of the algebra $C^{\infty}\left(\mathbb{S}^{1}\right)$, the matrices (3.20) generate the infinite-dimensional algebra $\mathcal{A}_{n} \cong \mathbb{M}_{q_{2 n}}\left(C^{\infty}\left(\mathbb{S}^{1}\right)\right) \oplus \mathbb{M}_{q_{2 n-1}}\left(C^{\infty}\left(\mathbb{S}^{1}\right)\right)$ of matrix-valued functions on two circles. From their definition in $(3.20)$, one finds that

$$
\begin{aligned}
\left(\mathrm{U}_{n}\right)^{q_{2 n} q_{2 n-1}} & =\left(\begin{array}{cc}
\mathbb{1}_{q_{2 n}} & (0)_{q_{2 n} \times q_{2 n-1}} \\
(0)_{q_{2 n-1} \times q_{2 n}} & z^{\prime q_{2 n}} \mathbb{1}_{q_{2 n-1}}
\end{array}\right) \\
\left(\mathrm{V}_{n}\right)^{q_{2 n} q_{2 n-1}} & =\left(\begin{array}{cc}
z^{q_{2 n-1}} \mathbb{1}_{q_{2 n}} & (0)_{q_{2 n} \times q_{2 n-1}} \\
(0)_{q_{2 n-1} \times q_{2 n}} & \mathbb{1}_{q_{2 n-1}}
\end{array}\right)
\end{aligned}
$$

and these matrices generate the center $C^{\infty}\left(\mathbb{S}^{1}\right) \oplus C^{\infty}\left(\mathbb{S}^{1}\right)$ of the algebra $\mathcal{A}_{n}$. Moreover, $\mathrm{U}_{n}$ and $\mathrm{V}_{n}$ have a commutation relation which approximates the one (2.3) of $U$ and $V$,

$$
\mathrm{V}_{n} \mathrm{U}_{n}=\omega_{n} \mathrm{U}_{n} \mathrm{~V}_{n}
$$

with

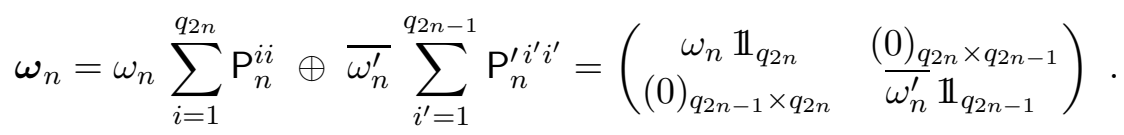

In all of these expressions we have stressed the important double interpretations of these generators. The first equality emphasizes that they are still elements of the algebra $\mathcal{A}_{\theta}$ (i.e. they are expandable in a basis of solitons on 
the noncommutative torus), while the second equality reminds us that they are elements of a matrix algebra (i.e. they are matrix-valued fields on two circles).

Following [26], we will now argue that the matrix algebra $\mathcal{A}_{n}$ "approximates" the noncommutative torus $\mathcal{A}_{\theta}$. Some of the technical details are given in appendix $\mathrm{C}$. As recalled in appendix $\mathrm{A}$, in the limit $n \rightarrow \infty$ both sequences $\theta_{2 n}$ and $\theta_{2 n-1}$ converge to $\theta$ and both sequences $q_{2 n}$ and $q_{2 n-1}$ diverge. Then, the generators $\mathrm{U}_{n}$ and $\mathrm{V}_{n}$ of $\mathcal{A}_{n}$ converge in norm to the generators $U$ and $V$ of $\mathcal{A}_{\theta}$ as

$$
\left\|U-\mathrm{U}_{n}\right\|_{0} \leq \varepsilon_{n}, \quad\left\|V-\mathrm{V}_{n}\right\|_{0} \leq \varepsilon_{n},
$$

where

$$
\varepsilon_{n}=\max \left(\frac{1}{q_{2 n}}, \frac{1}{q_{2 n-1}}\right) C\left(\frac{q_{2 n-1} \beta_{2 n-1}}{q_{2 n} \beta_{2 n}}\right)
$$

and $C$ is a suitable bounded function. For each $n$ one now constructs a projection

$$
\begin{aligned}
\Gamma_{n}: \mathcal{A}_{\theta} & \longrightarrow \mathcal{A}_{n}, \\
\sum_{(m, r) \in \mathbb{Z}^{2}} a_{m, r} U^{m} V^{r} & \longmapsto \Gamma_{n}(a)=\sum_{(m, r) \in \mathbb{Z}^{2}} a_{m, r}\left(\mathrm{U}_{n}\right)^{m}\left(\mathrm{~V}_{n}\right)^{r},
\end{aligned}
$$

which using (3.23) can also be written as

$$
\Gamma_{n}(a)=\sum_{i, j=1}^{q_{2 n} q_{2 n-1}} A_{i j}^{(n)}\left(\mathrm{U}_{n}\right)^{i}\left(\mathrm{~V}_{n}\right)^{j}
$$

where

$$
A_{i j}^{(n)}=\sum_{(m, r) \in \mathbb{Z}^{2}} a_{i+m q_{2 n} q_{2 n-1}, j+r q_{2 n} q_{2 n-1}}\left(\begin{array}{cc}
z^{r q_{2 n-1}} \mathbb{1}_{q_{2 n}} & (0)_{q_{2 n} \times q_{2 n-1}} \\
(0)_{q_{2 n-1} \times q_{2 n}} & z^{\prime m q_{2 n}} \mathbb{1}_{q_{2 n-1}}
\end{array}\right) .
$$

In particular, $\Gamma_{n}(U)=\mathrm{U}_{n}$ and $\Gamma_{n}(V)=\mathrm{V}_{n}$, which along with (3.24) shows that $\Gamma_{n}$ is not an algebra homomorphism. It becomes one, however, in the limit $n \rightarrow \infty$. The crucial fact is that for any element $a \in \mathcal{A}_{\theta}$, its projection $\Gamma_{n}(a)$ is very close to it in norm, in the sense that from (3.26) it follows that their difference goes to zero in the limit,

$$
\lim _{n \rightarrow \infty}\left\|a-\Gamma_{n}(a)\right\|_{0}=0 .
$$

Therefore, to each element of $\mathcal{A}_{\theta}$ there always corresponds an element of the subalgebra $\mathcal{A}_{n}$ to within an arbitrarily small radius. A generic element 
$a \in \mathcal{A}_{\theta}$ can be approximated to arbitrarily good precision by a matrix-valued function on two circles of the form

$$
\Gamma_{n}(a)=\mathrm{a}_{n}\left(z, z^{\prime}\right)=\left(\begin{array}{cc}
\mathrm{a}_{n}(z) & (0)_{q_{2 n} \times q_{2 n-1}} \\
(0)_{q_{2 n-1} \times q_{2 n}} & \mathrm{a}_{n}^{\prime}\left(z^{\prime}\right)
\end{array}\right),
$$

with $\mathrm{a}_{n}(z) \in \mathbb{M}_{q_{2 n}}\left(C^{\infty}\left(\mathbb{S}^{1}\right)\right)$ and $\mathrm{a}_{n}^{\prime}\left(z^{\prime}\right) \in \mathbb{M}_{q_{2 n-1}}\left(C^{\infty}\left(\mathbb{S}^{1}\right)\right)$. Only the information about the higher momentum modes $a_{m, r}$ of the expansion of $a$ is lost (i.e. for $m, r>q_{2 n} q_{2 n-1}$ ), and these coefficients are small for Schwartz sequences. Hence the approximation for large $n$ is good.

It is possible to prove an even stronger result [26] which gives a concrete realization of the noncommutative torus as the inductive limit $\mathcal{A}_{\theta}=\bigcup_{n=0}^{\infty} \mathcal{B}_{n}$ of an appropriate inductive system of algebras $\left\{\mathcal{B}_{n}, \iota_{n}\right\}_{n \geq 0}$, together with injective unital $*$-morphisms $\iota_{n}: \mathcal{B}_{n} \hookrightarrow \mathcal{B}_{n+1}$. It turns out that, for Ktheoretical reasons, the finite level algebras $\mathcal{B}_{n}$ must be taken as $\mathcal{B}_{n}=\mathcal{A}_{2 n+1}$, with the latter algebra of the form (3.18). The crucial issue here is that the embeddings from one algebra to the next one must be taken in such a way that, in the limit, the K-theory groups (2.34) and (2.36) of the noncommutative torus are obtained. That a judicious choice here is indeed possible follows from the $\mathrm{K}$-theoretic properties $\mathrm{K}^{0}\left(\mathbb{S}^{1}\right)=\mathrm{K}^{1}\left(\mathbb{S}^{1}\right)=\mathbb{Z}$ of the circle, so that by Morita equivalence the K-theory groups of the matrix algebras $\mathcal{A}_{n}$ are given by

$$
\mathrm{K}_{0}\left(\mathcal{A}_{n}\right)=\mathrm{K}_{1}\left(\mathcal{A}_{n}\right)=\mathbb{Z} \oplus \mathbb{Z},
$$

with the canonical ordering $\mathrm{K}_{0}^{+}\left(\mathcal{A}_{n}\right)=\mathbb{N}_{0} \oplus \mathbb{N}_{0}$ for the dimension group. The details are described in appendix D. A very heuristic explanation for the necessity of using two towers in the matrix regularization will be given in the next section.

The physical interpretation of the projection (3.29) should be clear. On the original noncommutative torus, there is an infinite number of image D-branes parametrized by the momentum lattice $\mathbb{Z}^{2}$ of the quotient space $\mathbb{T}^{2}=\mathbb{R}^{2} / \mathbb{Z}^{2}$ used to construct the brane configurations from the universal cover of the torus. The mapping (3.29) thereby corresponds to a truncation of fields on the noncommutative torus in such a way that there are only a finite number $q_{2 n} q_{2 n-1}$ of image D-branes on $\mathbb{T}^{2}$ at each level $n$, corresponding to the collection of physical open string modes which are invariant under the action of the cyclic group $\mathbb{Z}_{q_{2 n} q_{2 n-1}} \times \mathbb{Z}_{q_{2 n} q_{2 n-1}}$. The Wigner map can also be used to determine the finite two-cocycle that appears in the twisted convolution of the image of the product in the finite algebra, giving the ana$\log$ of (2.9) for the noncommutative torus, although we shall not investigate this matter here.

Instead, in what follows it will be more useful to encode the noncom- 
mutativity of the algebra $\mathcal{A}_{n}$ by using the usual matrix multiplication of functions on a circle $\mathbb{S}^{1}$. We then obtain an expansion of noncommutative fields in terms of both stable and unstable D-brane solitons on the torus $\mathbb{T}^{2}$. The remarkable fact about this soliton expansion is that it leads to a description of the dynamics of a noncommutative field in a very precise way in terms of a one-dimensional matrix model, whose (inductive) limit reproduces exactly the original continuum dynamics. This is quite unlike the situation with the zero-dimensional matrix model regularizations of noncommutative field theory $[4,46,5]$, whereby the finite-dimensional matrix algebras can never realize the noncommutative torus as an inductive limit [65]. In the present case the regularization in fact mimicks most properties of the continuum field theory already at the finite level, owing to this much stronger limiting behaviour. In the following we shall explore the implications of the soliton regularization within this context.

\section{Noncommutative Field Theory as Matrix Quan- tum Mechanics}

In this section we shall go back to the setting of section 2.2 and consider open superstring field theory on the background $\mathcal{M} \times \mathbb{T}^{2}$. As discussed there, in the presence of a constant $B$-field the tachyon fields $T$ are functions on $\mathcal{M} \rightarrow \mathcal{A}_{\theta}$. The generic situation we will therefore consider is that there is a set of fields, which we denote collectively by $\Phi$, with Lagrangian density $\mathcal{L}$, all of which are functions on $\mathcal{M} \rightarrow \mathcal{A}_{\theta}$. By remembering that on the algebra $\mathcal{A}_{\theta}$ the integration is given by the trace (2.18), the action for noncommutative string field theory compactified on a two-torus can be written schematically as

$$
S=\frac{g_{s} \mu_{9}}{G_{s}} \int_{\mathcal{M}} \sqrt{\operatorname{det} G} f \mathcal{L}\left[\Phi, \partial_{\mu} \Phi\right],
$$

where $G_{s}$ and $G_{\mu \nu}$ are the effective coupling and metric felt by the open strings in the presence of the constant $B$-field along $\mathbb{T}^{2}, g_{s}$ is the closed string coupling constant, and $\mu_{9}$ is the spacetime-filling D-brane tension in the absence of the $B$-field. The derivatives $\partial_{\mu}, \mu=1,2$ are the canonical linear derivations on $\mathcal{A}_{\theta}$ defined in (2.20).

We will now use the mapping (3.28) onto the approximating subalgebra $\mathcal{A}_{n}$ in (3.18) to build a matrix field theory which regulates the field theory (4.1) on $\mathcal{A}_{\theta}$. Using (3.32) we replace the fields $\Phi$ on $\mathcal{A}_{\theta}$ by the fields $\boldsymbol{\Phi}_{n}\left(z, z^{\prime}\right)$ on $\mathcal{A}_{n}$ which are direct sums of $q_{2 n} \times q_{2 n}$ matrix fields $\boldsymbol{\Phi}_{n}(z)$ and $q_{2 n-1} \times q_{2 n-1}$ matrix fields $\boldsymbol{\Phi}_{n}^{\prime}\left(z^{\prime}\right)$ on $\mathbb{S}^{1}$. We need to examine the actions of 
the trace (2.18) and derivative (2.20) on the algebra $\mathcal{A}_{n}$. We will reinterpret them as operations on the matrix algebras $\mathcal{A}_{n}$, without reference to their embeddings as subalgebras of the noncommutative torus $\mathcal{A}_{\theta}$, which in the $n \rightarrow \infty$ limit converge to the trace and derivative on $\mathcal{A}_{\theta}$. The resulting matrix quantum mechanics can be regarded as a non-perturbative regularization of the original continuum field theory on the noncommutative torus, which is obtained as the limit $n \rightarrow \infty$.

\subsection{Spacetime Averages}

Let us start with the canonical trace. On elements of $\mathcal{A}_{\theta}$ the trace (2.18) is determined through the definition $f U^{m} V^{r}:=\delta_{m 0} \delta_{r 0}$. To determine the trace of corresponding elements of $\mathcal{A}_{n}$, we note that because of (3.17), traces of powers $\left(\mathrm{U}_{n}\right)^{m}\left(\mathrm{~V}_{n}\right)^{r}$ of the generators (3.20) vanish unless the corresponding powers of both the clock and shift operators are proportional to the identity elements $\mathbb{1}_{q_{2 n}}$ or $\mathbb{1}_{q_{2 n-1}}$, which happens whenever $m$ and $r$ are arbitrary integer multiples of $q_{2 n}$ or $q_{2 n-1}$. From the definitions of the unitaries $z$ and $z^{\prime}$, we further have

$$
f z^{m} \mathbb{1}_{q_{2 n}}=q_{2 n} \beta_{2 n} \delta_{m 0}, \quad f z^{\prime m} \mathbb{1}_{q_{2 n-1}}=q_{2 n-1} \beta_{2 n-1} \delta_{m 0},
$$

from which it follows that

$$
f\left(\mathrm{U}_{n}\right)^{m}\left(\mathrm{~V}_{n}\right)^{r}=\sum_{k \in \mathbb{Z}}\left(q_{2 n} \beta_{2 n} \delta_{m, q_{2 n} k} \delta_{r 0}+q_{2 n-1} \beta_{2 n-1} \delta_{m 0} \delta_{r, q_{2 n-1} k}\right) .
$$

In the large $n$ limit, by using (A.10) we see that the trace $f \Gamma_{n}(a)$ is therefore well approximated by $a_{0,0}$, since the correction terms $a_{q_{2 n} k, 0}$ and $a_{0, q_{2 n-1} k}$ for $k \neq 0$ are then small for Schwartz sequences. It is now clear how to rewrite $f \Gamma_{n}(a)$ in terms of operations which are intrinsic to the matrix algebras (3.18). The trace (4.2) can be reproduced on functions on $\mathbb{S}^{1}$ by integration over the circle, while the trace of the matrix degrees of freedom are ordinary $q_{2 n} \times q_{2 n}$ and $q_{2 n-1} \times q_{2 n-1}$ matrix traces $\operatorname{Tr}$ and $\operatorname{Tr}^{\prime}$, respectively, accompanied by the appropriate normalizations $q_{2 n} \beta_{2 n}$ and $q_{2 n-1} \beta_{2 n-1}$. In $\mathcal{A}_{n}$, we regard $z$ and $z^{\prime}$ as ordinary circular coordinates and set $z:=\mathrm{e}^{2 \pi \mathrm{i} \tau}$, with $\tau \in[0,1)$, and $z^{\prime}:=\mathrm{e}^{2 \pi \mathrm{i} \tau^{\prime}}$, with $\tau^{\prime} \in[0,1)$. It then follows that the trace of a generic element (3.32) can be written solely in terms of matrix quantities as

$$
f \mathrm{a}_{n}=\beta_{2 n} \int_{0}^{1} \mathrm{~d} \tau \operatorname{Tr} \mathrm{a}_{n}(\tau)+\beta_{2 n-1} \int_{0}^{1} \mathrm{~d} \tau^{\prime} \operatorname{Tr}^{\prime} \mathrm{a}_{n}^{\prime}\left(\tau^{\prime}\right) .
$$




\subsection{Kinetic Energies}

The definition of the equivalent of the derivations (2.20) is somewhat more involved. We will define two approximate derivations $\nabla_{\mu}, \mu=1,2$ on $\mathcal{A}_{n}$, which in the limit $n \rightarrow \infty$ approach the $\partial_{\mu}$ 's. They are approximate derivations in the sense that the Leibniz rule holds only in the limit. They are, however, sufficient for the definition of the regulated action. For this, let us look more closely at the map $a \mapsto \mathrm{a}_{n}=\Gamma_{n}(a)$ defined in $(3.29,3.30)$, and express it as a power series expansion

$$
\begin{aligned}
\mathrm{a}_{n}\left(z, z^{\prime}\right)= & \mathrm{a}_{n}(z) \oplus \mathrm{a}_{n}^{\prime}\left(z^{\prime}\right) \\
= & \sum_{i, j=1}^{q_{2 n}} \sum_{k \in \mathbb{Z}} \alpha_{i+\left[\frac{q_{2 n}}{2}\right]_{0}, j ; k}^{(n)} z^{k}\left(\mathcal{C}_{q_{2 n}}\right)^{i}\left(\mathcal{S}_{q_{2 n}}(z)\right)^{j} \\
& \oplus \sum_{i^{\prime}, j^{\prime}=1}^{q_{2 n-1}} \sum_{k^{\prime} \in \mathbb{Z}} \alpha_{i^{\prime}, j^{\prime}+\left[\frac{q_{2 n-1}}{2}\right]_{0} ; k^{\prime}} z^{\prime k^{\prime}}\left(\mathcal{S}_{q_{2 n-1}}\left(z^{\prime}\right)\right)^{i^{\prime}}\left(\mathcal{C}_{q_{2 n-1}}\right)^{j^{\prime}}
\end{aligned}
$$

where $[\cdot]_{0}$ denotes the integer part. Notice that the roles of the clock and generalized shift matrices are interchanged between the two towers. The shift in the first index of the expansion coefficients $\alpha^{(n)}$ in the first tower effectively sets the range of the powers of the clock operators to lie symmetrically about 0 in the range $-\left[\frac{q_{2 n}}{2}\right]_{0}, \ldots,\left[\frac{q_{2 n}}{2}\right]_{0}$. It is made for technical reasons that will become clearer below. An analogous argument holds for the second index of $\alpha^{\prime(n)}$ in the second tower. The remaining momentum modes lie in the range $j=1, \ldots, q_{2 n}$ and $i^{\prime}=1, \ldots, q_{2 n-1}$. While differences between the various index range conventions vanish in the limit $n \rightarrow \infty$, they do affect the convergence properties of the finite level approximations.

The expansion coefficients of $(4.5)$ may be computed from $(3.29,3.30)$ to get

$$
\begin{aligned}
\alpha_{i, j ; k}^{(n)} & =\sum_{l \in \mathbb{Z}} a_{i+q_{2 n} l-\left[\frac{q_{2 n}}{2}\right]_{0}, j+q_{2 n} k}, \\
\alpha_{i^{\prime}, j^{\prime} ; k^{\prime}}^{\prime(n)} & =\sum_{l^{\prime} \in \mathbb{Z}} a_{i^{\prime}+q_{2 n-1} k^{\prime}, j^{\prime}+q_{2 n-1} l^{\prime}-\left[\frac{q_{2 n-1}}{2}\right]_{0} .} .
\end{aligned}
$$

In the first tower the coefficients of the high momentum modes of $U$ are summed to low momentum ones. However, for Schwartz sequences this "correction" is small and vanishes as $q_{2 n} \rightarrow \infty$. The choice of the range of powers of the clock matrices made in (4.5) was in fact motivated by the necessity to be able to ignore these small coefficients. In the second tower it is the high momentum modes of $V$ which are lost. The interplay between 
the two towers is such that they neglect different high momentum modes, so that in a certain sense the two errors "compensate" each other. The representation $(4.5,4.6)$ thereby provides a nice heuristic insight into the role of the two towers in the matrix regularization.

Let us now look at the projection of $\partial_{1} a=2 \pi \mathrm{i} \sum_{m, r} m a_{m, r} U^{m} V^{r}$ in the two towers. By using (4.6) the corresponding expansion coefficients may be written as

$$
\begin{aligned}
\left(\Gamma_{n}\left(\partial_{1} a\right)\right)_{i, j ; k} & =2 \pi \mathrm{i} \sum_{l \in \mathbb{Z}}\left(i+q_{2 n} l-\left[\frac{q_{2 n}}{2}\right]_{0}\right) a_{i+q_{2 n} l-\left[\frac{q_{2 n}}{2}\right]_{0}, j+q_{2 n} k} \\
& =2 \pi \mathrm{i}\left(i-\left[\frac{q_{2 n}}{2}\right]_{0}\right) \alpha_{i, j ; k}^{(n)}+O\left(q_{2 n} a_{i-\left[\frac{q_{2 n}}{2}\right]_{0}, q_{2 n} k}\right) \\
\left(\Gamma_{n}\left(\partial_{1} a\right)\right)_{i^{\prime}, j^{\prime} ; k^{\prime}}^{\prime} & =2 \pi \mathrm{i} \sum_{l^{\prime} \in \mathbb{Z}}\left(i^{\prime}+q_{2 n-1} k^{\prime}\right) a_{i^{\prime}+q_{2 n-1} k^{\prime}, j^{\prime}+q_{2 n-1} l^{\prime}-\left[\frac{q_{2 n-1}}{2}\right]_{0}} \\
& =2 \pi \mathrm{i}\left(i^{\prime}+q_{2 n-1} k^{\prime}\right) \alpha_{i^{\prime}, j^{\prime} ; k^{\prime}}^{(n)} .
\end{aligned}
$$

In the first set of equalities in (4.7) the neglected terms in the second equality vanish for Schwartz sequences as $n \rightarrow \infty$, while in the second set no approximation is necessary. The same reasoning can be repeated for the projection of $\partial_{2} a$, and together these results suggest the definitions

$$
\begin{aligned}
& \nabla_{1} \mathrm{a}_{n}\left(z, z^{\prime}\right)=2 \pi \mathrm{i}\left[\sum_{i, j=1}^{q_{2 n}} \sum_{k \in \mathbb{Z}} i \alpha_{i+\left[\frac{q_{2 n}}{2}\right]_{0}, j ; k}^{(n)} z^{k}\left(\mathcal{C}_{q_{2 n}}\right)^{i}\left(\mathcal{S}_{q_{2 n}}(z)\right)^{j}\right. \\
& \oplus \sum_{i^{\prime}, j^{\prime}=1}^{q_{2 n-1}} \sum_{k^{\prime} \in \mathbb{Z}}\left(i^{\prime}+q_{2 n-1} k^{\prime}\right) \alpha_{i^{\prime}, j^{\prime}+\left[\frac{q_{2 n-1}}{2}\right]_{0} ; k^{\prime}}^{(n)} z^{\prime k^{\prime}} \\
& \left.\times\left(\mathcal{S}_{q_{2 n-1}}\left(z^{\prime}\right)\right)^{i^{\prime}}\left(\mathcal{C}_{q_{2 n-1}}\right)^{j^{\prime}}\right], \\
& \nabla_{2} \mathrm{a}_{n}\left(z, z^{\prime}\right)=2 \pi \mathrm{i}\left[\sum_{i, j=1}^{q_{2 n}} \sum_{k \in \mathbb{Z}}\left(j+q_{2 n-1} k\right) \alpha_{i+\left[\frac{q_{2 n}}{2}\right]_{0}, j ; k}^{(n)} z^{k}\right. \\
& \times\left(\mathcal{C}_{q_{2 n}}\right)^{i}\left(\mathcal{S}_{q_{2 n}}(z)\right)^{j} \\
& \left.\oplus \sum_{q_{2 n-1}}^{q^{\prime}, j^{\prime}=1} \sum_{k^{\prime} \in \mathbb{Z}} j^{\prime} \alpha_{i^{\prime}, j^{\prime}+\left[\frac{q_{2 n-1}}{2}\right]_{0} ; k^{\prime}} z^{\prime k^{\prime}}\left(\mathcal{S}_{q_{2 n-1}}\left(z^{\prime}\right)\right)^{i^{\prime}}\left(\mathcal{C}_{q_{2 n-1}}\right)^{j^{\prime}}\right] .
\end{aligned}
$$

These two operations converge to the canonical linear derivations on the 
algebra $\mathcal{A}_{\theta}$ and satisfy an approximate Leibniz rule which is proven in appendix E.

We now wish to express the "derivatives" (4.8) as operations acting on $\mathrm{a}_{n}\left(z, z^{\prime}\right)$ expressed as a pair of matrix-valued functions on circles,

$$
\mathrm{a}_{n}\left(z, z^{\prime}\right)=\sum_{k \in \mathbb{Z}} \sum_{i, j=1}^{q_{2 n}} a_{i j ; k}^{(n)} z^{k} \mathrm{P}_{n}^{i j} \oplus \sum_{k^{\prime} \in \mathbb{Z}} \sum_{i^{\prime}, j^{\prime}=1}^{q_{2 n-1}} a_{i^{\prime} j^{\prime} ; k^{\prime}}^{\prime(n)} z^{\prime k^{\prime}} \mathrm{P}_{n}^{\prime i^{\prime} j^{\prime}} .
$$

For this, we need to find the appropriate change of matrix basis between the two expansions (4.5) and (4.9). We will do this explicitly below only for the first tower, the analogous formulæ for the second tower being the obvious modifications.

The key formula which enables this change of basis is provided by the identity

$\left(\mathcal{C}_{q_{2 n}}\right)^{i}\left(\mathcal{S}_{q_{2 n}}(z)\right)^{j}=\sum_{s=1}^{q_{2 n}-j}\left(\omega_{n}\right)^{i(s-1)} \mathrm{P}_{n}^{s, s+j}+z \sum_{s=q_{2 n}-j+1}^{q_{2 n}}\left(\omega_{n}\right)^{i(s-1)} \mathrm{P}_{n}^{s, s+j-q_{2 n}}$,

which is readily derived from the orthonormality relation (3.11). A straightforward consequence of (4.10), the trace formula (3.17), and the identity

$$
\frac{1}{q_{2 n}} \sum_{t=1}^{q_{2 n}}\left(\omega_{n}\right)^{t\left(s-s^{\prime}\right)}=\delta_{s s^{\prime}} \quad \text { for } \quad s, s^{\prime} \in \mathbb{Z}_{q_{2 n}}
$$

is that the elements of the matrix basis of the expansion (4.5) are orthogonal,

$$
\operatorname{Tr}\left[\left(\mathcal{S}_{q_{2 n}}(z)^{\dagger}\right)^{j}\left(\mathcal{C}_{q_{2 n}}^{\dagger}\right)^{i}\right]\left[\left(\mathcal{C}_{q_{2 n}}\right)^{s}\left(\mathcal{S}_{q_{2 n}}(z)\right)^{t}\right]=q_{2 n} \beta_{2 n} \delta_{i s} \delta_{j t} .
$$

From (4.12) it follows that the expansion coefficients of (4.5) may thereby be computed as

$$
\alpha_{i+\left[\frac{q_{2 n}}{2}\right]_{0}, j ; k}^{(n)}=\frac{1}{q_{2 n} \beta_{2 n}} \oint \frac{\mathrm{d} z}{2 \pi \mathrm{i} z^{k+1}} \operatorname{Tr} \mathrm{a}_{n}(z)\left(\mathcal{S}_{q_{2 n}}(z)^{\dagger}\right)^{j}\left(\mathcal{C}_{q_{2 n}}^{\dagger}\right)^{i} .
$$

By substituting (4.9) into (4.13), and using (4.10) along with (3.17), after some algebra we arrive at the change of basis $a_{i j ; k}^{(n)} \mapsto \alpha_{i, j ; k}^{(n)}$ in the form

$$
\begin{aligned}
\alpha_{i+\left[\frac{q_{2 n}}{2}\right]_{0}, j ; k}^{(n)}= & \frac{1}{q_{2 n}}\left[\sum_{s=1}^{q_{2 n}-j} a_{s, s+j ; k}^{(n)}\left(\omega_{n}\right)^{-i(s-1)}\right. \\
& \left.+\sum_{s=q_{2 n}-j+1}^{q_{2 n}} a_{s, s+j-q_{2 n} ; k+1}^{(n)}\left(\omega_{n}\right)^{-i(s-1)}\right] .
\end{aligned}
$$


On the other hand, from (3.17) it follows that the expansion coefficients of (4.9) may be computed from

$$
a_{i j ; k}^{(n)}=\frac{1}{\beta_{2 n}} \oint \frac{\mathrm{d} z}{2 \pi \mathrm{i} z^{k+1}} \operatorname{Tr} \mathrm{a}_{n}(z) \mathrm{P}_{n}^{j i}
$$

By substituting (4.5) into (4.15), and again applying (4.10) and (3.17), we arrive at the change of basis $\alpha_{i, j ; k}^{(n)} \mapsto a_{i j ; k}^{(n)}$ in the form

$$
a_{i j ; k}^{(n)}=\sum_{s=1}^{q_{2 n}}\left(\omega_{n}\right)^{s(i-1)} \times\left\{\begin{array}{cc}
\alpha^{(n)}{ }_{s+\left[\frac{q_{2 n}}{2}\right]_{0}, j-i ; k} & i<j \\
\alpha_{s+\left[\frac{q_{2 n}}{2}\right]_{0}, q_{2 n}+j-i ; k-1}^{(n)} & i \geq j
\end{array}\right.
$$

Let us now deal with the derivative $\nabla_{1}$ acting on the first tower in (4.8), and substitute $i \alpha_{i+\left[\frac{q_{2 n}}{2}\right]_{0}, j ; k}^{(n)}$ in place of $\alpha_{i+\left[\frac{q_{2 n}}{2}\right]_{0}, j ; k}^{(n)}$ in (4.16) to obtain the canonical matrix elements $b_{i, j ; k}^{(n)}$ of the expansion of $\nabla_{1} \mathrm{a}_{n}\left(z, z^{\prime}\right)$ analogous to (4.9). For $i<j$, it follows from (4.14) that they are given by

$$
\begin{aligned}
b_{i, j ; k}^{(n)}= & \frac{1}{q_{2 n}} \sum_{s=1}^{q_{2 n}} s\left(\omega_{n}\right)^{s i}\left[\sum_{s^{\prime}=1}^{q_{2 n}-j+i} a_{s^{\prime}, s^{\prime}+j-i ; k}^{(n)}\left(\omega_{n}\right)^{-s s^{\prime}}\right. \\
& \left.+\sum_{s^{\prime}=q_{2 n}+i-j+1}^{q_{2 n}} a_{s^{\prime}, s^{\prime}+j-i-q_{2 n} ; k+1}^{(n)}\left(\omega_{n}\right)^{-s s^{\prime}}\right],
\end{aligned}
$$

with a similar expression in the case $i \geq j$. To understand the geometrical meaning of the expression (4.17), we recall that the translation generators on the ordinary torus $\mathbb{T}^{2}$ are given by

$$
\mathrm{e}^{-x_{0} \partial_{1}} f(x, y) \mathrm{e}^{x_{0} \partial_{1}}=f\left(x+x_{0}, y\right)
$$

plus the analogous expression for the shift in $y$. The canonical derivations on the fuzzy torus, i.e. the discrete versions of these operators, are realized in a unitary fashion (rather than Hermitian) and are given by clock and shift matrices as $[4,5]$

$$
\begin{aligned}
& \left(\mathcal{C}_{q_{2 n}}^{\dagger} \mathrm{a}_{n} \mathcal{C}_{q_{2 n}}\right)_{i, j ; k}=\left(\omega_{n}\right)^{j-i} a_{i j ; k}^{(n)}, \\
& \left(\mathcal{S}_{q_{2 n}}^{\dagger} \mathrm{a}_{n} \mathcal{S}_{q_{2 n}}\right)_{i j ; k}=a_{[i-1]_{q_{2 n}},[j+1]_{q_{2 n} ; k}}^{(n)}
\end{aligned}
$$

with $[\cdot]_{q}$ denoting the integer part modulo $q$. Given that the periodic deltafunction on the cyclic group $\mathbb{Z}_{q_{2 n}}$ is represented by the finite Fourier transform (4.11) in terms of the $q_{2 n}$-th roots of unity $\omega_{n}$, the sum $\sum_{t} t\left(\omega_{n}\right)^{t\left(s-s^{\prime}\right)}$ 
may be formally identified as being proportional to a discrete derivative of the delta-function ${ }^{5}$ on $\mathbb{Z}_{q_{2 n}}$.

The relation (4.17) thereby identifies a finite shift operator $\Sigma$ acting on functions $f: \mathbb{Z}_{q_{2 n}} \rightarrow \mathbb{C}$. It may be regarded as the "infinitesimal" version of the shift operation in (4.19), according to the definition

$$
-\frac{2 \pi \mathrm{i}}{q_{2 n}} \sum_{s, t=1}^{q_{2 n}} t\left(\omega_{n}\right)^{t\left(s-s^{\prime}\right)} f(s):=\Sigma f\left(s^{\prime}\right) .
$$

In components this operator can be written as $\Sigma f\left(s^{\prime}\right)=\sum_{s} \Sigma_{s s^{\prime}} f(s)$ with

$$
\Sigma_{s s^{\prime}}=-\frac{2 \pi \mathrm{i}}{q_{2 n}} \sum_{t=1}^{q_{2 n}} t\left(\omega_{n}\right)^{t\left(s-s^{\prime}\right)} .
$$

For the action on matrices we define

$$
\left(\boldsymbol{\Sigma} \mathrm{a}_{n}\right)_{i j ; k}:=b_{i j ; k}^{(n)} .
$$

In components its action on matrix-valued functions on a circle is given by the expression $\boldsymbol{\Sigma} \mathrm{a}_{n}(\tau)_{i j}=\sum_{s, t} \boldsymbol{\Sigma}(\tau)_{i j, s t} \mathrm{a}_{n}(\tau)_{s t}$, with

$$
\boldsymbol{\Sigma}(\tau)_{i j, s t}=\Sigma_{i s} \times\left\{\begin{array}{ccc}
\delta_{t, s+j-i} & i<j & 1 \leq s \leq q_{2 n}+i-j \\
\delta_{t, s+j-i-q_{2 n}} \mathrm{e}^{2 \pi \mathrm{i} \tau} & i<j & q_{2 n}+i-j+1 \leq s \leq q_{2 n} \\
\delta_{t, s+i-j} & j \leq i & 1 \leq s \leq q_{2 n}+j-i \\
\delta_{t, s+i-j-q_{2 n}} \mathrm{e}^{-2 \pi \mathrm{i} \tau} & j<i & q_{2 n}+j-i+1 \leq s \leq q_{2 n}
\end{array}\right.
$$

The skew-adjoint shift operator $\boldsymbol{\Sigma}$ defines the finite analog of the derivative $\partial_{1}$ acting on the matrix part of the expansion (4.9).

Proceeding to the derivative $\nabla_{2}$ acting on the first tower in (4.8), by defining the "infinitesimal" clock operator

$$
\Xi_{i j}:=2 \pi \mathrm{i} j \delta_{i j}
$$

we may write the canonical matrix expansion coefficients $c_{i j ; k}^{(n)}$ of $j \alpha_{i+\left[\frac{q_{2 n}}{2}\right]_{0}, j ; k}^{(n)}$ using (4.14) and (4.16) as

$$
c_{i j ; k}^{(n)}=(j-i) a_{i j ; k}^{(n)}=\frac{1}{2 \pi \mathrm{i}}\left[\Xi, \mathrm{a}_{n}\right]_{i j ; k} .
$$

\footnotetext{
${ }^{5}$ To understand this identification better, it is instructive to recall the Fourier integral representation of the Dirac delta-function $\delta(x)=\int_{\mathbb{R}} \mathrm{d} k \mathrm{e}^{\mathrm{i} k x}$ on the real line $\mathbb{R}$. From this formula it follows that $\delta^{\prime}(x)=\mathrm{i} \int_{\mathbb{R}} \mathrm{d} k k \mathrm{e}^{\mathrm{i} k x}$ is the Fourier expansion of the derivative of the delta-function.
} 
The operator $\Xi$ defines the "infinitesimal" version of the clock operation in (4.19) and yields the finite analog of the derivative $\partial_{2}$ acting on the matrix part of the expansion (4.9). In this sense, the derivative terms in this matrix model are more akin to the derivatives obtained by expanding functions on the noncommutative plane in a soliton basis [54, 48, 11, 52, 33, 49].

Finally, the components of the derivations in (4.8) which are proportional to the circular Fourier integers $k$ are evidently proportional to the derivative operators $z \mathrm{~d} / \mathrm{d} z$ of $\mathbb{S}^{1}$ acting on $\mathrm{a}_{n}(z)$. Completely analogous formulæ hold also for the second tower in (4.8). In this way we may represent the derivatives (4.8) acting on matrix-valued functions (4.9) on $\mathbb{S}^{1}$ as

$$
\begin{aligned}
& \nabla_{1} \mathrm{a}_{n}\left(\tau, \tau^{\prime}\right)=\boldsymbol{\Sigma} \mathrm{a}_{n}(\tau) \oplus\left(q_{2 n-1} \dot{\mathrm{a}}_{n}^{\prime}\left(\tau^{\prime}\right)+\left[\Xi^{\prime}, \mathrm{a}_{n}^{\prime}\left(\tau^{\prime}\right)\right]\right), \\
& \nabla_{2} \mathrm{a}_{n}\left(\tau, \tau^{\prime}\right)=\left(q_{2 n} \dot{\mathrm{a}}_{n}(\tau)+\left[\Xi, \mathrm{a}_{n}(\tau)\right]\right) \oplus \boldsymbol{\Sigma}^{\prime} \mathrm{a}_{n}^{\prime}\left(\tau^{\prime}\right),
\end{aligned}
$$

where $\dot{\mathrm{a}}_{n}(\tau):=\mathrm{da} \mathrm{a}_{n}(\tau) / \mathrm{d} \tau$ and $\dot{\mathrm{a}}_{n}^{\prime}\left(\tau^{\prime}\right):=\mathrm{da}_{n}^{\prime}\left(\tau^{\prime}\right) / \mathrm{d} \tau^{\prime}$.

\subsection{Approximate Actions}

We can now write down an action defined on elements of $\mathcal{A}_{n}$ which approximates well the action functional (4.1) as

$$
\begin{aligned}
S_{n}= & \frac{g_{s} \mu_{9}}{G_{s}} \int_{\mathcal{M}} \sqrt{\operatorname{det} G}\left\{\beta_{2 n} \int_{0}^{1} \mathrm{~d} \tau \operatorname{Tr} \mathcal{L}\left[\boldsymbol{\Phi}_{n}(\tau), \nabla_{\mu} \boldsymbol{\Phi}_{n}(\tau)\right]\right. \\
& \left.+\beta_{2 n-1} \int_{0}^{1} \mathrm{~d} \tau^{\prime} \operatorname{Tr}^{\prime} \mathcal{L}\left[\boldsymbol{\Phi}_{n}^{\prime}\left(\tau^{\prime}\right), \nabla_{\mu} \boldsymbol{\Phi}_{n}^{\prime}\left(\tau^{\prime}\right)\right]\right\},
\end{aligned}
$$

with $\nabla_{\mu}, \mu=1,2$ given by (4.26). The noncommutativity of the torus has now been transformed into matrix noncommutativity. Note, however, that this is not the Morita equivalence of noncommutative field theories, which would connect a field theory on the noncommutative torus to a matrix theory on the regular torus $\mathbb{T}^{2}$. Here the matrix model is defined on a sum of two circles, and the procedure is exact in the limit, in the sense that the algebras $\mathcal{A}_{n}$ converge to $\mathcal{A}_{\theta}$ in the manner explained in the previous section. The fact that (4.27) already involves continuum fields is also the reason that the derivations (4.26) are infinitesimal versions of the usual lattice ones (4.19), and in the present case the removal of the matrix regularization does not require a complicated double scaling limit involving a small lattice spacing parameter. In the next section we shall study some explicit examples of this approximation to field theories on the noncommutative torus and, in particular, describe some aspects of their quantization in the matrix representation. 


\section{Applications}

In this section we will briefly describe three simple applications of the matrix quantum mechanics formalism. First, we shall analyse how the perturbative expansion of noncommutative field theories is described by the matrix model. We show that at any finite level $n$, there is no UV/IR mixing present in quantum amplitudes, but that the standard divergences are recovered in the limit $n \rightarrow \infty$. This suggests that the present matrix formalism could be a good arena to explore the renormalization of noncommutative field theories. Second, we examine a simple model for the energy density in string field theory. We show that the correct tension of a D-brane in processes involving tachyon condensation is already reproduced at a finite level in the matrix model. This feature fits well with the recent proposals on the description of tachyon dynamics in open string field theory, using one-dimensional matrix models for strings in two-dimensional target spaces [57, 55, 41, 58, 2]. Finally, we briefly initiate a nonperturbative analysis of gauge dynamics on the noncommutative torus by exploiting a Hamiltonian formulation of the matrix quantum mechanics, and indicate how the results compare with the known exact solution of noncommutative Yang-Mills theory in two dimensions [62]. More complicated exactly solvable models are also readily analysed in principle, in particular by exploiting the fact that the "time" direction of the matrix quantum mechanics is compactified on a circle so that the regulated theory is really a finite temperature field theory. For example, if one considers a $2+1$ dimensional field theory with space taken to be the noncommutative torus, then our regularization technique provides a dimensional reduction of the model to a $1+1$ dimensional matrix field theory with spacetime a cylinder $\mathbb{R} \times \mathbb{S}^{1}$.

\subsection{Perturbative Dynamics}

In this subsection we will demonstrate that perturbation theory within the framework of the matrix quantum mechanics is easily tractable, in contrast to some other matrix regularizations of noncommutative field theory, and show how various novel perturbative aspects arise within the matrix approximation scheme. For definiteness, we will concentrate on the real scalar $\phi^{4}$-theory which on the noncommutative torus is defined by the action

$$
S[\phi]=f\left[\frac{1}{2} \phi\left(\square+\mu^{2}\right) \phi+\frac{g}{4 !} \phi^{4}\right],
$$

where $\phi$ is a Hermitian element of the algebra $\mathcal{A}_{\theta}$ and $\square=\left(\partial_{1}\right)^{2}+\left(\partial_{2}\right)^{2}$ is the Laplacian, while $\mu$ and $g$ are respectively dimensionless mass and coupling 
parameters. Following the general prescription of the previous section, we approximate this field theory by the Hermitian matrix quantum mechanics with Euclidean action

$$
\begin{aligned}
& S_{n}\left[\boldsymbol{\phi}_{n}, \boldsymbol{\phi}_{n}^{\prime}\right]=\beta_{2 n} \int_{0}^{1} \mathrm{~d} \tau \operatorname{Tr}\left[\frac{1}{2} \boldsymbol{\phi}_{n}(\tau)\left(\left(\boldsymbol{\nabla}_{1}\right)^{2}+\left(\boldsymbol{\nabla}_{2}\right)^{2}+\mu^{2}\right) \boldsymbol{\phi}_{n}(\tau)\right. \\
& \left.\quad+\frac{g}{4 !} \boldsymbol{\phi}_{n}(\tau)^{4}\right] \\
& \quad+\beta_{2 n-1} \int_{0}^{1} \mathrm{~d} \tau^{\prime} \operatorname{Tr}^{\prime}\left[\frac{1}{2} \boldsymbol{\phi}_{n}^{\prime}\left(\tau^{\prime}\right)\left(\left(\nabla_{1}\right)^{2}+\left(\nabla_{2}\right)^{2}+\mu^{2}\right) \boldsymbol{\phi}_{n}^{\prime}\left(\tau^{\prime}\right)\right. \\
& \left.\quad+\frac{g}{4 !} \boldsymbol{\phi}_{n}^{\prime}\left(\tau^{\prime}\right)^{4}\right] .
\end{aligned}
$$

Everything we say in this subsection will hold independently and symmetrically in both towers of the finite level algebra $\mathcal{A}_{n}$, and so for brevity we will only analyse the first tower explicitly.

To deal with this quantum mechanics in perturbation theory, it is most convenient to use a power series expansion of the form (4.5) and expand the Hermitian scalar fields $\phi_{n} \in u\left(q_{2 n}\right) \otimes C^{\infty}\left(\mathbb{S}^{1}\right)$ in the first tower as

$$
\phi_{n}(z)=\sum_{i, j=0}^{q_{2 n}-1} \sum_{k \in \mathbb{Z}} \varphi_{i j ; k}^{(n)} z^{k}\left(\mathcal{C}_{q_{2 n}}\right)^{i}\left(\mathcal{S}_{q_{2 n}}(z)\right)^{j} .
$$

For the quantum theory, we will use path integral quantization, defined by treating the complex expansion coefficients $\varphi_{i j ; k}^{(n)}$ as the dynamical variables and integrating over the corresponding configuration space $\mathcal{C}_{n}:=u\left(q_{2 n}\right) \otimes$ $S(\mathbb{Z})$. Quantum correlation functions are then defined as

$$
\left\langle\varphi_{i_{1} j_{1} ; k_{1}}^{(n)} \cdots \varphi_{i_{L} j_{L} ; k_{L}}^{(n)}\right\rangle:=\frac{\int_{\mathcal{C}_{n}} \mathrm{D} \varphi^{(n)} \mathrm{e}^{-S_{n}\left[\varphi^{(n)}\right]} \varphi_{i_{1} j_{1} ; k_{1}}^{(n)} \cdots \varphi_{i_{L} j_{L} ; k_{L}}^{(n)}}{\int_{\mathcal{C}_{n}} \mathrm{D} \varphi^{(n)} \mathrm{e}^{-S_{n}\left[\varphi^{(n)}\right]}}
$$

where the integration measure is given by

$$
\mathrm{D} \varphi^{(n)}:=\prod_{i, j=0}^{q_{2 n}-1} \prod_{k \in \mathbb{Z}} \mathrm{d} \varphi_{i j ; k}^{(n)}
$$

with $\mathrm{d} \varphi_{i j ; k}^{(n)}$ ordinary Lebesgue measure on $\mathbb{C}$. Note that the expression (5.5) is still formal because of the infinitely many Fourier modes on $\mathbb{S}^{1}$. Nevertheless, as we show in the following, the finiteness of the range of the 
matrix indices in $\mathbb{Z}_{q_{2 n}}$ is sufficient to regularize the original noncommutative field theory.

We now substitute the expansion (5.3) into the action (5.2), compute derivatives using the definitions (4.8) and the $\left(\phi_{n}\right)^{4}$ interaction term using the commutation relations (3.24), and then apply the orthogonality relations (4.12). The quadratic form in the free part of the action (5.2) is then diagonal, and from its inverse we arrive at the free propagator

$$
\begin{aligned}
\triangle_{i j ; s t}^{(n) k l} & :=\left\langle\varphi_{i j ; k}^{(n) \dagger} \varphi_{s t ; l}^{(n)}\right\rangle_{g=0} \\
& =\frac{1}{(2 \pi)^{2} q_{2 n}\left(\beta_{2 n}\right)^{2}} \frac{1}{i^{2}+\left(j+q_{2 n} k\right)^{2}+\mu^{2}} \delta_{i s} \delta_{j t} \delta_{k l} .
\end{aligned}
$$

Furthermore, the vertices for the $\phi^{4}$ field theory in the matrix representation are given by

$$
\begin{aligned}
S_{n}\left[\varphi^{(n)}\right]_{\text {int }}= & \prod_{a=1}^{4} \sum_{\substack{i_{a}, j_{a}=0 \\
q_{2 n}-1}}^{q_{k_{a} \in \mathbb{Z}}} \varphi_{i_{1} j_{1} ; k_{1}}^{(n) \dagger} \varphi_{i_{2} j_{2} ; k_{2}}^{(n) \dagger} \varphi_{i_{3} j_{3} ; k_{3}}^{(n)} \varphi_{i_{4} j_{4} ; k_{4}}^{(n)} \\
& \times V_{i_{1} j_{1} ; i_{2} j_{2} ; i_{3} j_{3} ; i_{4} j_{4}}^{(n) k_{1}, k_{2}, k_{3}, k_{4}},
\end{aligned}
$$

where

$$
\begin{aligned}
V_{i_{1} j_{1} ; i_{2} j_{2} ; i_{3} j_{3} ; i_{4} j_{4}}^{(n) k_{1}, k_{2}, k_{3}, k_{4}}= & \frac{g}{4 !} q_{2 n}\left(\beta_{2 n}\right)^{2}\left(\omega_{n}\right)^{i_{3} j_{2}-i_{1} j_{4}} \\
& \times \delta_{i_{1}+i_{2}, i_{3}+i_{4}} \delta_{j_{1}+j_{2}, j_{3}+j_{4}} \delta_{k_{1}+k_{2}, k_{3}+k_{4}}
\end{aligned}
$$

The vertex function (5.8) is invariant under cyclic permutations of its arguments $\left(i_{a}, j_{a}, k_{a}\right), a=1, \ldots, 4$.

The propagator (5.6) in this representation is rather simple in form, in contrast to the usual matrix regularizations of noncommutative field theory wherein the kinetic terms of the action generically have a very complicated form $[5,54,49]$. This is what makes the matrix quantum mechanics approach much more fruitful. The matrix quantum mechanics provides an exact, finite regulated theory which precisely mimicks the properties of the original continuum model. This is a physical manifestation of the fact that the finite level algebras $\mathcal{A}_{n}$ converge to $\mathcal{A}_{\theta}$. In particular, from (5.6) and (5.8) we see that the Feynman graphs have a natural ribbon structure with an additional label by the circular momentum modes of the fields, and the notion of planarity in the matrix model is the same as that in the noncommutative field theory. Again, all of these features are in contradistinction to the usual matrix model formulations.

As an explicit example of how perturbation theory works within this setting, let us compute the quadratic part of the effective quantum action in 
the matrix representation. The one-loop dynamics is obtained by contracting two legs in (5.8) using the propagator (5.6). All eight possible contractions of two neighbouring legs are identical and sum up to give the total planar contribution

$$
8 \sum_{i^{\prime}, j^{\prime}=0}^{q_{2 n}-1} \sum_{s^{\prime}, t^{\prime}=0}^{q_{2 n}-1} \sum_{k^{\prime}, l^{\prime} \in \mathbb{Z}} \triangle_{i^{\prime} j^{\prime} ; s^{\prime} t^{\prime}}^{(n) k^{\prime} l^{\prime}} V_{i j ; i^{\prime} j^{\prime} ; s^{\prime} t^{\prime} ; s t}^{(n) k, k^{\prime}, l^{\prime}, l}=\frac{g}{3} \delta_{i s} \delta_{j t} \delta_{k l} I_{\mathrm{p}}^{(n)}\left(\mu^{2}\right)
$$

where

$$
I_{\mathrm{p}}^{(n)}\left(\mu^{2}\right)=\frac{1}{(2 \pi)^{2}} \sum_{i^{\prime}=0}^{q_{2 n}-1} \sum_{r \in \mathbb{Z}} \frac{1}{i^{2}+r^{2}+\mu^{2}} .
$$

In (5.10) we have transformed the sums over $j^{\prime} \in \mathbb{Z}_{q_{2 n}}$ and $k^{\prime} \in \mathbb{Z}$ into a single sum over $r:=j^{\prime}+q_{2 n} k^{\prime} \in \mathbb{Z}$. The infinite sum in (5.10) can be evaluated explicitly to give

$$
I_{\mathrm{p}}^{(n)}\left(\mu^{2}\right)=\frac{1}{4 \pi} \sum_{i^{\prime}=0}^{q_{2 n}-1} \frac{\operatorname{coth}\left(\pi \sqrt{i^{\prime 2}+\mu^{2}}\right)}{\sqrt{i^{\prime 2}+\mu^{2}}} .
$$

For any finite $q_{2 n}$ the function (5.11) is finite, for all $\mu^{2}$, and thus the matrix quantum mechanics naturally regulates the ultraviolet divergence of the one-loop scalar tadpole diagram. In the limit $n \rightarrow \infty$, whereby $q_{2 n} \rightarrow$ $\infty$, the sum (5.11) diverges, and the leading divergent behaviour can be straightforwardly worked out to be given by

$$
\lim _{n \rightarrow \infty} I_{\mathrm{p}}^{(n)}\left(\mu^{2}\right) \simeq \frac{1}{4 \pi} \ln \left(q_{2 n}\right)
$$

reproducing the standard logarithmic ultraviolet divergence of scalar field theory in two dimensions. In particular, we see that the matrix rank $q_{2 n}$ plays the role of an ultraviolet regulator in the matrix quantum mechanics. This is the characteristic feature of a fuzzy approximation to a field theory. In (5.11), the limit $q_{2 n} \rightarrow \infty$ requires an infinite mass renormalization $\mu=q_{2 n} \tilde{\mu}$, keeping $\tilde{\mu}$ fixed, in order to obtain a massive scalar field theory in the limit.

There are also four possible contractions of opposite legs in (5.8), which all agree and sum to give the total non-planar contribution

$$
4 \sum_{i^{\prime}, j^{\prime}=0}^{q_{2 n}-1} \sum_{s^{\prime}, t^{\prime}=0}^{q_{2 n}-1} \sum_{k^{\prime}, l^{\prime} \in \mathbb{Z}} \triangle_{i^{\prime} j^{\prime} ; s^{\prime} t^{\prime}}^{(n) k^{\prime} l^{\prime}} V_{i j ; i^{\prime} j^{\prime} ; s t ; s^{\prime} t^{\prime}}^{(n) k, k^{\prime}, l, l^{\prime}}=\frac{g}{6} \delta_{i s} \delta_{j t} \delta_{k l} I_{\mathrm{np}}^{(n)}\left(\mu^{2}\right)_{i j},
$$

where

$$
I_{\mathrm{np}}^{(n)}\left(\mu^{2}\right)_{i j}=\frac{1}{(2 \pi)^{2}} \sum_{i^{\prime}=0}^{q_{2 n}-1} \sum_{r \in \mathbb{Z}} \frac{\left(\omega_{n}\right)^{i^{\prime} j-i r}}{i^{\prime 2}+r^{2}+\mu^{2}}
$$


and we have used the fact that $\left(\omega_{n}\right)^{q_{2 n}}=1$. The infinite sum in (5.14) can be evaluated explicitly in terms of the generalized hypergeometric function

$$
{ }_{3} F_{2}\left\{\begin{array}{ccc}
\lambda_{1} & \lambda_{2} & \lambda_{3} \\
\eta_{1} & \eta_{2}
\end{array} \mid w\right\}:=\sum_{p \in \mathbb{N}_{0}} \frac{\left(\lambda_{1}\right)_{p}\left(\lambda_{2}\right)_{p}\left(\lambda_{3}\right)_{p}}{\left(\eta_{1}\right)_{p}\left(\eta_{2}\right)_{p}} \frac{w^{p}}{p !}
$$

with $w, \lambda_{a}, \eta_{b} \in \mathbb{C}, a=1,2,3, b=1,2$, and $(\lambda)_{p}:=\lambda(\lambda+1) \cdots(\lambda+p)$. One finds

$$
\begin{aligned}
& I_{\mathrm{np}}^{(n)}\left(\mu^{2}\right)_{i j}=\frac{1}{(2 \pi)^{2}} \frac{1}{\mu^{2}}{ }_{3} F_{2}\left\{\begin{array}{ccc}
1 & \mathrm{i} \mu & -\mathrm{i} \mu \\
1+\mathrm{i} \mu & 1-\mathrm{i} \mu
\end{array} \mid\left(\omega_{n}\right)^{j}\right\}
\end{aligned}
$$

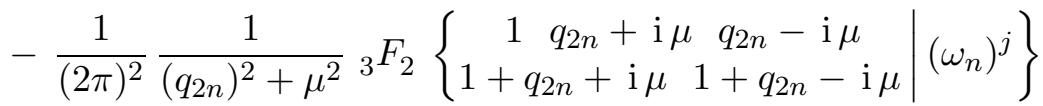

$$
\begin{aligned}
& +\frac{1}{2 \pi^{2}} \sum_{i^{\prime}=0}^{q_{2 n}-1} \frac{\left(\omega_{n}\right)^{2 j-i}}{1+i^{\prime 2}+\mu^{2}} \\
& \times{ }_{3} F_{2}\left\{\begin{array}{c}
11+\mathrm{i} \sqrt{i^{\prime 2}+\mu^{2}} 1-\mathrm{i} \sqrt{i^{\prime 2}+\mu^{2}} \\
2+\mathrm{i} \sqrt{i^{\prime 2}+\mu^{2}} 2-\mathrm{i} \sqrt{i^{\prime 2}+\mu^{2}}
\end{array} \mid\left(\omega_{n}\right)^{-i}\right\} .
\end{aligned}
$$

One can show from (5.15) that the leading large $n$ behaviour of (5.16) is given by

$$
\lim _{n \rightarrow \infty} I_{\mathrm{np}}^{(n)}\left(\mu^{2}\right)_{i j} \simeq \frac{1}{(2 \pi)^{2}\left(q_{2 n}\right)^{2}}\left(\frac{2\left(\omega_{n}\right)^{2 j}}{1-\left(\omega_{n}\right)^{i}}-\frac{1}{1-\left(\omega_{n}\right)^{j}}\right)
$$

with $\omega_{n} \rightarrow \mathrm{e}^{2 \pi \mathrm{i} \theta}$ in the limit. This quantity thereby vanishes, except when either $i=0, j=0$ or $\theta=0$ in which case it diverges. This is simply the UV/IR mixing property of the noncommutative field theory [60]. Integrating out infinitely many degrees of freedom in the non-planar loop diagram generates an infrared singularity, making the amplitude singular at vanishing external momentum and giving it a pole in the noncommutativity parameter at $\theta=0$.

On the other hand, at any finite level $n<\infty$, the non-planar matrix contribution is always finite. Generically, the generalized hypergeometric function (5.15) has a branch point at $w=1$, and for $\operatorname{Re}\left(\lambda_{1}+\lambda_{2}+\lambda_{3}-\right.$ $\left.\eta_{1}-\eta_{2}\right)<0$ the series is absolutely convergent everywhere on the unit disc $|w| \leq 1$. Thus for finite matrix rank $q_{2 n}$, the function (5.16) is an analytic function of the noncommutativity parameter, even for vanishing external momenta $i$ or $j$, i.e. there is no $\mathrm{UV} / \mathrm{IR}$ mixing in the matrix regulated theory, at least at one-loop order. Of course, there must be a transition regime in $q_{2 n}$ wherein a non-analyticity develops, as it appears in the limit 
(5.17). But integrating out all degrees of freedom in the loop does not generate an infrared singularity in the regulated model.

The absence of UV/IR mixing at finite level $n$, along with the simplicity of the propagator (5.6), implies that the matrix quantum mechanics is a good arena to explore the renormalizability of noncommutative field theories, as the usual mixing of high and low momentum scales would typically appear to make standard Wilsonian renormalization to all orders of perturbation theory hopeless. In particular, it confirms the expectations that an appropriate non-perturbative regularization could wash away these effects. On the other hand, it also seems to suggest that exotic non-perturbative phenomena, such as the existence of vacuum phases with broken translational symmetry [34], are unobservable at finite level. Indeed, for $n<\infty$ and generic $\mu^{2}<0$, there does not appear to be any qualitative difference in the infrared behaviour of the noncommutative propagator from the case $\mu^{2}>0$. It is most likely that there is again some transition regime for $n \gg 1$ wherein the exotic broken symmetry phases dominate the vacuum structure of the theory, and it would be interesting to find an analytic approach to detect these phases in the matrix quantum mechanics. Heuristically, their existence can be deduced by looking at the soliton expansions (4.9) of $\phi_{n}(z)$ in the finite level algebra $\mathcal{A}_{n}$ directly in terms of the projections and partial isometries of the noncommutative torus. Recall that these solitons displayed themselves momentum non-conserving stripe patterns (see figs. 2 and 3). A striped phase in the scalar field theory would then occur when, for $n$ sufficiently large, the mode numbers of the vacuum expectation value $\left\langle\varphi_{i j ; k}^{(n)}\right\rangle$ freeze about a particular value corresponding to a single projection or partial isometry $\mathrm{P}_{n}^{i j}$, and thereby yielding the characteristic stripe patterns. From this argument it is tempting to speculate that they may be due to a Kosterlitz-Thouless type phase transition in the matrix quantum mechanics which occurs in the large $n$ limit, whereby a condensation of vortices in the vacuum is responsible for the breaking of the translational symmetry.

Let us further remark that UV/IR mixing is also absent in the matrix model regularizations of noncommutative field theory that are derived by soliton expansion on the noncommutative plane [48, 49] (to be discussed in section 6.3), but not in those which are derived through lattice regularization. In these latter cases, UV/IR mixing is already present non-perturbatively as a generic kinematical property of the lattice regularization of noncommutative field theory [5]. Indeed, in the reduced models, striped phases of the theory are observable for relatively small values of the matrix dimension $[3,13]$. The relation between rational noncommutative theories and matrix-valued commutative theories on the torus is applied to UV/IR mix- 
ing in [35].

\subsection{Tachyon Dynamics}

We will now examine how the matrix quantum mechanics can be used to describe D-branes as the decay products in tachyon condensation on unstable D-branes in string field theory. We begin with the Type IIA case (equivalently bosonic strings) described in section 2.2. We are interested in the noncommutative field theory of the open string tachyon and gauge field on a system of unstable D9-branes. This depends on the specification of a projective module over $\mathcal{A}_{\theta}$ (see section 6.1) in order to define the anti-Hermitian connection gauge field $A_{\mu}$, but for simplicity we consider here only the free module $\left(\mathcal{A}_{\theta}\right)^{\oplus N}$ provided by $N$ copies of the noncommutative torus algebra itself, which corresponds to a topologically trivial connection on the worldvolume field theory of $N$ noncommutative D9-branes. The components of the curvature of the gauge connection are denoted $F_{\mu \nu}$. The tachyon field is Hermitian and lives in the adjoint representation of the gauge group, and its covariant derivatives are denoted $D_{\mu} T$.

The action is given explicitly by $[38,37]$

$$
\begin{aligned}
& S^{\mathrm{IIA}}=\frac{g_{s} \mu_{9}}{G_{s}} \int_{\mathcal{M}} \sqrt{\operatorname{det} G} f\left[\frac{1}{2} f\left(T^{2}-\mathbb{1}\right) G^{\mu \nu} D_{\mu} T D_{\nu} T-V\left(T^{2}-\mathbb{1}\right)\right. \\
& \left.-\frac{1}{4} h\left(T^{2}-\mathbb{1}\right) F_{\mu \nu} F^{\mu \nu}+\ldots\right],
\end{aligned}
$$

where here and in the following repeated indices are always understood to be summed over, and indices are raised by the inverse open string metric $G^{\mu \nu}$. The dots in (5.18) denote possible higher-derivative contributions to the effective action, but will not be required in the ensuing low-energy analysis. The functions $f$ and $h$, and the tachyon potential $V$, are not known explicitly, but they are constrained to satisfy certain conditions in accord with the conjectures surrounding open string tachyon condensation [73]. In particular, the tachyon potential has a local maximum at $T=0$ representing the unstable D9-branes, with $\mu_{9}=V(-1)$ giving their tension. It also has local minima $V(0)=0$ at $T= \pm \mathbb{1}$ corresponding to the closed string vacuum. The functions $f$ and $h$ vanish in the closed string vacuum, $f(0)=h(0)=0$, while $f(-1)=h(-1)=1$.

We will consider a simplified version of this model to make the results as transparent as possible. We study a $2+1$-dimensional noncommutative field theory, i.e. take $\mathcal{M}=\mathbb{R}^{1}$, and consider the action near $T=0$. The 
corresponding energy functional

$$
E^{\mathrm{IIA}}[A, T]=f\left[\frac{1}{2} D_{\mu} T D_{\mu} T+V\left(T^{2}-\mathbb{1}\right)+\frac{1}{2} F^{2}\right]
$$

is then that of a D2-brane wrapped around $\mathbb{T}^{2}$ in the presence of a constant $B$-field, with $D_{\mu} T=\partial_{\mu} T-\left[A_{\mu}, T\right], \mu=1,2$ and

$$
F=\partial_{1} A_{2}-\partial_{2} A_{1}+\left[A_{1}, A_{2}\right]
$$

The simplest classical extrema of (5.19) are given by spatially uniform tachyon fields $\Omega^{-1}(T)$ on $\mathbb{T}^{2}\left(\Omega^{-1}\right.$ being the Wigner map (2.6)) which are critical points of the potential $V\left(T^{2}-1\right)$, and vanishing gauge fields $F=A_{\mu}=0$, $\mu=1,2$. We will now proceed to analyse these vacua within the matrix approximation. In the Hermitian matrix quantum mechanics, we replace the energy functional (5.19) by the Euclidean action

$$
\begin{aligned}
E_{n}^{\mathrm{IIA}}\left[\mathrm{T}_{n}, \mathrm{~T}_{n}^{\prime}\right]= & \beta_{2 n} \int_{0}^{1} \mathrm{~d} \tau \operatorname{Tr}\left[\frac{1}{2} \nabla_{\mu} \mathrm{T}_{n}(\tau) \nabla_{\mu} \mathrm{T}_{n}(\tau)+V\left(\mathrm{~T}_{n}(\tau)^{2}-\mathbb{1}_{q_{2 n}}\right)\right] \\
& +\beta_{2 n-1} \int_{0}^{1} \mathrm{~d} \tau^{\prime} \operatorname{Tr}^{\prime}\left[\frac{1}{2} \nabla_{\mu} \mathrm{T}_{n}^{\prime}\left(\tau^{\prime}\right) \nabla_{\mu} \mathrm{T}_{n}^{\prime}\left(\tau^{\prime}\right)\right. \\
& \left.+V\left(\mathrm{~T}_{n}^{\prime}\left(\tau^{\prime}\right)^{2}-\mathbb{1}_{q_{2 n-1}}\right)\right] .
\end{aligned}
$$

Focusing for the time being on the first tower, we shall seek time independent extrema of the energy functional (5.21), $\dot{\mathrm{T}}_{n}=0$. From (4.24) and (4.26) it then follows that the $q_{2 n}\left(q_{2 n}+1\right) / 2$ equations for the critical points are given by

$$
-(2 \pi)^{2}(i-j)^{2}\left(\mathbf{T}_{n}\right)_{i j}-\left(\boldsymbol{\Sigma} \mathbf{T}_{n} \boldsymbol{\Sigma}\right)_{i j}=\left(\mathbf{T}_{n} V^{\prime}\left(\left(\mathbf{T}_{n}\right)^{2}-\mathbb{1}_{q_{2 n}}\right)\right)_{i j}
$$

for $1 \leq i \leq j \leq q_{2 n}$. We have used the fact that both $\nabla_{1}$ and $\nabla_{2}$ satisfy an "integration by parts" rule

$$
\operatorname{Tr}\left(\mathrm{a}_{n}^{\dagger}\left(\nabla_{\mu} \mathrm{b}_{n}\right)\right)=-\operatorname{Tr}\left(\left(\nabla_{\mu} \mathrm{a}_{n}\right)^{\dagger} \mathrm{b}_{n}\right), \quad \mu=1,2 .
$$

It is straightforward to see from these equations that the off-diagonal elements of the matrix $\mathrm{T}_{n}$ vanish. This follows from the explicit form (4.23) of the shift operator $\boldsymbol{\Sigma}$, which for $i<j$ would produce a $\tau$-dependence in the second term of (5.22), while the other two terms are time-independent. 
Thus $\mathrm{T}_{n}$ must be a diagonal matrix, which we write explicitly in terms of projections on $\mathcal{A}_{\theta}$ as

$$
\mathrm{T}_{n}=\sum_{i=1}^{q_{2 n}} \eta_{i} \mathrm{P}_{n}^{i i}
$$

The moduli $\eta_{i} \in \mathbb{R}$ of this solution have constraints which may be found by substituting (5.24) into (5.22), using (4.23) along with the fact that $\boldsymbol{\Sigma}_{i j, s s} \neq 0$ only if $i=j$, and by using $\operatorname{Tr} \boldsymbol{\Sigma}=\pi \mathrm{i}\left(q_{2 n}+1\right)$. We can thus write the equation for the $\eta_{i}$ 's as

$$
\eta_{i} V^{\prime}\left(\left(\eta_{i}\right)^{2}-1\right)=\pi^{2}\left(q_{2 n}+1\right)^{2} \sum_{j=1}^{q_{2 n}} \eta_{j}
$$

which must be satisfied for each $i=1, \ldots, q_{2 n}$.

This result is fairly generic. It states that time-independent field configurations on the noncommutative torus correspond to diagonal matrices in the matrix quantum mechanics. In particular, all classical ground states commute with each other. This is reminescent of what happens in the BFSS matrix model of M-theory [8], whereby the vacuum corresponds to static, commuting spacetime matrix coordinates for D0-branes. Moreover, this solution shows that the time-independent configurations of the matrix quantum mechanics are naturally projection-type solitons on the noncommutative torus. We will see in the next section how projections on $\mathcal{A}_{\theta}$ also arise by a somewhat different dynamical mechanism.

A class of solutions to the equations (5.25) can be constructed by demanding that $\mathrm{T}_{n}$ be a critical point of the tachyon potential $V\left(T^{2}-\mathbb{1}\right)$, i.e. $\mathrm{T}_{n} V^{\prime}\left(\left(\mathrm{T}_{n}\right)^{2}-\mathbb{1}_{q_{2 n}}\right)=0$. For this, we assume that $n$ is sufficiently large, that the matrix dimension $q_{2 n}$ is even, and that $V(\lambda)$ is a polynomial potential. Let $\left\{\lambda_{I}\right\}_{I \geq 1}$ be a set of distinct, real critical points of $V(\lambda)$ which are each bounded from below as $\lambda_{I} \geq-1$. We then arrange the collection of real numbers $\left\{\eta_{j}\right\}_{j=1}^{q_{2 n}}$ pairwise according to the rule

$$
\eta_{I}=\sqrt{1+\lambda_{I}}, \quad \eta_{q_{2 n}-I+1}=-\sqrt{1+\lambda_{I}}, \quad I=1,2, \ldots
$$

with the remaining $\eta_{i}$ 's set equal to \pm 1 in pairs. The solution (5.24) then obeys

$$
\left(\mathrm{T}_{n}\right)^{2}-\mathbb{1}_{q_{2 n}}=\sum_{I \geq 1} \lambda_{I}\left(\mathrm{P}_{n}^{I I}+\mathrm{P}_{n}^{q_{2 n}-I+1, q_{2 n}-I+1}\right)
$$

and thereby satisfies the required extremization condition. In this case, both sides of (5.25) vanish.

The energy of these classical solutions in the matrix quantum mechanics may now be found by substituting (5.27) into (5.21). An identical analysis 
proceeds in the second tower, producing a solution parametrized by another set $\left\{\lambda_{I^{\prime}}^{\prime}\right\}_{I^{\prime} \geq 1}$ of critical points. After recalling the definition of the sequence $\beta_{k}$ from appendix $\mathrm{A}$, one finds

$$
E_{n}^{\mathrm{IIA}}\left\{\lambda_{I}, \lambda_{I^{\prime}}^{\prime}\right\}=2\left(p_{2 n-1}-q_{2 n-1} \theta\right) \sum_{I} V\left(\lambda_{I}\right)-2\left(p_{2 n}-q_{2 n} \theta\right) \sum_{I^{\prime}} V\left(\lambda_{I^{\prime}}^{\prime}\right)
$$

The lightest excitation corresponds to the configuration whereby all $\lambda_{I}$ 's and $\lambda_{I^{\prime}}^{\prime}$ 's vanish except $\lambda_{1}=-1$. Then this formula gives the standard contribution to the mass-shell relation from the tension $\mu_{2}=V(-1)$ of the D2-branes, as can be found from the appropriate Born-Infeld action for the D-brane dynamics. What is remarkable about this term is that it has the correct $\theta$-dependence already at a finite level in the matrix model. Since the induced mass of a D0-brane bound to the D2-branes due to the background $B$-field is given by $\mu_{0}=\theta \mu_{2}$, the term $\left(p_{2 n-1}-q_{2 n-1} \theta\right) V(-1)$ arising in this way from (5.28) represents the energy of $p_{2 n-1}$ D2-branes carrying $-q_{2 n-1}$ units of D0-brane monopole charge. Thus at any finite level $n$, it gives the appropriate mass-shell relation on the noncommutative torus with energy bounded between 0 and $\mu_{2}$.

Let us now turn to the Type IIB case. Following the prescription of section 2.2, the appropriate version of the string field theory action (5.18) can be written down [37]. By using the same steps as above, the analog of the regulated energy functional (5.21) in the first tower reads

$$
\begin{aligned}
E_{n}^{\mathrm{IIB}}\left[\mathrm{T}_{n}\right]=\beta_{2 n} & \int_{0}^{1} \mathrm{~d} \tau \operatorname{Tr}\left[\left(\nabla_{\mu} \mathrm{T}_{n}(\tau)\right)^{\dagger}\left(\nabla_{\mu} \mathrm{T}_{n}(\tau)\right)\right. \\
& \left.+U\left(\mathrm{~T}_{n}(\tau)^{\dagger} \mathrm{T}_{n}(\tau)-\mathbb{1}_{r_{2 n}}\right)+U\left(\mathrm{~T}_{n}(\tau) \mathrm{T}_{n}(\tau)^{\dagger}-\mathbb{1}_{q_{2 n}}\right)\right]
\end{aligned}
$$

where now the regulated tachyon field $\mathrm{T}_{n}(\tau)$ is a $q_{2 n} \times r_{2 n}$ complex-valued matrix, with $\mathrm{T}_{n}(\tau)^{\dagger}$ its Hermitian conjugate. This functional describes an approximation to the noncommutative field theory of a D2 brane-antibrane system wrapping $\mathbb{T}^{2}$. We will take the arbitrary integers $r_{2 n} \leq q_{2 n}$ for definiteness, with $r_{2 n} \rightarrow \infty$ in the limit $n \rightarrow \infty$. Varying (5.29) on timeindependent configurations $\dot{\mathrm{T}}_{n}=0$ yields the critical point equations

$$
\begin{aligned}
& -(2 \pi)^{2}(i-j)^{2}\left(\mathrm{~T}_{n}\right)_{i j}+\left(\boldsymbol{\Sigma}^{2} \mathrm{~T}_{n}\right)_{i j} \\
& \quad=\left(\mathrm{T}_{n} U^{\prime}\left(\mathrm{T}_{n}^{\dagger} \mathrm{T}_{n}-\mathbb{1}_{r_{2 n}}\right)+U^{\prime}\left(\mathrm{T}_{n} \mathrm{~T}_{n}^{\dagger}-\mathbb{1}_{q_{2 n}}\right) \mathrm{T}_{n}\right)_{i j}
\end{aligned}
$$

with $1 \leq i \leq q_{2 n}$ and $1 \leq j \leq r_{2 n}$, plus the analogous equations for the conjugate matrix elements $\left(\mathrm{T}_{n}^{\dagger}\right)_{i j}$. 
As before, it is straightforward to show from (5.30) that all $i \neq j$ matrix elements of $\mathrm{T}_{n}$ vanish. We can thereby write down solutions as the $q_{2 n} \times r_{2 n}$ complex matrices

$$
\mathrm{T}_{n}=\left(\begin{array}{c}
\sum_{i=1}^{r_{2 n}} \sigma_{i} \mathrm{P}_{n}^{i i} \\
(0)_{\left(q_{2 n}-r_{2 n}\right) \times r_{2 n}}
\end{array}\right)
$$

of generic rank $r_{2 n}$ with

$$
\mathrm{T}_{n}^{\dagger} \mathrm{T}_{n}=\sum_{i=1}^{r_{2 n}}\left|\sigma_{i}\right|^{2} \mathrm{P}_{n}^{i i}, \quad \mathrm{~T}_{n} \mathrm{~T}_{n}^{\dagger}=\sum_{i=1}^{r_{2 n}}\left|\sigma_{i}\right|^{2} \mathrm{P}_{n}^{i i} \oplus(0)_{q_{2 n} \times q_{2 n}}
$$

where the moduli $\sigma_{i} \in \mathbb{C}$ satisfy an equation completely analogous to (5.25). Generically, these solutions are evidently determined by finite-dimensional partial isometries on $\mathbb{C}^{r_{2 n}} \rightarrow \mathbb{C}^{q_{2 n}}$. By taking $\left|\sigma_{i}\right|=\eta_{i}$, with $\eta_{i}$ as in (5.26), and substituting $(5.31,5.32)$ into $(5.29)$, we find that the energy of this solution is given by

$$
E_{n}^{\mathrm{IIB}}\left[\mathrm{T}_{n}\right]=\beta_{2 n}\left(4 \sum_{I} U\left(\lambda_{I}\right)+\left(q_{2 n}-r_{2 n}\right) U(-1)\right) .
$$

When $r_{2 n}=q_{2 n}$, the energy (5.33) is precisely twice that of the Type IIA case (5.28). By adjusting parameters as before, this is the energy appropriate to $q_{2 n-1}$ D0-branes and $q_{2 n-1}$ D0-antibranes inside the original D2- $\overline{\mathrm{D} 2}$ system. For $r_{2 n}<q_{2 n}$, the second term of (5.33) dominates in the limit $n \rightarrow \infty$. From appendix A, eqs. (A.5) and (A.8), we have $\beta_{2 n} \simeq 1 / q_{2 n-1}$ in the large $n$ limit, so that by taking $r_{2 n} \simeq q_{2 n}$ in this limit, we may adjust the second term so that it yields the appropriate continuum mass-shell relation for the D0 brane-antibrane system in the D2- $\overline{\mathrm{D} 2}$ system. Furthermore, from (5.32) it follows that, for generic moduli $\sigma_{i} \in \mathbb{C}$, the index of the regulated tachyon field configuration is given by

$$
\operatorname{index}\left(\mathrm{T}_{n}\right)=\operatorname{Tr}\left(\mathbb{1}_{r_{2 n}}-\mathrm{T}_{n}^{\dagger} \mathrm{T}_{n}\right)-\operatorname{Tr}\left(\mathbb{1}_{q_{2 n}}-\mathrm{T}_{n} \mathrm{~T}_{n}^{\dagger}\right)=r_{2 n}-q_{2 n},
$$

and thus it carries the correct monopole charge of $q_{2 n}$ D0-branes and $r_{2 n}$ D0-antibranes.

We conclude that the finite-level matrix quantum mechanics captures quantitative properties of D-brane projection solitons in open string field theory, through the standard mechanism of tachyon condensation on unstable D-branes. Heavier excitations correspond to more complicated configurations of D0-branes in the matrix model. It would be interesting to characterize also time-dependent solutions of the matrix quantum mechanics. In this context, the classical ground states may mimick those of the 
BMN matrix model for M-theory in a plane wave background [12], which admits a multitude of supersymmetric time-dependent classical configurations. A particularly interesting class of finite-dimensional $\frac{1}{2}$-BPS configurations describes rotating non-spherical giant gravitons with the noncommutative geometry of a fuzzy torus $[59,64]$. The solution depends on two moduli $\mu, \zeta \in \mathbb{R}$ and is given explicitly by

$$
\mathrm{Z}_{n}(\tau)=\mathrm{e}^{\mu \tau / 3}\left(\sum_{i=1}^{q_{2 n}-1} \alpha_{i} \mathrm{P}_{n}^{i, i+1}+\alpha_{q_{2 n}} \mathrm{P}_{n}^{q_{2 n}, 1}\right)
$$

where the parameters $\alpha_{i}(\mu, \zeta) \in \mathbb{C}$ are constrained by the pertinent BPS equations. Time-dependent solutions are thereby expected to dynamically generate off-diagonal elements of the soliton basis.

\subsection{Yang-Mills Matrix Quantum Mechanics}

Let $p, q>0$ be a pair of relatively prime integers, and $\mathcal{E}_{p, q}$ a Heisenberg module over a "dual" noncommutative torus $\mathcal{A}_{\alpha}$ to $\mathcal{A}_{\theta}{ }^{6}$. Choose a connection on $\mathcal{E}_{p, q}$ with corresponding anti-Hermitian gauge fields $A_{\mu} \in \mathcal{A}_{\theta}, \mu=1,2$ and curvature given by (5.20). Yang-Mills gauge theory on $\mathcal{A}_{\alpha}$ is then defined by the classical action

$$
S_{\mathrm{YM}}\left[A_{1}, A_{2}\right]=\frac{1}{2 g^{2}} f F^{2},
$$

with $g$ the dimensionless Yang-Mills coupling constant. The corresponding matrix quantum mechanics is the one-dimensional field theory of four antiHermitian matrix fields with action

$$
\begin{aligned}
S_{n}\left[\mathrm{X}_{n}, \mathrm{Y}_{n} ; \mathrm{X}_{n}^{\prime}, \mathrm{Y}_{n}^{\prime}\right]= & \frac{\beta_{2 n}}{2 g^{2}} \int_{0}^{1} \mathrm{~d} \tau \operatorname{Tr}\left(\nabla_{1} \mathrm{X}_{n}(\tau)-\nabla_{2} \mathrm{Y}_{n}(\tau)\right. \\
& \left.+\left[\mathrm{X}_{n}(\tau), \mathrm{Y}_{n}(\tau)\right]\right)^{2} \\
& +\frac{\beta_{2 n-1}}{2 g^{2}} \int_{0}^{1} \mathrm{~d} \tau^{\prime} \operatorname{Tr}^{\prime}\left(\nabla_{1} \mathrm{X}_{n}^{\prime}\left(\tau^{\prime}\right)-\nabla_{2} \mathrm{Y}_{n}^{\prime}\left(\tau^{\prime}\right)\right. \\
& \left.+\left[\mathrm{X}_{n}^{\prime}\left(\tau^{\prime}\right), \mathrm{Y}_{n}^{\prime}\left(\tau^{\prime}\right)\right]\right)^{2} .
\end{aligned}
$$

In this subsection we will exploit the fact that the time direction of the matrix quantum mechanics (5.37) is Euclidean and compactified on the

\footnotetext{
${ }^{6}$ These projective modules and dual algebras will be described explicitly in section 6.1 .
} 
unit circle $\mathbb{S}^{1}$. This implies that the corresponding path integrals compute quantum averages of the system in a thermal ensemble. The vacuum energy, for example, is given by the usual statistical mechanical partition function

$$
\mathcal{Z}_{n}=\operatorname{Tr}_{\mathcal{G}}\left(\mathrm{e}^{-\hat{H}_{n}}\right)=\sum_{\lambda_{n} \in \operatorname{spec}\left(\hat{H}_{n}\right)} \mathrm{e}^{-\lambda_{n}}
$$

where the trace is over the Hilbert space $\mathcal{G}$ of physical states of the matrix quantum mechanics and $\hat{H}_{n}$ is the quantum Hamiltonian operator, represented on $\mathcal{G}$, corresponding to the action (5.37). The partition function may thereby be readily obtained by computing the eigenvalues of $\hat{H}_{n}$ in canonical quantization of the model (5.37) on $\mathbb{R}$. Quantum gauge theory on the noncommutative torus is known to be an exactly solvable model which is given exactly by its semi-classical approximation [62]. In the following we will study the manner in which its matrix approximation captures this feature. While we will not completely solve the problem at the level of the matrix quantum mechanics, our analysis will illustrate in a straightforward manner what properties to seek in the search for exactly solvable noncommutative field theories. Analogous computations for the lattice regularizations of noncommutative gauge theory in two dimensions can be found in $[63,32]$.

As always, we focus on the first tower in (5.37), and use (4.26) to write the action explicitly as

$$
S_{n}\left[\mathrm{X}_{n}, \mathrm{Y}_{n}\right]=\frac{\beta_{2 n}}{2 g^{2}} \int_{0}^{1} \mathrm{~d} \tau \operatorname{Tr}\left(\boldsymbol{\Sigma} \mathbf{X}_{n}(\tau)-q_{2 n} \dot{\mathrm{Y}}_{n}(\tau)+\left[\mathrm{X}_{n}(\tau)-\Xi, \mathrm{Y}_{n}(\tau)\right]\right)^{2}
$$

The canonical momentum conjugate to $\mathrm{Y}_{n}$ is given by

$$
\left(\mathbf{\Pi}_{n}\right)_{i j}:=\frac{\delta S_{n}}{\delta\left(\dot{\mathrm{Y}}_{n}\right)_{i j}}=-\frac{q_{2 n} \beta_{2 n}}{g^{2}}\left(\boldsymbol{\Sigma} \mathbf{X}_{n}-q_{2 n} \dot{\mathrm{Y}}_{n}+\left[\mathrm{X}_{n}-\Xi, \mathbf{Y}_{n}\right]\right)_{j i}
$$

while the momentum of the $\mathrm{X}_{n}$ field vanishes since (5.39) involves no time derivatives of $X_{n}$. The matrix field $X_{n}$ is thus non-dynamical and serves simply as a Lagrange multiplier imposing the constraints $\frac{\delta S_{n}}{\delta\left(\mathrm{X}_{n}\right)_{i j}}=0$ for $1 \leq i, j \leq q_{2 n}$. By using (5.40) these constraint equations may be written in the simple matrix form

$$
\mathrm{G}_{n}:=\boldsymbol{\Sigma} \boldsymbol{\Pi}_{n}+\left[\mathrm{Y}_{n}, \boldsymbol{\Pi}_{n}\right]=0 .
$$

The Hamiltonian corresponding to the action (5.39) is given using (5.40) as

$$
H_{n}=\operatorname{Tr}\left(\frac{g^{2}}{2\left(q_{2 n}\right)^{2} \beta_{2 n}}\left(\mathbf{\Pi}_{n}\right)^{2}+\frac{1}{q_{2 n}}\left(\boldsymbol{\Sigma} \mathbf{X}_{n}+\left[\mathbf{X}_{n}-\Xi, \mathbf{Y}_{n}\right]\right) \mathbf{\Pi}_{n}\right)
$$


which after imposing the constraints (5.41) and using cyclicity of the trace can be written as

$$
H_{n}=\operatorname{Tr}\left(\frac{g^{2}}{2\left(q_{2 n}\right)^{2} \beta_{2 n}}\left(\boldsymbol{\Pi}_{n}\right)^{2}-\frac{1}{q_{2 n}}\left[\Xi, \mathbf{Y}_{n}\right] \mathbf{\Pi}_{n}\right) .
$$

Note that the constraints (5.41) are explicitly time-dependent, while the Hamiltonian (5.43) on the constraint surface is independent of $\tau$.

In canonical quantization, we promote the matrix fields $\mathbf{Y}_{n}, \boldsymbol{\Pi}_{n}$ to operators obeying the commutation relations

$$
\left[\left(\boldsymbol{\Pi}_{n}\right)_{i j},\left(\mathrm{Y}_{n}\right)_{k l}\right]=-\mathrm{i} \delta_{i k} \delta_{j l}
$$

We will represent (5.44) in the Schrödinger polarization wherein the physical states are the wavefunctions $\Psi\left(\mathrm{Y}_{n}\right) \in L^{2}\left(\mathrm{i} u\left(q_{2 n}\right)\right)=L^{2}\left(\mathbb{R}^{\left(q_{2 n}\right)^{2}}\right):=\mathcal{G}$ and the canonical momenta are represented as the derivative operators

$$
\left(\boldsymbol{\Pi}_{n}\right)_{i j}=-\mathrm{i} \frac{\partial}{\partial\left(\mathrm{Y}_{n}\right)_{i j}}
$$

acting on $\mathcal{G}$. By choosing a normal ordering prescription, the quantum Hamiltonian operator on $\mathcal{G}$ is then given from (5.43) as

$$
\hat{H}_{n}=\sum_{i, j=1}^{q_{2 n}}\left(-\frac{g^{2}}{2\left(q_{2 n}\right)^{2} \beta_{2 n}} \frac{\partial^{2}}{\partial\left(\mathrm{Y}_{n}\right)_{i j} \partial\left(\mathrm{Y}_{n}\right)_{j i}}+\frac{2 \pi}{q_{2 n}}(i-j)\left(\mathrm{Y}_{n}\right)_{i j} \frac{\partial}{\partial\left(\mathrm{Y}_{n}\right)_{j i}}\right)
$$

The constraints (5.41) should now be implemented on the Hilbert space $\mathcal{G}$, which truncates it to the subspace of physical wavefunctions $\Psi$ obeying

$$
\mathrm{G}_{n} \Psi=0 \text {. }
$$

From (5.45) and the explicit form (4.23) of the shift operator, we thereby arrive at the set of $\left(q_{2 n}\right)^{2}$ differential equations

$$
\begin{aligned}
& {\left[\sum_{s=1}^{q_{2 n}+i-j} \Sigma_{i s} \frac{\partial}{\partial\left(\mathrm{Y}_{n}\right)_{s, s+j-i}}+\mathrm{e}^{2 \pi \mathrm{i} \tau} \sum_{s=q_{2 n}+i-j+1}^{q_{2 n}} \sum_{i s} \frac{\partial}{\partial\left(\mathrm{Y}_{n}\right)_{s, s+j-i-q_{2 n}}}\right.} \\
& \left.\quad+\sum_{s=1}^{q_{2 n}}\left(\left(\mathrm{Y}_{n}\right)_{i s} \frac{\partial}{\partial\left(\mathrm{Y}_{n}\right)_{s j}}-\left(\mathrm{Y}_{n}\right)_{s j} \frac{\partial}{\partial\left(\mathrm{Y}_{n}\right)_{i s}}\right)\right] \Psi\left(\mathrm{Y}_{n}\right)=0
\end{aligned}
$$

for $i<j$, and

$$
\begin{aligned}
& {\left[\sum_{s=1}^{q_{2 n}+j-i} \Sigma_{i s} \frac{\partial}{\partial\left(\mathrm{Y}_{n}\right)_{s, s+i-j}}+\mathrm{e}^{-2 \pi \mathrm{i} \tau} \sum_{s=q_{2 n}+j-i+1}^{q_{2 n}} \Sigma_{i s} \frac{\partial}{\partial\left(\mathrm{Y}_{n}\right)_{s, s+i-j-q_{2 n}}}\right.} \\
& \left.\quad+\sum_{s=1}^{q_{2 n}}\left(\left(\mathrm{Y}_{n}\right)_{i s} \frac{\partial}{\partial\left(\mathrm{Y}_{n}\right)_{s j}}-\left(\mathrm{Y}_{n}\right)_{s j} \frac{\partial}{\partial\left(\mathrm{Y}_{n}\right)_{i s}}\right)\right] \Psi\left(\mathrm{Y}_{n}\right)=0
\end{aligned}
$$


for $i \geq j$. These constraints have to be imposed at all times $\tau$. With the understanding that the $i=j$ part is set trivially to 0 , the $\tau$-dependent parts of $(5.48,5.49)$ combine into the constraints

$$
\sum_{s=q_{2 n}-|i-j|+1}^{q_{2 n}} \Sigma_{i s} \frac{\partial \Psi\left(\mathrm{Y}_{n}\right)}{\partial\left(\mathrm{Y}_{n}\right)_{s, s+|i-j|-q_{2 n}}}=0
$$

which hold for all $i, j=1, \ldots, q_{2 n}$. One can show from (4.21) that the shift matrix $\left(\Sigma_{s s^{\prime}}\right)_{1 \leq s, s^{\prime} \leq q_{2 n}}$ is invertible. It follows then from (5.50) that the physical wavefunctions $\Psi\left(\mathrm{Y}_{n}\right)$ are independent of the off-diagonal elements $\left(\mathrm{Y}_{n}\right)_{i j}$ for $i<j$. By anti-Hermiticity, they are also independent of $\left(\mathrm{Y}_{n}\right)_{i j}=$ $-\overline{\left(\mathrm{Y}_{n}\right)_{j i}}$ for $i>j$. By setting $i=j$ in (5.49) the same argument shows that $\Psi\left(\mathrm{Y}_{n}\right)$ are independent of all diagonal matrix elements of $\mathrm{Y}_{n}$, and thus must vanish in $L^{2}\left(\mathrm{i} u\left(q_{2 n}\right)\right)$.

It follows that there are no physical propagating modes left in the quantum theory, and the quantum Hamiltonian (5.46) vanishes on the physical state space, $\hat{H}_{n}=0$. This feature is the earmark of a topological quantum field theory in which only global, topological degrees of freedom play a role. It is exactly what is anticipated in two-dimensional noncommutative Yang-Mills theory [62], whereby the gauge invariance of the theory under area-preserving diffeomorphisms of $\mathbb{T}^{2}$ kills all local degrees of freedom in the model. We may take the present analysis in the matrix model to be a direct proof of the topological nature of noncommutative gauge theory in two dimensions.

While this feature would appear to make the statistical sum (5.38) trivial, this is not the case, as there is a large moduli space of field configurations obeying the constraints (5.41). The continuum version of the quantum theory is given exactly by the semi-classical expansion, and we would expect the matrix regularization to capture this property in some way. For this, we write the thermal partition function (5.38) explicitly as the formal path integral

$$
\mathcal{Z}_{n}=\int_{\left(\mathcal{C}_{n}\right)^{2}} \mathrm{DX}_{n} \mathrm{DY}_{n} \mathrm{e}^{-S_{n}\left[\mathrm{X}_{n}, \mathrm{Y}_{n}\right]},
$$

where the configuration space is $\mathcal{C}_{n}:=\mathrm{i} u\left(q_{2 n}\right) \otimes C^{\infty}\left(\mathbb{S}^{1}\right)$ and the integration measure is the formal Feynman measure

$$
\mathrm{DX}_{n}:=\prod_{i, j=1}^{q_{2 n}} \prod_{\tau \in[0,1)} \mathrm{dX}_{n}(\tau)_{i j}
$$

After a simple shift of the $\mathrm{X}_{n}$ field in (5.39), one is left with a functional 
Gaussian integration in (5.51) which may be formally carried out to yield

$$
\begin{aligned}
\mathcal{Z}_{n}= & \int_{\mathcal{C}_{n}} \mathrm{DY}_{n} \frac{1}{\operatorname{det}^{\prime}\left(\frac{\beta_{2 n}}{2 g^{2}}\left(\boldsymbol{\Sigma}-\mathrm{ad}_{\mathrm{Y}_{n}}\right)\right)^{2}} \\
& +\int_{\mathcal{C}_{n}} \mathrm{DY}_{n} \int_{\operatorname{ker}\left(\boldsymbol{\Sigma}-\operatorname{ad}_{\Upsilon_{n}}\right)} \mathrm{DX}_{n} \exp \left[-\frac{\beta_{2 n}}{2 g^{2}} \int_{0}^{1} \mathrm{~d} \tau \operatorname{Tr}\left(q_{2 n} \dot{\boldsymbol{Y}}_{n}(\tau)\right.\right. \\
& \left.\left.-\left[\Xi, \mathrm{Y}_{n}(\tau)\right]\right)^{2}\right] .
\end{aligned}
$$

The prime on the determinant in (5.53) indicates that zero modes are excluded in its evaluation, while the second contributions come from the flat directions $\mathbf{X}_{n}(\tau)$ of the operators $\boldsymbol{\Sigma}-\operatorname{ad}_{Y_{n}}$ for each field configuration $\mathbf{Y}_{n}(\tau)$. We will always ignore irrelevant (infinite) constants arising from the functional integrations.

The large $n$ limit of (5.53) yields the fluctuation determinant that is intractable directly in the continuum theory [62], and our matrix model provides a systematic means of evaluating such complicated objects. Moreover, the second term can be expected to lead in the limit to the exact sum over instantons of two-dimensional noncommutative Yang-Mills theory [62]. Let us indicate how this may arise. For this, we need to study the structure of the space $\operatorname{ker}\left(\boldsymbol{\Sigma}-\operatorname{ad}_{\mathbf{Y}_{n}}\right)$, or equivalently the moduli space of solutions to the equations

$$
\boldsymbol{\Sigma} \mathrm{X}_{n}=\left[\mathrm{Y}_{n}, \mathrm{X}_{n}\right]
$$

To get a feeling for the type of solution spaces that occur, we first seek time-independent solutions of (5.54). Repeating the argument of the previous subsection, this implies that the configurations $\mathrm{X}_{n}$ are diagonal,

$$
\mathrm{X}_{n}=\sum_{i=1}^{q_{2 n}} \mathrm{i} x_{i} \mathrm{P}_{n}^{i i}
$$

with moduli $x_{i} \in \mathbb{R}$. Then (5.54) becomes

$$
\pi\left(q_{2 n}+1\right) \delta_{i j} \sum_{k=1}^{q_{2 n}} x_{k}=\mathrm{i}\left(x_{i}-x_{j}\right)\left(\mathrm{Y}_{n}\right)_{i j} .
$$

Setting $i=j$ in (5.56) yields the constraint

$$
\sum_{i=1}^{q_{2 n}} x_{i}=0
$$


For each $i \neq j$, the equations (5.56) imply that either $x_{i}=x_{j}$ or $\left(\mathrm{Y}_{n}\right)_{i j}=0$. It is straightforward to characterize the number of independent moduli in terms of the integer $r$ with $0 \leq r \leq\left(q_{2 n}-1\right) / 2$ which specifies how many nonvanishing components $\left(\mathrm{Y}_{n}\right)_{i j}, i \neq j$ there are in the given solution. If there are $r<q_{2 n}-1$ such matrix elements, then the corresponding pairs $x_{i}=x_{j}$ are equal and each eliminate one degree of freedom. From the constraint (5.57) it is straightforward to see that there are in all $q_{2 n}-r-1$ real moduli $x_{i}$. If $r \geq q_{2 n}-1$, then the constraint (5.57) eliminates all the $x_{i}$ 's. In both cases there are $q_{2 n}$ real diagonal elements and $r$ complex off-diagonal elements of the $\mathrm{Y}_{n}$ matrices. It follows that the kernel of the operator $\boldsymbol{\Sigma}-\operatorname{ad}_{\mathbf{Y}_{n}}$ admits an orthogonal decomposition into subspaces corresponding to constant $\left(\mathrm{X}_{n}, \mathrm{Y}_{n}\right)$ configurations as

$$
\operatorname{ker}\left(\boldsymbol{\Sigma}-\operatorname{ad}_{\boldsymbol{Y}_{n}}\right)=\bigoplus_{r=0}^{q_{2 n}\left(q_{2 n}-1\right) / 2} \mathcal{K}_{r}
$$

where

$$
\mathcal{K}_{r}=\left\{\begin{array}{cc}
\mathbb{R}^{q_{2 n}-r-1} \oplus \mathbb{R}^{q_{2 n}} \oplus \mathbb{C}^{r} & 0 \leq r<q_{2 n}-1 \\
\mathbb{R}^{q_{2 n}} \oplus \mathbb{C}^{r} & q_{2 n}-1 \leq r \leq q_{2 n}\left(q_{2 n}-1\right) / 2
\end{array} .\right.
$$

In the time-dependent case, we can exploit the invariance of the path integration in (5.53) under arbitrary unitary transformations of the matrices $\mathrm{X}_{n}(\tau)$ to diagonalize them. Then our analysis of the contributions to the second integral in (5.53) carries through in exactly the same manner as described above. For each of them, the path integral over the matrix trajectories $\mathrm{Y}_{n}(\tau)$ is Gaussian and yields the determinant of the operator $\frac{\beta_{2 n}}{2 g^{2}}\left(q_{2 n} \frac{\mathrm{d}}{\mathrm{d} \tau}+\operatorname{ad}_{\Xi}\right)^{2}$ on the unit circle and restricted to the subspaces of i $u\left(q_{2 n}\right)$ in which $\mathrm{Y}_{n}$ has $r$ non-zero off-diagonal matrix elements. We may evaluate (5.53) in this way to the formal expression

$$
\begin{aligned}
\mathcal{Z}_{n}= & \int_{\mathcal{C}_{n}} \mathrm{DY}_{n} \frac{1}{\operatorname{det}^{\prime}\left(\frac{\beta_{2 n}}{2 g^{2}}\left(\boldsymbol{\Sigma}-\operatorname{ad}_{Y_{n}}\right)\right)^{2}}+\sum_{r=0}^{q_{2 n}\left(q_{2 n}-1\right) / 2} \mathrm{~V}_{r} \\
& \times \sum_{i_{1}, \ldots, i_{r}=1}^{q_{2 n}} \sum_{j_{1}, \ldots, j_{r}=1}^{q_{2 n}} \prod_{\substack{k, l=1 \\
i_{k} \neq j_{l}}}^{r} \prod_{m \in \mathbb{N}_{0}}\left(\frac{2 g^{2} / \beta_{2 n}\left(q_{2 n}\right)^{2}}{m^{2}-\left(\frac{i_{k}-j_{l}}{q_{2 n}}\right)^{2}}\right)^{2},
\end{aligned}
$$

where $\mathrm{V}_{r}$ is the suitably regulated volume factor $\mathrm{V}_{r}=\operatorname{vol}\left(\mathbb{R}^{q_{2 n}-r-1}\right)$ for $0 \leq$ $r<q_{2 n}-1$, while $\mathrm{V}_{r}=1$ otherwise. It would be interesting to examine now how the appropriately regulated form of the expression (5.60) reproduces the partition sum of the corresponding continuum theory in the limit $n \rightarrow \infty$. Although we have not completely solved the problem here, the expression (5.60) once again illustrates the exact solvability of the gauge theory. 


\section{Moduli Spaces and Soliton Regularization on the Noncommutative Plane}

In this final section we shall describe the relationship between our soliton approximation of field theory on the noncommutative torus and the standard solitons on the noncommutative plane. We shall deal only with the GMS solitons which are obtained in the limit of large Moyal noncommutativity [29]. We will see that the matrix regularization of noncommutative field theory in this context provides illuminating results concerning the moduli spaces of these solitons. To help motivate the analysis, we will begin by showing how a special class of projections on the noncommutative torus naturally arise as the classical solutions of a model for the dynamics of solitons on the noncommutative torus. We will then use these projections to obtain the matrix analogs of GMS solitons, which among other things provides the starting point for the construction of one-dimensional matrix model regularizations of field theories on the noncommutative plane.

\subsection{Soliton Dynamics on the Noncommutative Torus}

We will begin by describing how to model the dynamics of projection solitons on the noncommutative torus in an adiabatic approximation. Usually, one would proceed by introducing a Kähler metric on the moduli space of fixed rank projection operators, which is typically an infinite-dimensional Grassmannian manifold. The Kähler form may be obtained as the curvature of a determinant line bundle over the Grassmannian, and with it one may construct a non-linear $\sigma$-model describing the moduli space dynamics of solitons $[50,28]$. The motion of the solitons may thereby be studied by calculating geodesics on the moduli space in the obtained Kähler metric. A non-trivial, curved geometry then corresponds to velocity dependent forces between the solitons. Here we shall instead follow the approach of [20] where non-linear $\sigma$-models in the context of noncommutative geometry were proposed. This approach exploits the inherent non-linearity of the space of projections of $\mathcal{A}_{\theta}$ directly, without explicit reference to any Kähler geometry.

We define a noncommutative field theory whose configuration space is the collection $\mathcal{P}_{\theta}$ of all projections in the algebra $\mathcal{A}_{\theta}$. The $\sigma$-model dynamics is governed by the action functional $S: \mathcal{P}_{\theta} \rightarrow \mathbb{R}^{+}$defined by

$$
S(\mathrm{P})=\frac{1}{2 \pi} f \partial_{\mu} \mathrm{P} \partial_{\mu} \mathrm{P}=\frac{1}{\pi} f \mathrm{P} \partial_{\mu} \mathrm{P} \partial_{\mu} \mathrm{P},
$$

where $\partial_{\mu}, \mu=1,2$ are the two linear derivations defined in (2.20). This 
is just the standard action that one would write down which captures the dynamics of multiple solitons (within a certain energy range), except that we utilize a flat metric in its definition. The second equality follows from the constraint $\mathrm{P}^{2}=\mathrm{P}$ and the Leibniz rule. The positivity of the trace $f$ guarantees that (6.1) is always a positive real number.

We will seek critical points of the action functional (6.1) in a given connected component of $\mathcal{P}_{\theta}$, corresponding to an equivalence class of projections of fixed rank and fixed topological charge. For this, we need to carefully take into account the non-linear structure of the space $\mathcal{P}_{\theta}$. An element $\delta \mathrm{P} \in T_{\mathrm{P}}\left(\mathcal{P}_{\theta}\right)$ in the tangent space to $\mathcal{P}_{\theta}$ at a given point $\mathrm{P}$ is not arbitrary but must fulfill two requirements. First of all, it must be Hermitian, $(\delta \mathrm{P})^{\dagger}=\delta \mathrm{P}$. Secondly, it must obey $(\mathrm{P}+\delta \mathrm{P})^{2}=\mathrm{P}+\delta \mathrm{P}+O\left((\delta \mathrm{P})^{2}\right)$, which implies that $(\mathbb{1}-\mathrm{P}) \delta \mathrm{P}=\delta \mathrm{PP}$. It follows that the most general tangent vector in $T_{\mathrm{P}}\left(\mathcal{P}_{\theta}\right)$ is of the form

$$
\delta \mathrm{P}=(\mathbb{1}-\mathrm{P}) c \mathrm{P}+\mathrm{P} c^{\dagger}(\mathbb{1}-\mathrm{P})
$$

with $c$ arbitrary elements of the algebra $\mathcal{A}_{\theta}$.

The equations of motion now follow as usual from the variational principle

$$
0=\delta S(\mathrm{P})=-\frac{1}{2 \pi} f \square(\mathrm{P}) \delta \mathrm{P},
$$

where $\square=\partial_{\mu} \partial_{\mu}$ is the Laplacian. We have used the Leibniz rule, along with invariance and cyclicity of the trace. By substituting in (6.2) and using the fact that $c \in \mathcal{A}_{\theta}$ is arbitrary, we arrive at the field equations

$$
\mathrm{P} \square(\mathrm{P})(\mathbb{1}-\mathrm{P})=0, \quad(\mathbb{1}-\mathrm{P}) \square(\mathrm{P}) \mathrm{P}=0,
$$

which together are equivalent to

$$
\mathrm{P} \square(\mathrm{P})-\square(\mathrm{P}) \mathrm{P}=0
$$

These are non-linear equations of second order which are rather difficult to solve explicitly. However, as we shall show presently, the absolute minima of the action functional (6.1) in a given connected component of $\mathcal{P}_{\theta}$ actually satisfy first order equations which are easier to solve.

For this, we recall from section 2.3 that for any projection $\mathrm{P} \in \mathcal{P}_{\theta}$, there is a topological charge (the first Chern number) defined by $(2.32)$ with $c_{1}(\mathrm{P}) \in$ $\mathbb{Z}$. Then, just as in four dimensional Yang-Mills theory, this topological quantity yields a bound on the action functional. Due to positivity of the trace $f$ and its cyclic property, we have

$$
f\left(\partial_{\mu}(\mathrm{P}) \mathrm{P} \pm \mathrm{i} \epsilon_{\mu \nu} \partial_{\nu}(\mathrm{P}) \mathrm{P}\right)^{\dagger}\left(\partial_{\mu}(\mathrm{P}) \mathrm{P} \pm \mathrm{i} \epsilon_{\mu \beta} \partial_{\beta}(\mathrm{P}) \mathrm{P}\right) \geq 0
$$


By expanding out the left-hand side of (6.6) and comparing it with (2.32) and (6.1), we then arrive at the inequality

$$
S(\mathrm{P}) \geq \pm 2 c_{1}(\mathrm{P}) \text {. }
$$

The inequality (6.7), which gives a lower bound on the action, is the analog of the one for ordinary two-dimensional $\sigma$-models [10]. From (6.6) it is clear that equality in (6.7) occurs exactly when the projection $\mathrm{P}$ satisfies the selfduality or anti-self-duality equations

$$
\left(\partial_{\mu} \mathrm{P} \pm \mathrm{i} \epsilon_{\mu \nu} \partial_{\nu} \mathrm{P}\right) \mathrm{P}=0
$$

The two equations (6.8) can be reduced to

$$
\bar{\partial}(P) P=0, \quad \partial(P) P=0,
$$

respectively; here $\partial=\frac{1}{2}\left(\partial_{1}-\mathrm{i} \partial_{2}\right)$ and $\bar{\partial}=\frac{1}{2}\left(\partial_{1}+\mathrm{i} \partial_{2}\right)$. Simple manipulations show directly that each of the equations (6.9) implies the field equations (6.5), as they should. Solutions of (6.9) are called $\sigma$-model instantons.

The non-linear equations (6.9) can be reduced to linear ones by introducing gauge degrees of freedom and by lifting them to a bundle (a module) $[20,21,22]$. The particular module is dictated by the given homotopy class that we are working in, which is in turn determined by the rank $f \mathrm{P}=p+q \theta, p, q \in \mathbb{Z}$ and topological charge $c_{1}(\mathrm{P})=q$ of the projection solutions to (6.9). We will identify the algebra $\mathcal{A}_{\theta}$ as the endomorphism algebra of a suitable bundle and regard any projection $\mathrm{P}$ as an operator on this bundle. For this, we need to consider the representation theory of another copy $\mathcal{A}_{\alpha}$ of the noncommutative torus with unitary generators $Y$ and $Z$ obeying the relation

$$
Z Y=\mathrm{e}^{2 \pi \mathrm{i} \alpha} Y Z
$$

When $\alpha$ is an irrational number, every finitely generated projective module over the algebra $\mathcal{A}_{\alpha}$ which is not free is isomorphic to a Heisenberg module. As these modules will also be of central importance in the following, we shall review their basic properties here [18].

As already mentioned, any such module $\mathcal{E}_{p, q}$ is characterized by two integers $p, q$ satisfying $p+q \alpha>0$, which can be taken to be relatively prime with $q>0$, or $p=0$ and $q=1$. As a vector space, the module

$$
\mathcal{E}_{p, q}=S(\mathbb{R}) \otimes \mathbb{C}^{q}
$$

is the space of Schwartz functions of one continuous variable $s \in \mathbb{R}$ and one discrete variable $k \in \mathbb{Z}_{q}$. By introducing the notation

$$
\varepsilon=p / q-\alpha
$$


the space (6.11) is made into a right module over $\mathcal{A}_{\alpha}$ by defining

$$
\begin{aligned}
(\xi Y)_{k}(s) & :=\xi_{[k-p]_{q}}(s-\varepsilon), \\
(\xi Z)_{k}(s) & :=\mathrm{e}^{2 \pi \mathrm{i}(s-k / q)} \xi_{k}(s)
\end{aligned}
$$

for $\xi \in \mathcal{E}_{p, q}$, with the relations (6.10) being easily verified. On the module (6.11) one defines an $\mathcal{A}_{\alpha}$-valued Hermitian structure

$$
\langle\cdot, \cdot\rangle_{\alpha}: \mathcal{E}_{p, q} \times \mathcal{E}_{p, q} \longrightarrow \mathcal{A}_{\alpha}
$$

by the formula

$$
\langle\xi, \eta\rangle_{\alpha}:=\sum_{(m, r) \in \mathbb{Z}^{2}} \sum_{k=0}^{q-1} \int_{\mathbb{R}} \mathrm{d} s \overline{\xi_{[k-m p]_{q}}(s-m \varepsilon)} \eta_{k}(s) \mathrm{e}^{-2 \pi \mathrm{i} r(s-k / q)} Y^{m} Z^{r}
$$

for $\xi, \eta \in \mathcal{E}_{p, q}$. Note the antilinearity of the first factor.

The endomorphism algebra $\operatorname{End}_{\mathcal{A}_{\alpha}}\left(\mathcal{E}_{p, q}\right)$, which acts from the left on $\mathcal{E}_{p, q}$, can be identified with the original copy $\mathcal{A}_{\theta}$ of the noncommutative torus where the noncommutativity parameter $\theta$ is "uniquely" determined by $\alpha$ in the following way. Since $p$ and $q$ are relatively prime, there exist integers $a, b \in \mathbb{Z}$ such that $b q-a p=1$. Then the noncommutativity parameter is given by the discrete Möbius transformation

$$
\theta=\frac{a \alpha-b}{q \alpha-p}
$$

Notice that given any other pair of integers $a^{\prime}, b^{\prime} \in \mathbb{Z}$ with $b^{\prime} q-a^{\prime} p=1$, one has $\theta^{\prime}-\theta \in \mathbb{Z}$ so that $\mathcal{A}_{\theta^{\prime}} \cong \mathcal{A}_{\theta}$. It follows that the algebra $\operatorname{End}_{\mathcal{A}_{\alpha}}\left(\mathcal{E}_{p, q}\right)$ is generated by the two unitary operators $U$ and $V$ which act from the left on $\mathcal{E}_{p, q}$ by

$$
\begin{aligned}
(U \xi)_{k}(s) & :=\xi_{[k-1]_{q}}(s-1 / q), \\
(V \xi)_{k}(s) & :=\mathrm{e}^{\frac{2 \pi \mathrm{i}}{q}(s / \varepsilon+a k)} \xi_{k}(s),
\end{aligned}
$$

and one easily verifies the defining relations (2.3) of the algebra $\mathcal{A}_{\theta}$.

On $\mathcal{E}_{p, q}$ there is also an $\mathcal{A}_{\theta}$-valued inner product

$$
\langle\cdot, \cdot\rangle_{\theta}: \mathcal{E}_{p, q} \times \mathcal{E}_{p, q} \longrightarrow \mathcal{A}_{\theta}
$$

which is given explicitly by

$$
\begin{aligned}
\langle\xi, \eta\rangle_{\theta}:= & \frac{1}{|q \varepsilon|} \sum_{(m, r) \in \mathbb{Z}^{2}} \sum_{k=0}^{q-1} \int_{\mathbb{R}} \mathrm{d} s \xi_{k}(s) \overline{\eta_{[k-m]_{q}}(s-m / q)} \\
& \times \mathrm{e}^{-\frac{2 \pi \mathrm{i}}{q \varepsilon}(s-m / q+a k \varepsilon)} U^{m} V^{r}
\end{aligned}
$$


for $\xi, \eta \in \mathcal{E}_{p, q}$. Notice that now the antilinearity is in the second factor. The key feature of this Hermitian structure is that it is compatible with the $\mathcal{A}_{\alpha}$-valued one $(6.14,6.15)$,

$$
\langle\xi, \eta\rangle_{\theta} \zeta=\xi\langle\eta, \zeta\rangle_{\alpha}
$$

for all $\xi, \eta, \zeta \in \mathcal{E}_{p, q}$. This means that the $\mathcal{A}_{\theta^{-}} \mathcal{A}_{\alpha}$ bimodule $\mathcal{E}_{p, q}$ provides a Morita equivalence between the two algebras $\mathcal{A}_{\theta}$ and $\mathcal{A}_{\alpha}$. Physically, the compatibility condition (6.20) corresponds to T-duality between the vertex operator algebras of $\left(p^{\prime}-2\right)-\left(p^{\prime}-2\right)$ and $p^{\prime}-p^{\prime}$ strings acting on the Hilbert space of $p^{\prime}$ - $\left(p^{\prime}-2\right)$ open string states in the low-energy limit [45, 72]. These open string modes stretch between a single $\mathrm{D}\left(p^{\prime}-2\right)$-brane and a collection of $p$ coincident $\mathrm{D} p^{\prime}$-branes carrying $q$ units of vortex $\mathrm{D}\left(p^{\prime}-2\right)$-brane charge.

Using the previous construction one can now build projections on the algebra $\mathcal{A}_{\theta}$ by picking suitable elements $\xi^{\prime} \in \mathcal{E}_{p, q}$ with $\left\langle\xi^{\prime}, \xi^{\prime}\right\rangle_{\alpha}=\mathbb{1}$. The bimodule property (6.20) then implies that $\mathrm{P}=\left\langle\xi^{\prime}, \xi^{\prime}\right\rangle_{\theta}$ is a non-trivial projection in $\mathcal{A}_{\theta}$. Furthermore, by using the identification $\mathcal{A}_{\theta} \cong \operatorname{End}_{\mathcal{A}_{\alpha}}\left(\mathcal{E}_{p, q}\right)$, any such a projection may be equivalently written in the more suggestive form

$$
\mathrm{P}=\left\langle\xi\left(\langle\xi, \xi\rangle_{\alpha}\right)^{-1 / 2}, \xi\left(\langle\xi, \xi\rangle_{\alpha}\right)^{-1 / 2}\right\rangle_{\theta}=|\xi\rangle \frac{\mathbb{1}}{\langle\xi, \xi\rangle_{\alpha}}\langle\xi|
$$

where for each element $|\xi\rangle \in \mathcal{E}_{p, q}$ the corresponding dual element $\langle\xi| \in\left(\mathcal{E}_{p, q}\right)^{*}$ is defined by means of the Hermitian structure as $\langle\xi|(\eta)=\langle\xi, \eta\rangle_{\alpha} \in \mathcal{A}_{\alpha}$, and we have only assumed now that $\langle\xi, \xi\rangle_{\alpha}$ is an invertible element of the algebra $\mathcal{A}_{\alpha}$.

In order to translate the self-duality equations (6.9) for $\mathrm{P}$ to equations for $\xi$, we need to introduce a gauge connection on the right $\mathcal{A}_{\alpha}$-module $\mathcal{E}_{p, q}$. This is done by means of two covariant derivatives ${ }^{7} \nabla_{1}, \nabla_{2}: \mathcal{E}_{p, q} \rightarrow \mathcal{E}_{p, q}$ which are given explicitly by

$$
\left(\nabla_{1} \xi\right)_{k}(s):=\frac{2 \pi \mathrm{i}}{\varepsilon} s \xi_{k}(s), \quad\left(\nabla_{2} \xi\right)_{k}(s):=\frac{\mathrm{d} \xi_{k}(s)}{\mathrm{d} s} .
$$

Notice that the discrete index $k$ is not touched. This connection has constant curvature

$$
\left[\nabla_{1}, \nabla_{2}\right]=-\frac{2 \pi \mathrm{i}}{\varepsilon} \mathbb{1}
$$

and the two operators (6.22) satisfy a Leibniz rule with respect to the right action,

$$
\nabla_{\mu}(\xi a)=\left(\nabla_{\mu} \xi\right) a+\xi\left(\partial_{\mu} a\right), \quad \mu=1,2,
$$

\footnotetext{
${ }^{7}$ The covariant derivative $\nabla$ introduced here should not be confused with the approximate derivation $\nabla$ introduced in section 4.2 .
} 
for any $\xi \in \mathcal{E}_{p, q}$ and $a \in \mathcal{A}_{\alpha}$. They are also compatible with the $\mathcal{A}_{\alpha}$-valued Hermitian structure,

$$
\partial_{\mu}\langle\xi, \eta\rangle_{\alpha}=\left\langle\nabla_{\mu} \xi, \eta\right\rangle_{\alpha}+\left\langle\xi, \nabla_{\mu} \eta\right\rangle_{\alpha}, \quad \mu=1,2,
$$

for any $\xi, \eta \in \mathcal{E}_{p, q}$. Furthermore, by using compatibility (6.25) and the right Leibniz rule (6.24) one can show that the induced derivations on the endomorphism algebra,

$$
\delta_{1}, \delta_{2}: \operatorname{End}_{\mathcal{A}_{\alpha}}\left(\mathcal{E}_{p, q}\right) \longrightarrow \operatorname{End}_{\mathcal{A}_{\alpha}}\left(\mathcal{E}_{p, q}\right), \quad \delta_{\mu}(T):=\left[\nabla_{\mu}, T\right]
$$

are proportional to the generators of the infinitesimal action of the commutative torus $\mathbb{T}^{2}$ on $\mathcal{A}_{\theta} \cong \operatorname{End}_{\mathcal{A}_{\alpha}}\left(\mathcal{E}_{p, q}\right)$,

$$
\delta_{\mu}=\frac{1}{q \varepsilon} \partial_{\mu} .
$$

It is because of this property that we select the particular connection (6.22).

Then, by using these ingredients, it is straightforward to show that the projection $\mathrm{P}$ in (6.21) satisfies the self-duality equations (6.9) if and only if there exists an element $\rho \in \mathcal{A}_{\alpha}$ such that

$$
\bar{\nabla} \xi=\xi \rho,
$$

with $\bar{\nabla}=\frac{1}{2}\left(\nabla_{1}+\mathrm{i} \nabla_{2}\right)$. This equation follows from a simple computation with the element $\rho=\left(\langle\xi, \xi\rangle_{\alpha}\right)^{-1}\langle\xi, \bar{\nabla} \xi\rangle_{\alpha}$. When $\rho$ is constant (i.e. it is proportional to the unit of $\mathcal{A}_{\alpha}, \rho=\lambda \mathbb{1}$ with $\lambda \in \mathbb{C}$ ), the equation (6.28) reduces to the simple differential equation

$$
\frac{\mathrm{d} \xi}{\mathrm{d} s}+\left(\frac{2 \pi s}{\varepsilon}+2 \mathrm{i} \lambda\right) \xi=0
$$

whose solutions are the Gaussian fields

$$
\xi_{\lambda}(s)=\boldsymbol{A} \mathrm{e}^{-\pi s^{2} / \varepsilon-2 \mathrm{i} \lambda s} .
$$

The vector $\boldsymbol{A}=\left(A_{1}, \ldots, A_{q}\right) \in \mathbb{C}^{q}$ can be taken to lie in the complex projective space $\mathbb{C P}^{q-1}$ by removing an inessential normalization. It is possible to prove that, for all values of the deformation parameter $\theta$, the norms $\left\langle\xi_{\lambda}, \xi_{\lambda}\right\rangle_{\alpha} \in \mathcal{A}_{\alpha}$ are invertible [14, 76]. Accordingly, the Gaussian functions (6.30) provide a complex one-parameter family of solutions $\mathrm{P}_{\lambda}=$ $\left|\xi_{\lambda}\right\rangle\left(\left\langle\xi_{\lambda}, \xi_{\lambda}\right\rangle_{\alpha}\right)^{-1}\left\langle\xi_{\lambda}\right|$ of the self-duality equations (6.9). The projection $\mathrm{P}_{\lambda} \in$ $\mathcal{A}_{\theta}$ has rank $f \mathrm{P}_{\lambda}=p+q \theta$ and topological charge $c_{1}\left(\mathrm{P}_{\lambda}\right)=q$.

The physically relevant values of the complex parameter $\lambda$ can be restricted by gauge symmetry. Any two elements $\xi$ and $\xi^{\prime}$ of $\mathcal{E}_{p, q}$ provide 
different projections (6.21) if and only if they belong to different orbits of the action of the group $G L\left(\mathcal{A}_{\alpha}\right)$ of invertible elements of $\mathcal{A}_{\alpha}$ which acts on the right on $\mathcal{E}_{p, q}$,

$$
|\xi\rangle \longmapsto\left|\xi^{g}\right\rangle=|\xi\rangle g, \quad g \in G L\left(\mathcal{A}_{\alpha}\right) .
$$

Note that we do not require $g$ to be unitary. The action (6.31) preserves the invertibility of $\langle\xi, \xi\rangle_{\alpha}$ and leaves the corresponding projection (6.21) invariant. Furthermore, from the Leibniz rule for the gauge connection it follows that if $\xi$ is a solution of the self-duality equation (6.28), then the transformed vector $\xi^{g}$ also solves an equation of the form (6.28), $\bar{\nabla} \xi^{g}=\xi^{g} \rho^{g}$, with the element $\rho \in \mathcal{A}_{\alpha}$ modified to

$$
\rho \longmapsto \rho^{g}=g^{-1} \rho g+g^{-1} \bar{\partial} g .
$$

Elements of the group $G L\left(\mathcal{A}_{\alpha}\right)$ thereby play the role of complex gauge transformations.

In the case of the Gaussian fields (6.30), it is straightforward to show from (6.32) with $\rho=\lambda \mathbb{1}$ and $\rho^{g}=\lambda^{\prime} \mathbb{1}$ that $\xi_{\lambda}$ and $\xi_{\lambda^{\prime}}$ are gauge equivalent if and only if $\xi_{\lambda^{\prime}}=\xi_{\lambda} U^{m} V^{r}$ for some pair of integers $(m, r) \in \mathbb{Z}^{2}$. The parameters of the Gaussian functions are then related by

$$
\lambda^{\prime}=\lambda+\pi \mathrm{i}(m+\mathrm{i} r) .
$$

It follows that the gauge inequivalent parameters $\lambda$ make up an ordinary torus $\mathbb{T}^{2}$. As we will see later on, the moduli $\lambda \in \mathbb{T}^{2}$ correspond to the locations of the solitons on the underlying torus. The moduli space of Gaussian fields (6.30) is thus $\mathbb{C P}^{q-1} \times \mathbb{T}^{2}$. For further aspects of these and other constructions, see $[20,21,22]$.

\subsection{The Boca Projection}

Let us now describe explicitly a particular instance of the globally minimizing soliton projections of the previous subsection; it will play an important role in the following. The Boca projection on the noncommutative torus comes from choosing the simplest bimodule $\mathcal{E}_{0,1}=S(\mathbb{R})$, for which $\varepsilon=1 / \theta, a=p=0$, $b=q=1$, and $\alpha=-1 / \theta$ in the above construction. With the Gaussian Schwartz function

$$
\xi(s)=\xi_{\lambda=0}(s)=\mathrm{e}^{-\pi \theta s^{2}},
$$

which is such that the element $\langle\xi, \xi\rangle_{-1 / \theta} \in \mathcal{A}_{-1 / \theta}$ is invertible $[14,76]$, it follows that

$$
\mathrm{B}_{\theta}:=\left\langle\xi\left(\langle\xi, \xi\rangle_{-1 / \theta}\right)^{-1 / 2}, \xi\left(\langle\xi, \xi\rangle_{-1 / \theta}\right)^{-1 / 2}\right\rangle_{\theta}
$$


is a projection on $\mathcal{A}_{\theta}$ which is homotopic to the Powers-Rieffel projection (2.28).

The general form of the Boca projection (6.35) may be deduced by using the $\mathcal{A}_{-1 / \theta^{-} \text {action }(6.13)}$ on (6.34) and the inner product (6.15) to explicitly compute the element $\langle\xi, \xi\rangle_{-1 / \theta} \in \mathcal{A}_{-1 / \theta}$. The square root may be computed by using holomorphic functional calculus, and one thereby finds [56]

$$
\begin{aligned}
\xi(s)\left(\langle\xi, \xi\rangle_{-1 / \theta}\right)^{-1 / 2}= & \mathrm{e}^{-\pi s^{2} / \theta}\left[1-\sum_{k \in \mathbb{N}} \frac{(2 k-3) ! !}{k !}\left(\frac{\theta}{8}\right)^{k / 2}\right. \\
& \left.\times \sum_{\substack{(\boldsymbol{m}, \boldsymbol{r}) \in \mathbb{Z}^{k} \times \mathbb{Z}^{k} \\
(\boldsymbol{m}, \boldsymbol{r}) \neq(\mathbf{0}, \mathbf{0})}} \mathrm{e}^{-Q_{k}(\boldsymbol{m}, \boldsymbol{r})} \prod_{i=1}^{k} \mathrm{e}^{-(2 \pi s / \theta)\left(m_{i}+\mathrm{i} r_{i}\right)}\right],
\end{aligned}
$$

with the convention $(-1) ! !:=1$ and $Q_{k}$ the quadratic form on $\mathbb{Z}^{k} \times \mathbb{Z}^{k}$ defined by

$$
\begin{aligned}
Q_{k}(\boldsymbol{m}, \boldsymbol{r})= & \frac{\pi}{2 \theta} \sum_{i=1}^{k}\left[\left(m_{i}\right)^{2}+\left(r_{i}\right)^{2}\right]+\frac{\pi}{\theta}\left(\sum_{i=1}^{k} m_{i}\right)^{2} \\
& +\frac{\pi \mathrm{i}}{\theta}\left(\sum_{i=1}^{k} m_{i} r_{i}+2 \sum_{i<j} m_{i} r_{j}\right) .
\end{aligned}
$$

The corresponding projection is given by (6.35) and (6.19), and it will be used in the following to relate the soliton basis of Section 3 to matrix noncommutative solitons on $\mathbb{R}^{2}$.

At the special rational values $\theta=1 / k, k \in \mathbb{N}$ (in which case the algebra $\mathcal{A}_{-1 / \theta} \cong C^{\infty}\left(\mathbb{T}^{2}\right)$ is commutative), the Boca projection can be expressed in terms of theta-functions of the generators $U$ and $V$ of $\mathcal{A}_{\theta}$. For this, we introduce the elliptic Jacobi-Erderlyi theta-functions

$$
\vartheta_{\frac{a}{N}, b}(\nu \mid \mathrm{i} \sigma)=\sum_{m \in \mathbb{Z}} \mathrm{e}^{-\pi \sigma(m+a / N)^{2}+2 \pi \mathrm{i}(m+a / N) b} \mathrm{e}^{2 \pi \mathrm{i}(N m+a) \nu}
$$

for $a \in \mathbb{Z}, b \in \mathbb{R}$ and $N \in \mathbb{N}$, which are holomorphic in $\nu \in \mathbb{C}$ for moduli $\sigma \in \mathbb{C}$ with $\operatorname{Re}(\sigma)>0$. We use the convention that $N=1$ when $a=0$ in (6.38). Then by taking $k \in \mathbb{N}$ even for definiteness, and using the Weyl maps $\rho$ and $\rho^{\prime}$ of (2.27) and (2.40), the Boca projection (6.35) may be expressed 
succinctly as [14]

$$
\begin{aligned}
\mathrm{B}_{1 / k}= & \frac{1}{k \rho\left(\vartheta_{0,0}\left(x^{k} \mid \frac{\mathrm{i} k}{2}\right)\right) \rho^{\prime}\left(\vartheta_{0,0}\left(y^{k} \mid \frac{\mathrm{i} k}{2}\right)\right)} \\
& \times \sum_{l, m=0}^{k-1} \mathrm{e}^{-\pi \mathrm{i} l m / k} \rho\left(\vartheta_{\frac{m}{k}, \frac{l}{2}}\left(x \mid \frac{\mathrm{i} k}{2}\right)\right) \rho^{\prime}\left(\vartheta_{\frac{l}{k}, \frac{m}{2}}\left(y \mid \frac{\mathrm{i} k}{2}\right)\right) .
\end{aligned}
$$

The Wigner function on $\mathbb{T}^{2}$ corresponding to the projection (6.39), which is real and exhibits localized bump configurations, is displayed in [28]. This form will be used later on to give a physical interpretation to the relationship between torus solitons and solitons on the noncommutative plane.

\subsection{GMS Soliton Expansions}

Let us now consider the noncommutative plane $\mathbb{R}_{\Theta}^{2}$, which is defined heuristically by the Heisenberg commutation relation $[y, x]=2 \mathrm{i} \Theta$. We will assume that $\Theta>0$ for definiteness. The algebra $C^{\infty}\left(\mathbb{R}_{\Theta}^{2}\right)$ may be identified as the appropriate completion of the polynomial algebra $F\left(\mathbb{R}^{2}\right) / I_{\Theta}$, where $F\left(\mathbb{R}^{2}\right)=\mathbb{C}\langle\mathbb{1}, x, y\rangle$ is the free unital algebra on two generators $x, y$, and $I_{\Theta}$ is the two-sided ideal of $F\left(\mathbb{R}^{2}\right)$ generated by the element $y x-x y-2 \mathrm{i} \Theta \mathbb{1}$. As a Heisenberg algebra, it has a unique irreducible representation which is the usual Fock space

$$
\mathcal{F}=\ell^{2}\left(\mathbb{N}_{0}\right)=\overline{\operatorname{span}_{\mathbb{C}}}\left\{|m\rangle \mid m \in \mathbb{N}_{0}\right\}
$$

for the Schrödinger representation of quantum mechanics. In (6.40), the vectors $|m\rangle$ are the elements of the usual orthonormal number basis of a onedimensional harmonic oscillator, $\langle m \mid n\rangle=\delta_{m n}$, and the appropriate completion is indicated which will always be implicitly understood in what follows. In particular, a basis for the algebra of bounded linear operators on $\mathcal{F}$ is provided by the set $\left\{|n\rangle\left\langle m|| m, n \in \mathbb{N}_{0}\right\}\right.$.

The Weyl map $\Omega$ and star-product on the noncommutative plane are defined analogously to (2.5), (2.6) and (2.9) by using Fourier transformation of fields on $\mathbb{R}^{2}[75]$. In particular, the Wigner functions on $\mathbb{R}^{2}$ corresponding to the rank one Fock space operators $|n\rangle\langle m|$ are the Landau wavefunctions

$$
\psi_{n, m}(w, \bar{w})=\frac{1}{\sqrt{4 \pi \Theta}} \Omega^{-1}(|n\rangle\langle m|),
$$

where $w, \bar{w}$ are complex coordinates on the plane. They are the orthonormal eigenfunctions in $L^{2}\left(\mathbb{R}^{2}\right)$ of the Landau Hamiltonian for a charged particle 
moving on $\mathbb{R}^{2}$ under the influence of a constant, perpendicularly applied magnetic field $B=\Theta^{-1}$. The ground state wavefunction is the Gaussian field

$$
\psi_{0,0}(w, \bar{w})=\frac{1}{\sqrt{\pi \Theta}} \mathrm{e}^{-|w|^{2} / 2 \Theta},
$$

while the higher Landau levels can be obtained from (6.42) by application of the differential creation operators

$$
a^{\dagger}:=\frac{1}{2}\left(-\sqrt{\Theta} \frac{\partial}{\partial \bar{w}}+\frac{w}{\sqrt{\Theta}}\right), \quad b^{\dagger}:=\frac{1}{2}\left(-\sqrt{\Theta} \frac{\partial}{\partial w}+\frac{\bar{w}}{\sqrt{\Theta}}\right)
$$

as

$$
\begin{aligned}
\psi_{n, m}(w, \bar{w})= & \frac{\left(a^{\dagger}\right)^{n}}{\sqrt{n !}} \frac{\left(b^{\dagger}\right)^{m}}{\sqrt{m !}} \psi_{0,0}(w, \bar{w}) \\
= & \frac{(-1)^{\min (n, m)}}{\max (n, m) !} \sqrt{\frac{n ! m !}{\pi \Theta}}\left(\frac{|w|^{2}}{\Theta}\right)^{|n-m| / 2} \mathrm{e}^{\mathrm{i}(n-m) \arg (w)} \\
& \times \mathrm{e}^{-|w|^{2} / 2 \Theta} L_{\min (n, m)}^{|n-m|}\left(|w|^{2} / \Theta\right),
\end{aligned}
$$

where

$$
L_{k}^{r}(t)=\sum_{l=0}^{k}(-1)^{l}\left(\begin{array}{l}
k+r \\
k-l
\end{array}\right) \frac{t^{l}}{l !}
$$

are the associated Laguerre polynomials.

From the Wigner representation (6.41) it follows immediately that these functions obey the star-product projection relation

$$
\psi_{n, m} \star \psi_{n^{\prime}, m^{\prime}}=\frac{1}{\sqrt{4 \pi \Theta}} \delta_{m n^{\prime}} \psi_{n, m^{\prime}},
$$

and thereby determine solitonic configurations of noncommutative field theory on $\mathbb{R}^{2}[29]$. The basic Gaussian soliton (6.42) can be centered about any point on the plane by using the exact translational symmetry of noncommutative field theory, and hence the one-soliton moduli space as a complex manifold is isomorphic to $\mathbb{C}$. The Wigner function of the rank $k$ projection

$$
\mathrm{P}_{(k)}=\sum_{m=0}^{k-1}|m\rangle\langle m|
$$

describes $k$ solitons, and the corresponding moduli space is the $k^{\text {th }}$ symmetric product $\mathbb{C}^{k} / S_{k}$ of the single soliton moduli space, endowed with a smooth Kähler metric [28]. The basic Murray-von Neumann partial isometry is provided by the shift operator

$$
\mathcal{S}_{\infty}=\sum_{m \in \mathbb{N}_{0}}|m+1\rangle\langle m|
$$


with $\left(\mathcal{S}_{\infty}^{\dagger}\right)^{k}\left(\mathcal{S}_{\infty}\right)^{k}=\mathbb{1}$ and $\left(\mathcal{S}_{\infty}\right)^{k}\left(\mathcal{S}_{\infty}^{\dagger}\right)^{k}=\mathbb{1}-\mathrm{P}_{(k)}$. Again the moduli space of partial isometries $\left(\mathcal{S}_{\infty}\right)^{k}$, and hence the moduli space of $k$ D-branes on $\mathbb{R}^{2}$, is manifestly $\mathbb{C}^{k} / S_{k}$.

The key feature of the Landau wavefunctions within the present context is that they are complete in $L^{2}\left(\mathbb{R}^{2}\right)$, so that any field $f \in C^{\infty}\left(\mathbb{R}^{2}\right)$ may be expanded as

$$
f(w, \bar{w})=\sum_{(n, m) \in \mathbb{N}_{0}^{2}} f_{n, m} \psi_{n, m}(w, \bar{w})
$$

where the expansion coefficients $\left\{f_{n, m}\right\} \in S\left(\mathbb{Z}^{2}\right)$ are chosen to yield finite Landau semi-norms

$$
\|f\|_{\mathrm{L}, k}:=\left(\sum_{(n, m) \in \mathbb{N}_{0}^{2}} \Theta^{2 k}(2 n+1)^{k}(2 m+1)^{k}\left|f_{n, m}\right|^{2}\right)^{1 / 2}<\infty \quad \forall k \in \mathbb{N}_{0}
$$

This suggests a natural regularization of noncommutative fields on $\mathbb{R}^{2}$ in which the Landau quantum numbers are truncated to a finite range $0 \leq$ $n, m \leq N-1$, and the expansion coefficients of (6.49) are assembled into an $N \times N$ matrix $\left(f_{n, m}\right) \in \mathbb{M}_{N}(\mathbb{C})$ [49]. Similar truncations have also been used as approximations of a disc [52], a strip [7] and a punctured plane [67]. Because of (6.46), the star-product $f \star f^{\prime}$ of two fields corresponds to the usual matrix product of $\left(f_{n, m}\right)$ and $\left(f_{n, m}^{\prime}\right)$ in $\mathbb{M}_{N}(\mathbb{C})[30]$, and the noncommutativity of the plane $\mathbb{R}_{\Theta}^{2}$ is thereby mapped into the noncommutativity of matrix multiplication. In addition, by orthonormality, spacetime integrals over $\mathbb{R}^{2}$ of fields $f$ are given by traces of their matrices $\left(f_{n, m}\right)$. Thus the expansion of functions in the GMS soliton basis provides a very natural way to map noncommutative field theory on $\mathbb{R}^{2}$ onto a zero-dimensional matrix model [49]. The regularization provided by the finite matrix dimension $N$ controls both ultraviolet and infrared divergences at the same time [48] and avoids the renormalization problems set in by UV/IR mixing. The limit $N \rightarrow \infty$ required to map back onto the original continuum field theory corresponds to the usual 't Hooft planar limit [54, 49]. However, as mentioned earlier, this is a subtle point, as the algebra of the noncommutative plane is not the inductive limit of finite-dimensional algebras, and so one has to define this limit carefully as in [46]. Moreover, in general this limit will not commute with the scaling limits used in ordinary field theoretic renormalization [49]. In the following we will relate the GMS soliton basis to that of the previous sections and show how to regulate field theories on noncommutative $\mathbb{R}^{2}$ by means of one-dimensional matrix models. 


\subsection{From Torus Solitons to GMS Solitons}

To relate the GMS soliton regularization above to that of the previous sections, we shall first describe how to pass from solitons on the noncommutative torus to the noncommutative solitons (6.44) on $\mathbb{R}^{2}$ [43]. For this, we consider the Boca projection (6.35) in the limit $\theta \rightarrow 0$. In that limit, the Schwartz function (6.36) reduces to

$$
\xi(s)\left(\langle\xi, \xi\rangle_{-1 / \theta}\right)^{-1 / 2}=\left(\frac{2}{\theta}\right)^{1 / 4} \mathrm{e}^{-\pi s^{2} / \theta}+O\left(\mathrm{e}^{-2 \pi s^{2} / \theta}\right) .
$$

By substituting (6.51) into (6.35) and performing the resulting Gaussian integrals over $s$ in (6.19) we arrive at the Boca projection in this limit as

$$
\psi_{\theta}:=\lim _{\theta \rightarrow 0} \mathrm{~B}_{\theta}=\theta \sum_{(m, r) \in \mathbb{Z}^{2}} \mathrm{e}^{-\frac{\pi \theta}{2}\left(m^{2}-2 \mathrm{i} m r+r^{2}\right)} U^{m} V^{r} .
$$

By using the map (2.6) it follows that the Wigner function corresponding to the element (6.52) of $\mathcal{A}_{\theta}$ decouples the sums over $m$ and $r$. It thereby reduces to a product of elliptic functions of the form

$$
\Omega^{-1}\left(\boldsymbol{\psi}_{\theta}\right)(x, y)=\theta \vartheta_{0,0}\left(x \mid \frac{\mathrm{i} \theta}{2}\right) \vartheta_{0,0}\left(y \mid \frac{\mathrm{i} \theta}{2}\right) .
$$

The function (6.53) is plotted in Fig. 4. In contrast to the projections used earlier, this soliton configuration resembles the Gaussian GMS soliton (6.42). In particular, for any $\theta$ its height is always 2 , and as $\theta$ decreases its width becomes smaller and a spike develops. In the limit $\theta=0$, the function (6.53) vanishes everywhere except at the origin of $\mathbb{R}^{2}$ where it is finite. However, this limit is not smooth, and for $\theta=0$ the soliton does not exist, as there are no non-trivial projections in a commutative algebra. tion)

By using the Jacobi inversion formula (equivalently Poisson resumma-

$$
\vartheta_{0,0}(\nu \mid \mathrm{i} \sigma)=\frac{1}{\sqrt{\sigma}} \mathrm{e}^{-\pi \nu^{2} / \sigma} \vartheta_{0,0}\left(\frac{\mathrm{i} \nu}{\sigma} \mid \frac{\mathrm{i}}{\sigma}\right),
$$

the $\theta \rightarrow 0$ limit will pick out the $m=0$ mode of the corresponding thetafunctions in (6.53). This yields

$$
\Omega^{-1}\left(\boldsymbol{\psi}_{\theta}\right)(x, y)=2 \mathrm{e}^{-2 \pi\left(x^{2}+y^{2}\right) / \theta} .
$$

We now map the local coordinates $(x, y)$ of the torus $\mathbb{T}^{2}$ onto those $(w, \bar{w})$ of its universal covering space $\mathbb{R}^{2}$ by rescaling the cycles of $\mathbb{T}^{2}$ to give them a radius $R$ and then taking the decompactification limit $R \rightarrow \infty$. This relates the two sets of coordinates as

$$
(x+\mathrm{i} y, x-\mathrm{i} y)=\frac{(w, \bar{w})}{2 \pi R}
$$



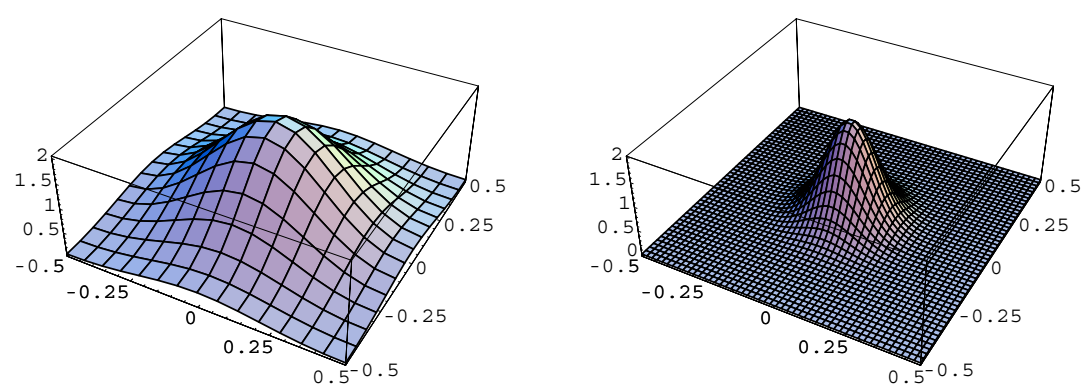

Figure 4: The soliton field configuration corresponding to the Boca projection in the small $\theta$ limit. Axes are as in Fig. 2. Displayed are its shapes when the noncommutativity parameter is taken to be the inverse of the golden mean (left) and for $\theta=\frac{1}{10}$ (right).

which implies that the noncommutativity parameters are related through

$$
\theta=\frac{\Theta}{\pi R^{2}},
$$

consistently with the small $\theta$ limit used above.

It follows that in the large area limit of the toroidal theory, with the noncommutativity parameter $\Theta$ of $\mathbb{R}^{2}$ held fixed, the Wigner function (6.55) coincides with the basic Gaussian GMS soliton (6.42),

$$
\Omega^{-1}\left(\boldsymbol{\psi}_{\theta}\right)(w, \bar{w})=\sqrt{4 \pi \Theta} \psi_{0,0}(w, \bar{w}) .
$$

Equivalently, by using (6.41) one may identify the operators

$$
\boldsymbol{\psi}_{\theta}=|0\rangle\langle 0|
$$

in the decompactification limit of the torus. The higher Landau levels $\psi_{n, m}$ (or equivalently $|n\rangle\langle m|$ ) can be similarly obtained by using higher rank projections on the noncommutative torus, but we will content ourselves here with the fact that they can obtained from (6.58) through the identity (6.44).

A nice physical interpretation of this relationship between the toroidal and planar noncommutative solitons may be given in the case of rational $\theta=1 / k$, with $k \in \mathbb{N}$ even, as used in arriving at (6.39). For this, we note that the Landau wavefunction (6.42) can be multiplied by an arbitrary antiholomorphic function $\phi(\bar{w})$ on $\mathbb{C}$, which we may choose so as to give the state a momentum $p / 2 \Theta$ along the $x$ direction of $\mathbb{R}^{2}$ and hence write

$\psi_{0,0}(w, \bar{w} ; p):=\phi_{p}(\bar{w}) \psi_{0,0}(w, \bar{w})=\frac{1}{\sqrt{\pi \Theta}} \mathrm{e}^{-\left(2 p^{2}-4 \mathrm{i} p \bar{w}-\bar{w}^{2}\right) / 2 \Theta} \mathrm{e}^{-|w|^{2} / 2 \Theta}$. 
We will assume that the area of the torus $\mathbb{T}^{2}$ is quantized such that the quantity $\frac{8}{R^{2}}$ is an integer; for definiteness we choose $R^{2}=8$.

We can map the wavefunction (6.60) to one on the torus by regarding a soliton on the torus as an infinite lattice of solitons on the plane. Taking the quotient of $\mathbb{R}^{2}$ by the momentum lattice $\mathbb{Z}^{2}$ leads, using (6.57), to a quantization condition $p=4 \pi m / k, m \in \mathbb{Z}$ on the momentum in the $x$ direction. On the other hand, $p$ is also the $y$ coordinate of the location of the soliton (6.60) on $\mathbb{R}^{2}$, and so for each quantum number $m$ it can assume $\frac{k}{2}$ different values $p=\frac{4 \pi r}{k}+2 \pi m, r=1, \ldots, \frac{k}{2}$. By summing over all $m \in \mathbb{Z}$ to take the quotient, we arrive from (6.60) at $\frac{k}{2}$ basic Landau wavefunctions on the torus. Using (6.56) they may be written as

$$
\begin{aligned}
\psi_{0,0}(x, y ; r)= & \sum_{m \in \mathbb{Z}} \psi_{0,0}(w, \bar{w} ; 2 \pi m+4 \pi r / k) \\
= & \frac{1}{\sqrt{8 \pi^{2} \theta}} \mathrm{e}^{2 \pi k(x-\mathrm{i} y)^{2}} \mathrm{e}^{-2 \pi k\left(x^{2}+y^{2}\right)} \\
& \times \vartheta_{\frac{2 r}{k}, 0}\left(\sqrt{2} k(x-\mathrm{i} y) \mid \frac{\mathrm{i} k}{2}\right)
\end{aligned}
$$

with $r=1, \ldots, \frac{k}{2}$. One now takes an appropriate sum of the $\frac{k}{2}$ functions (6.61) in order to obtain a star-product projection on $\mathbb{T}^{2}$. By decoupling the theta-function in (6.61), it is possible to thereby show that the Weyl image of the resulting wavefunction coincides with the Boca projection in (6.39). A similar derivation of this noncommutative soliton on $\mathbb{T}^{2}$ is employed in [28], based on the construction of the noncommutative torus algebra $\mathcal{A}_{\theta}$ as the commutant of $\mathbb{Z}^{2}$ in the crossed product of the algebra $C^{\infty}\left(\mathbb{R}_{\Theta}^{2}\right)$ with the momentum lattice $\mathbb{Z}^{2}$.

\subsection{Matrix GMS Projections}

We will now apply the approximation of $\mathcal{A}_{\theta}$ described in section 3.2 to obtain a matrix regularization of the basic GMS soliton projections. We start from the $\theta \rightarrow 0$ limit of the Boca projection in (6.52), and apply the map (3.28) to get

$$
\Gamma_{n}\left(\boldsymbol{\psi}_{\theta}\right)=\theta \sum_{(m, r) \in \mathbb{Z}^{2}} \mathrm{e}^{-\frac{\pi \theta}{2}\left(m^{2}-2 \mathrm{i} m r+r^{2}\right)}\left(\mathrm{U}_{n}\right)^{m}\left(\mathrm{~V}_{n}\right)^{r}
$$

We will evaluate (6.62) by regarding it as a matrix-valued function on a pair of circles. Let us first examine the matrix elements corresponding to the first tower. By using (4.5), (4.6) and (4.10), we may write the $(i j)^{\text {th }}$ matrix 
element of (6.62) with $1 \leq i, j \leq q_{2 n}$ as

$$
\begin{aligned}
\left(\Gamma_{n}\left(\boldsymbol{\psi}_{\theta}\right)\right)_{i j}= & \theta \sum_{(k, l) \in \mathbb{Z}^{2}} z^{\kappa} \sum_{s=1}^{q_{2 n}}\left(\omega_{n}\right)^{s(i-1)} \exp \left[-\frac{\pi \theta}{2}\left(\left(s+l q_{2 n}\right)^{2}\right.\right. \\
& \left.\left.-2 \mathrm{i}\left(s+l q_{2 n}\right)\left(j-i+\kappa_{k} q_{2 n}\right)+\left(j-i+\kappa_{k} q_{2 n}\right)^{2}\right)\right],
\end{aligned}
$$

where the integer $\kappa_{k}$ depends on the circular Fourier momentum $k \in \mathbb{Z}$ and the triangular block of the matrix as

$$
\kappa_{k}:=\left\{\begin{array}{cc}
k & i<j \\
k+1 & i \geq j
\end{array}\right.
$$

We can transform the sums over $s \in \mathbb{Z}_{q_{2 n}}$ and $l \in \mathbb{Z}$ in (6.63) into a sum over a single integer $m=s+l q_{2 n} \in \mathbb{Z}$, and the sum over $k \in \mathbb{Z}$ to one over $r=\kappa_{k} \in \mathbb{Z}$. Unlike the continuum case, in the matrix regularization one cannot decouple the sums over $m$ and $r$, and instead of factorizing into the product of two genus one theta-functions as in (6.53), the matrix elements (6.63) can generically only be written in terms of genus two Jacobi thetafunctions

$$
\vartheta(\boldsymbol{\nu} \mid \mathrm{i} \boldsymbol{\sigma})=\sum_{\boldsymbol{m} \in \mathbb{Z}^{2}} \mathrm{e}^{-\pi \boldsymbol{m} \cdot \boldsymbol{\sigma} \boldsymbol{m}+2 \pi \mathrm{i} \boldsymbol{m} \cdot \boldsymbol{\nu}}
$$

where $\boldsymbol{m} \cdot \boldsymbol{\nu}:=m_{1} \nu_{1}+m_{2} \nu_{2}$. The functions (6.65) are holomorphic in $\boldsymbol{\nu} \in \mathbb{C}^{2}$ for symmetric $2 \times 2$ period matrices $\boldsymbol{\sigma}$ of positive definite real part.

With $z:=\mathrm{e}^{2 \pi \mathrm{i} \tau / r_{n}}, \tau \in\left[0, r_{n}\right)$, after some algebra we may thereby write (6.63) as

$$
\left(\Gamma_{n}\left(\boldsymbol{\psi}_{\theta}\right)\right)_{i j}=\theta \mathrm{e}^{-\frac{\pi \theta}{2}(i-j)^{2}} \vartheta\left(\boldsymbol{\nu}_{i j}^{(n)} \mid \mathrm{i} \boldsymbol{\sigma}^{(n)}\right)
$$

where

$$
\begin{aligned}
\boldsymbol{\nu}_{i j}^{(n)} & =\left(\begin{array}{c}
\mathrm{i}\left(\frac{p_{2 n}}{q_{2 n}}(i-1)+\frac{\theta}{2}(j-i)\right) \\
\frac{q_{2 n} \theta}{2}(i-j)+\frac{\mathrm{i} \tau}{r_{n}}
\end{array}\right), \\
\boldsymbol{\sigma}^{(n)} & =\frac{\theta}{2}\left(\begin{array}{cc}
1 & -\mathrm{i} q_{2 n} \\
-\mathrm{i} q_{2 n} & \left(q_{2 n}\right)^{2}
\end{array}\right) .
\end{aligned}
$$

The computation of the matrix elements corresponding to the second tower is completely analogous with the replacements $\left(p_{2 n}, q_{2 n}\right) \rightarrow\left(p_{2 n-1}, q_{2 n-1}\right)$, $\tau \rightarrow \tau^{\prime}$ and $r_{n} \rightarrow r_{n}^{\prime}$ in the above. In this way we arrive at the matrix approximation to the Boca projection $\mathrm{B}_{\theta}$ in the limit $\theta \rightarrow 0$ in the form

$$
\Gamma_{n}\left(\boldsymbol{\psi}_{\theta}\right)=\theta\left(\begin{array}{cc}
\left(\mathrm{e}^{-\frac{\pi \theta}{2}(i-j)^{2}} \vartheta\left(\boldsymbol{\nu}_{i j}^{(n)} \mid \mathrm{i} \boldsymbol{\sigma}^{(n)}\right)\right) & (0)_{q_{2 n} \times q_{2 n-1}} \\
(0)_{q_{2 n-1} \times q_{2 n}} & \left(\mathrm{e}^{-\frac{\pi \theta}{2}\left(i^{\prime}-j^{\prime}\right)^{2}} \vartheta\left(\boldsymbol{\nu}_{i^{\prime} j^{\prime}}^{\prime(n)} \mid \mathrm{i} \boldsymbol{\sigma}^{\prime(n)}\right)\right)
\end{array}\right),
$$


and thus the matrix regularization on the torus naturally involves hyperelliptic functions.

To transform to an appropriate small $\theta$ limit, as before we apply the Jacobi inversion formula for the genus two theta functions (6.65),

$$
\vartheta(\boldsymbol{\nu} \mid \mathrm{i} \boldsymbol{\sigma})=\frac{\mathrm{e}^{-\pi \boldsymbol{\nu} \cdot \boldsymbol{\sigma}^{-1} \boldsymbol{\nu}}}{\sqrt{\operatorname{det} \boldsymbol{\sigma}}} \vartheta\left(\text { i } \boldsymbol{\sigma}^{-1} \boldsymbol{\nu} \mid \text { i } \boldsymbol{\sigma}^{-1}\right)
$$

which now holds up to an irrelevant phase factor. By substituting (6.67) into (6.65) it is straightforward to show that the $\theta \rightarrow 0$ limit of the right-hand side of (6.69) also picks out the zero mode $\boldsymbol{m}=\mathbf{0}$. Then, from (6.66), (6.67) and (6.69), after some algebra we have in the first tower

$$
\begin{aligned}
\left(\Gamma_{n}\left(\boldsymbol{\psi}_{\theta}\right)\right)_{i j}= & \frac{1}{q_{2 n}} \mathrm{e}^{-\frac{\pi \theta}{2}(i-j)^{2}} \exp \left\{-\frac{\pi}{\left(q_{2 n}\right)^{2} \theta}\left[\left(p_{2 n}\right)^{2}(i-1)^{2}\right.\right. \\
& \left.\left.+2 \mathrm{i} p_{2 n}(i-1) \frac{\tau}{r_{n}}-\left(q_{2 n} \theta(i-j)+\frac{\mathrm{i} \tau}{r_{n}}\right)^{2}\right]\right\} .
\end{aligned}
$$

As before, we now substitute into (6.70) the rescaling (6.57) and the circular coordinates

$$
\frac{\tau}{r_{n}}=\frac{t}{2 \pi R}
$$

with $t \in \mathbb{R}$, and take the large area limit $R \rightarrow \infty$. Since $\theta \rightarrow 0$, it follows from (6.57) and appendix A, eq. (A.6) that $p_{2 n} \rightarrow 0$ as $p_{2 n} \sim q_{2 n} \theta \sim \frac{1}{R^{2}}$ also in this limit. A completely analogous analysis carries through for the second tower, and in this way we arrive finally at the matrix version of the basic GMS soliton (6.59) (or (6.58)) in the form

$$
\begin{aligned}
& \Gamma_{n}\left(\boldsymbol{\psi}_{\Theta}\right)\left(t, t^{\prime}\right) \\
& =\left(\begin{array}{cc}
\frac{1}{q_{2 n}} \mathrm{e}^{-t^{2} /\left(2 q_{2 n}\right)^{2} \Theta}(1)_{q_{2 n} \times q_{2 n}} & (0)_{q_{2 n} \times q_{2 n-1}} \\
(0)_{q_{2 n-1} \times q_{2 n}} & \frac{1}{q_{2 n-1}} \mathrm{e}^{-t^{\prime 2} /\left(2 q_{2 n-1}\right)^{2} \Theta(1)_{q_{2 n-1} \times q_{2 n-1}}}
\end{array}\right) .
\end{aligned}
$$

The matrix regularization (6.72) determines solitons on noncommutative $\mathbb{R}^{2}$ as approximate projections in the algebra $\mathbb{M}_{q_{2 n}}\left(C^{\infty}(\mathbb{R})\right) \oplus \mathbb{M}_{q_{2 n-1}}\left(C^{\infty}(\mathbb{R})\right)$ of matrix-valued functions on two copies of the real line $\mathbb{R}$. They are approximate in the sense that while the matrices $\frac{1}{q_{2 n}}(1)_{q_{2 n} \times q_{2 n}}, \frac{1}{q_{2 n-1}}(1)_{q_{2 n-1} \times q_{2 n-1}}$ are projection operators in $\mathbb{M}_{q_{2 n}}(\mathbb{C}), \mathbb{M}_{q_{2 n-1}}(\mathbb{C})$, the Gaussian prefactors in $S(\mathbb{R})$ combine to projections only in the $n \rightarrow \infty$ limit. After an appropriate rescaling of the coordinates $t, t^{\prime} \in \mathbb{R}$, the matrix soliton (6.72) evidently converges to the GMS one-soliton configuration (6.42), and in particular, by using translational symmetry, its moduli space is naturally isomorphic 
to $(\mathbb{R} \times \mathbb{N}) \times(\mathbb{R} \times \mathbb{N})$, where the extra factors of $\mathbb{N}$ come from the freedom in replacing the matrices $(1)_{q \times q}$ by $\frac{1}{k}(k)_{q \times q}$ and rescaling $q_{2 n} \rightarrow k q_{2 n}$, $q_{2 n-1} \rightarrow k q_{2 n-1}$ for any $k \in \mathbb{N}$ in the limit $n \rightarrow \infty$. Note, however, that since the two towers are independent, the soliton moduli space is determined by a pair of one-dimensional, localized matrix-valued functions on $\mathbb{R}$ and it is no longer a complex manifold at the finite level. Higher Landau levels can be approximated by constructing appropriate finite versions of the differential operators (6.43), similarly to section 4 , and applying them to the basic soliton fields (6.72), as in (6.44). The corresponding $k$-soliton moduli space will then be a symmetric orbifold of the single soliton one. We shall not pursue this construction any further here, but in any case this gives a precise way to regulate field theories on the noncommutative plane by means of matrix quantum mechanics.

\section{Acknowledgments}

We thank G. Elliott, D. Evans, L. Griguolo, J.-H. Park and J. Varilly for helpful discussions and correspondence. The work of G.L. and F.L. is supported in part by the Progetto di Ricerca di Interesse Nazionale SINTESI. The work of R.J.S. is supported in part by an Advanced Fellowship from the Particle Physics and Astronomy Research Council (U.K.). F.L. would like to thank the Department of Mathematics at Heriot-Watt University for the hospitality presented to him during several extended visits in his sabbatical year.

\section{A Continued Fraction Expansions}

A well known result of number theory [36] states that any irrational number $\theta$ can be uniquely represented as a simple continued fraction expansion

$$
\theta=\lim _{n \rightarrow \infty} \theta_{n}, \quad \theta_{n}:=\frac{p_{n}}{q_{n}}
$$

involving positive integers $c_{k}>0, k \geq 1$ and $c_{0} \in \mathbb{Z}$. The continued fraction is the definition of a sequence of rational numbers $\left\{\theta_{n}\right\}$ (the approximants), which converge to $\theta$. The $n^{\text {th }}$ approximant $\theta_{n}$ of the expansion is given by

$$
\theta_{n}=c_{0}+\frac{1}{c_{1}+\frac{1}{c_{2}+\frac{1}{\ddots c_{n-1}+\frac{1}{c_{n}}}}} .
$$


A short-hand notation for the expansion is

$$
\theta=\left[c_{0}, c_{1}, c_{2}, \ldots\right] .
$$

The relatively prime integers $p_{n}$ and $q_{n}$ in (A.1) may be computed recursively from (A.2) by using the formulæ

$$
\begin{aligned}
& p_{n}=c_{n} p_{n-1}+p_{n-2} \quad, \quad p_{0}=c_{0}, p_{1}=c_{0} c_{1}+1, \\
& q_{n}=c_{n} q_{n-1}+q_{n-2} \quad, \quad q_{0}=1, q_{1}=c_{1}
\end{aligned}
$$

for $n \geq 2$. Note that all $q_{n}>0$ while $p_{n} \in \mathbb{Z}$, and that both $q_{n}$ and $\left|p_{n}\right|$ are strictly increasing sequences which therefore diverge as $n \rightarrow \infty$. The sequence of convergents (A.2) can be shown to satisfy the bound

$$
\left|\theta-\theta_{n}\right| \leq \frac{1}{\left(q_{n}\right)^{2}}
$$

showing how fast the limit in (A.1) converges.

When $0<\theta<1$ (so that $p_{0}=c_{0}=0$ and all $p_{n}>0$ ), the even order convergents are always smaller than $\theta$, while the odd order ones are larger. Thus the even (resp. odd) order convergents form an increasing (resp. decreasing) sequence which converges to $\theta$ as

$$
\theta_{2 n-2}<\theta_{2 n}<\theta<\theta_{2 n+1}<\theta_{2 n-1} .
$$

Furthermore, the approximants fulfill Diophantine properties

$$
\left(\begin{array}{ll}
p_{2 n \pm 1} & p_{2 n} \\
q_{2 n \pm 1} & q_{2 n}
\end{array}\right) \in S L(2, \mathbb{Z})
$$

which follow from the recursion relations (A.4) by induction on $n$. We also define the decreasing sequence

$$
\begin{aligned}
\beta_{2 n} & =p_{2 n-1}-q_{2 n-1} \theta=q_{2 n-1}\left(\theta_{2 n-1}-\theta\right), \\
\beta_{2 n-1} & =q_{2 n} \theta-p_{2 n}=q_{2 n}\left(\theta-\theta_{2 n}\right),
\end{aligned}
$$

with $\beta_{k}>0$ and $\lim _{k \rightarrow \infty} \beta_{k}=0$. For each $n$ the properties (A.7) imply that

$$
q_{2 n} \beta_{2 n}+q_{2 n-1} \beta_{2 n-1}=1, \quad q_{2 n} \beta_{2 n+2}+q_{2 n+1} \beta_{2 n-1}=1 .
$$

\section{B Matrix Unit Relations}

To prove that the collections of operators $\left\{\mathrm{P}^{i i}\right\}$ and $\left\{\mathrm{P}^{i+2, i+1}\right\}$ in $(3.4)$ and (3.9) satisfy the relations

$$
\mathrm{P}^{i j} \mathrm{P}^{k l}=\delta^{j k} \mathrm{P}^{i l}
$$


we will first prove that

$$
\mathrm{P}^{21} \mathrm{P}^{i i}=0 \quad \forall i>1
$$

For this, we will show that $\mathrm{P}^{21} \mathrm{P}^{i i}|\psi\rangle=0$ for all vectors $|\psi\rangle \in \mathcal{H}$ of the underlying Hilbert space on which the algebra $\mathcal{A}_{\theta}$ is represented. If $|\psi\rangle$ has no component in the subspace $\mathcal{H}_{i}=\operatorname{im}\left(\mathrm{P}^{i i}\right)$, then this is trivially true, so we suppose that $|\psi\rangle \in \mathcal{H}_{i}$. Note that the operator $\Pi^{21}$ in (3.5) contains the projection $\mathrm{P}^{11}$ to its extreme right. With this observation, we can now exploit a standard result of functional analysis (see for instance [61, Theorem 2.3.4]) which states that the kernel of a bounded linear operator coincides with the kernel of the partial isometry in its polar decomposition. Since $i>1$, we have $\mathrm{P}^{11}|\psi\rangle=0$, and so

$$
\mathcal{H}_{i} \subset \operatorname{ker}\left(\Pi^{21}\right)=\operatorname{ker}\left(\mathrm{P}^{21}\right)
$$

It follows that for any vector $|\psi\rangle \in \mathcal{H}$ we have

$$
\mathrm{P}^{i i}|\psi\rangle \in \operatorname{ker}\left(\mathrm{P}^{21}\right)
$$

for $i>1$, which establishes (B.2). By repeating this argument for the adjoint operator $\left(\Pi^{21}\right)^{\dagger}$ and using the definition (3.10) one similarly proves

$$
\mathrm{P}^{12} \mathrm{P}^{11}=0
$$

We will now prove the identity

$$
\mathrm{P}^{21} \mathrm{P}^{11}=\mathrm{P}^{21}
$$

For this, we decompose a generic vector $|\psi\rangle \in \mathcal{H}$ as

$$
|\psi\rangle=\left|\psi_{1}\right\rangle \oplus\left|\psi_{1}^{\perp}\right\rangle
$$

with $\left|\psi_{1}\right\rangle \in \mathcal{H}_{1}$ and $\left|\psi_{1}^{\perp}\right\rangle \in \mathcal{H}_{1}^{\perp}$. Then

$$
\mathrm{P}^{21} \mathrm{P}^{11}|\psi\rangle=\mathrm{P}^{21}\left|\psi_{1}\right\rangle
$$

and the desired result now follows from the fact that

$$
\left|\psi_{1}^{\perp}\right\rangle \in \operatorname{ker}\left(\mathrm{P}^{11}\right) \subset \operatorname{ker}\left(\Pi^{21}\right)=\operatorname{ker}\left(\mathrm{P}^{21}\right) .
$$

Finally, to establish the expression

$$
\mathrm{P}^{22} \mathrm{P}^{21}=\mathrm{P}^{21}
$$


we note first of all that since the operator $\Pi^{21}$ contains an orthogonal projection on its right, it has closed range ${ }^{8}$. With this observation, we can now exploit another standard functional analytic result (see for instance [77, Theorem 15.3.8]) which states that the range of a closed operator is the same as the range of the partial isometry in its polar decomposition. It follows from this, and from the fact that the operator $\Pi^{21}$ contains the projection $\mathrm{P}^{22}$ to its extreme left, that

$$
\mathrm{P}^{21}(\mathcal{H})=\Pi^{21}(\mathcal{H}) \subset \mathcal{H}_{2}
$$

Since $\mathrm{P}^{22}$ acts as the identity operator on the subspace $\mathcal{H}_{2}$, the relation (B.10) follows. The corresponding identities for the projections and partial isometries with index labels larger than 2 can then be constructed either by direct multiplication or by use of the automorphism $\alpha$ defined in (3.2).

\section{Approximating the Torus Algebra}

The proof of the fact that

$$
\lim _{n \rightarrow \infty}\left\|a-\Gamma_{n}(a)\right\|_{0}=0
$$

with $a \in \mathcal{A}_{\theta}$ and $\Gamma_{n}$ the projection onto the finite level subalgebra $\mathcal{A}_{n}$ in (3.18), comes from repeated applications of the triangle and product inequalities for the $C^{*}$-norm. For any $m, r \in \mathbb{Z}$, by using the fact that $\left\|\left(\mathrm{U}_{n}\right)^{m}\right\|_{0}=\left\|\left(\mathrm{V}_{n}\right)^{r}\right\|_{0}=1$ and $\left\|U^{m}\right\|_{0}=\left\|V^{r}\right\|_{0}=1$, we have

$$
\left\|\left(\mathrm{U}_{n}\right)^{m}\left(\mathrm{~V}_{n}\right)^{r}-U^{m} V^{r}\right\|_{0} \leq\left\|\left(\mathrm{U}_{n}\right)^{m}-U^{m}\right\|_{0}+\left\|\left(\mathrm{V}_{n}\right)^{r}-V^{r}\right\|_{0} .
$$

Using (3.26), we now define $\mathrm{U}_{n}=U+\Delta_{n}$ with $\left[U, \Delta_{n}\right]=0$ and $\left\|\Delta_{n}\right\|_{0} \leq \varepsilon_{n}$. Then

$$
\begin{aligned}
\left\|\left(\mathrm{U}_{n}\right)^{m}-U^{m}\right\|_{0} & =\left\|\sum_{p=1}^{m}\left(\begin{array}{c}
m \\
p
\end{array}\right) U^{m-p}\left(\Delta_{n}\right)^{p}\right\|_{0} \\
& \leq \sum_{p=1}^{m}\left(\begin{array}{c}
m \\
p
\end{array}\right)\left(\left\|\Delta_{n}\right\|_{0}\right)^{p} \\
& \leq 1-\left(1-\varepsilon_{n}\right)^{m}<m \varepsilon_{n} .
\end{aligned}
$$

\footnotetext{
${ }^{8}$ The statement that a bounded linear operator $T$ is closed is equivalent to the following statements [77]: (a) 0 is an isolated point in the spectrum of the self-adjoint operators $T^{\dagger} T$ and $T T^{\dagger}$; (b) the right and left ideals $T \mathcal{A}_{\theta}$ and $\mathcal{A}_{\theta} T$ are closed in the norm topology on $\mathcal{A}_{\theta}$; and (c) there exist projection operators $\mathrm{P}$ and $\mathrm{Q}$ with $\mathcal{A}_{\theta} T=\mathcal{A}_{\theta} \mathrm{P}$ and $T \mathcal{A}_{\theta}=\mathrm{Q} \mathcal{A}_{\theta}$.
} 
A completely analogous calculation gives

$$
\left\|\left(\mathrm{V}_{n}\right)^{r}-V^{r}\right\|_{0}<r \varepsilon_{n}
$$

From (3.28) we have

$$
\left\|a-\Gamma_{n}(a)\right\|_{0} \leq \sum_{(m, r) \in \mathbb{Z}^{2}}\left|a_{m, r}\right|\left\|\left(\mathrm{U}_{n}\right)^{m}\left(\mathrm{~V}_{n}\right)^{r}-U^{m} V^{r}\right\|_{0}
$$

and so from (C.2)-(C.4) it follows that

$$
\left\|a-\Gamma_{n}(a)\right\|_{0}<\varepsilon_{n} \sum_{(m, r) \in \mathbb{Z}^{2}}(m+r)\left|a_{m, r}\right|
$$

which vanishes as $n \rightarrow \infty$ for Schwartz sequences $\left\{a_{m, r}\right\}$. Therefore, to each element of $\mathcal{A}_{\theta}$ there always corresponds an element of the subalgebra $\mathcal{A}_{n}$ to within an arbitrarily small radius in norm.

\section{Inductive Limit}

In this appendix we will show how to obtain the noncommutative torus as an inductive limit ${ }^{9}$

$$
\mathcal{A}_{\theta}=\bigcup_{n=0}^{\infty} \mathcal{B}_{n}
$$

of an appropriate inductive system of algebras $\left\{\mathcal{B}_{n}, \iota_{n}\right\}_{n \geq 0}$, together with injective unital $*$-morphisms $\iota_{n}: \mathcal{B}_{n} \hookrightarrow \mathcal{B}_{n+1}[26]$. It turns out that for K-theoretical reasons one needs to take $\mathcal{B}_{n}=\mathcal{A}_{2 n+1}=\mathbb{M}_{q 4 n+2}\left(C^{\infty}\left(\mathbb{S}^{1}\right)\right) \oplus$ $\mathbb{M}_{q_{4 n+1}}\left(C^{\infty}\left(\mathbb{S}^{1}\right)\right)$. The crucial point is to explicitly construct the embeddings $\iota_{n}$ in such a way that the K-theory groups (2.34) and (2.36) of the noncommutative torus are obtained in the limit out of the "finite level" counterparts (3.33). For this, one needs to exploit the continued fraction expansion of $\theta$ and the recursion relations (A.4) in a very careful manner.

It is well known [25] that on the "matrix part", the continued fraction expansion of $\theta$ directly gives the required dimension group of the torus $\mathcal{A}_{\theta}$ while leading to a trivial $\mathrm{K}_{1}$-group, this being the appropriate setting for immersions into an AF-algebra [65]. In the present case, in order to get the correct $\mathrm{K}_{1}$-group (2.36), which is generated by the two independent classes

\footnotetext{
${ }^{9}$ Strictly speaking, the following discussion is only rigorously valid in the continuous category. However, on the noncommutative torus the proof of [26] should go through also for smooth functions, with the appropriate technical modifications.
} 
$[U]$ and $[V]$, one needs to modify the construction somewhat. The main point is that the clock operators appearing in (3.20) are elements of finitedimensional matrix algebras and therefore have trivial $\mathrm{K}_{1}$-class. The nontrivial group is thereby generated by the generalized shift operators in (3.20), and this must be kept in mind when embedding from one level to the next. To this end, one "skips" a step [26], by going from level $n$ to level $n+1$ (so as to send $q_{n}$ to $\left.q_{n+4}\right)$. With the skipping of steps, the roles of the clock and shift operators in (3.20) change from one level to the next.

We thereby use the recursion relations (A.4) to define integer valued matrices

$$
P_{n}=\left(\begin{array}{ll}
r_{n} & s_{n} \\
t_{n} & u_{n}
\end{array}\right), n=0,1,2, \ldots
$$

with the property that

$$
P_{n}\left(\begin{array}{c}
q_{4 n+2} \\
q_{4 n+1}
\end{array}\right)=\left(\begin{array}{c}
q_{4(n+1)+2} \\
q_{4(n+1)+1}
\end{array}\right)
$$

To simplify notation for the rest of this appendix, let us denote $d_{n}=q_{4 n+2}$ and $d_{n}^{\prime}=q_{4 n+1}$. As mentioned before, we shall take the algebra in the inductive limit to be

$$
\mathcal{B}_{n}=\mathcal{A}_{2 n+1}=\mathbb{M}_{d_{n}}\left(C^{\infty}\left(\mathbb{S}^{1}\right)\right) \oplus \mathbb{M}_{d_{n}^{\prime}}\left(C^{\infty}\left(\mathbb{S}^{1}\right)\right), \quad n=0,1,2, \ldots
$$

and we are now ready to describe the embedding $\iota_{n}: \mathcal{B}_{n} \hookrightarrow \mathcal{B}_{n+1}$.

Since a generic element of the matrix algebra (D.4) is of the form

$$
\mathrm{a}_{n}=\sum_{k \in \mathbb{Z}} \sum_{i, j=1}^{d_{n}} a_{i j ; k}^{(n)} z^{k} \mathrm{P}_{n}^{i j} \oplus \sum_{k^{\prime} \in \mathbb{Z}} \sum_{i^{\prime}, j^{\prime}=1}^{d_{n}^{\prime}} a_{i^{\prime} j^{\prime} ; k^{\prime}}^{(n)} z^{\prime k^{\prime}} \mathrm{P}_{n}^{\prime i^{\prime} j^{\prime}}
$$

it suffices to give the immersions of the $d_{n} \times d_{n}$ and $d_{n}^{\prime} \times d_{n}^{\prime}$ matrices $\left(a_{i j ; k}^{(n)}\right)$ and $\left(a_{i^{\prime} j^{\prime} ; k^{\prime}}^{\prime(n)}\right)$ for given fixed values of the circular Fourier modes $k$ and $k^{\prime}$, and of the two unitaries $z$ and $z^{\prime}$ which generate the center of the algebra $\mathcal{A}_{n}$. Denoting the mode restrictions by $\mathrm{a}_{n} \uparrow_{k}$, the embedding of the matrix 
degrees of freedom is given by

$$
\begin{aligned}
& \iota_{n}\left[\left(\begin{array}{ll}
\mathrm{a}_{n} \uparrow_{k} & \\
& (0)_{d_{n}^{\prime} \times d_{n}^{\prime}}
\end{array}\right)\right] \\
& =\left(\begin{array}{cccc}
\mathbb{1}_{r_{n}} \otimes\left(a_{i j ; k}^{(n)}\right) & & & \\
& (0)_{s_{n} d_{n}^{\prime} \times s_{n} d_{n}^{\prime}} & & \\
& & \mathbb{1}_{t_{n}} \otimes\left(a_{i j ; k}^{(n)}\right) & \\
& & & (0)_{u_{n} d_{n}^{\prime} \times u_{n} d_{n}^{\prime}}
\end{array}\right), \\
& \iota_{n}\left[\left(\begin{array}{cc}
(0)_{d_{n} \times d_{n}} & \\
& \left.\mathrm{a}_{n}\right\rceil_{k^{\prime}}
\end{array}\right)\right] \\
& =\left(\begin{array}{cccc}
(0)_{r_{n} d_{n} \times r_{n} d_{n}} & & & \\
& \mathbb{1}_{s_{n}} \otimes\left(a_{i^{\prime} j^{\prime} ; k^{\prime}}^{\prime(n)}\right) & & \\
& & (0)_{t_{n} d_{n} \times t_{n} d_{n}} & \\
& & & \mathbb{1}_{u_{n}} \otimes\left(a_{i^{\prime} j^{\prime} ; k^{\prime}}^{\prime(n)}\right.
\end{array}\right),
\end{aligned}
$$

while $z$ and $z^{\prime}$ are embedded as

$$
\begin{aligned}
& \iota_{n}\left[\left(\begin{array}{ll}
z \mathbb{1}_{d_{n}} & \\
& (0)_{d_{n}^{\prime} \times d_{n}^{\prime}}
\end{array}\right)\right] \\
& =\left(\begin{array}{cccc}
\mathcal{S}_{r_{n}}(z) \otimes \mathbb{1}_{d_{n}} & & & \\
& (0)_{s_{n} d_{n}^{\prime} \times s_{n} d_{n}^{\prime}} & & \\
& & \mathcal{S}_{t_{n}}(1) \otimes \mathbb{1}_{d_{n}} & \\
& & (0)_{u_{n} d_{n}^{\prime} \times u_{n} d_{n}^{\prime}}
\end{array}\right), \\
& \iota_{n}\left[\left(\begin{array}{cc}
(0)_{d_{n} \times d_{n}} & \\
& z^{\prime} \mathbb{1}_{d_{n}^{\prime}}
\end{array}\right)\right] \\
& =\left(\begin{array}{cccc}
(0)_{r_{n} d_{n} \times r_{n} d_{n}} & & & \\
& \mathcal{S}_{s_{n}}(1) \otimes \mathbb{1}_{d_{n}^{\prime}} & & \\
& & (0)_{t_{n} d_{n} \times t_{n} d_{n}} & \\
& & & \mathcal{S}_{u_{n}}\left(z^{\prime}\right) \otimes \mathbb{1}_{d_{n}^{\prime}}
\end{array}\right) .
\end{aligned}
$$

When lifted to the K-theory groups, the homomorphism $\iota_{n}$ acts as the matrix $P_{n}$ on $\mathrm{K}_{0}\left(\mathcal{B}_{n}\right)=\mathbb{Z} \oplus \mathbb{Z}$ and as the identity on $\mathrm{K}_{1}\left(\mathcal{B}_{n}\right)=\mathbb{Z} \oplus \mathbb{Z}$, so that the inductive limit algebra has the appropriate K-theory groups (2.34)-(2.36). Furthermore, in [26] it is shown that the limit algebra is a simple unital algebra that has a unique trace state. All of these properties select the noncommutative torus algebra $\mathcal{A}_{\theta}$ up to isomorphism. 


\section{E Approximating the Leibniz Rule}

In this appendix we will show that the two "derivatives" defined in (4.8) satisfy an approximate Leibniz rule, which becomes the usual one in the $n \rightarrow$ $\infty$ limit. To keep formulæ from becoming overly cumbersome, we will only indicate explicitly terms appearing in the first tower. Analogous expressions are always understood to appear in the second tower. We will denote by $[a]_{q}$ the integer part of a real number $a$ modulo $q$, with the convention that $[a]_{0}$ is its integer part in $\mathbb{Z}$.

The product of two elements of the finite level algebra $\mathcal{A}_{n}$ with expansion (4.5) is given by

$$
\begin{aligned}
\mathrm{a}_{n} \mathrm{~b}_{n}= & \sum_{i, j, s, t=1}^{q_{2 n}} \sum_{k, l \in \mathbb{Z}} \alpha_{i+\left[\frac{q_{2 n}}{2}\right]_{0}, j ; k}^{(n)} \beta_{s+\left[\frac{q_{2 n}}{2}\right]_{0}, t ; l}^{(n)}\left(\omega_{n}\right)^{j s-i t} z^{k+l+\left[\frac{j+t}{q_{2 n}}\right]_{0}} \\
& \times\left(\mathcal{C}_{q_{2 n}}\right)^{[i+s]_{q_{2 n}}}\left(\mathcal{S}_{q_{2 n}}(z)\right)^{[j+t]_{q_{2 n}}} \\
& \oplus \text { (second tower) } .
\end{aligned}
$$

By using (4.8) one may calculate the derivative of the product (E.1) to be

$$
\begin{aligned}
\nabla_{1}\left(\mathrm{a}_{n} \mathrm{~b}_{n}\right)= & 2 \pi \mathrm{i} \sum_{i, j, s, t=1}^{q_{2 n}} \sum_{k, l \in \mathbb{Z}}[i+s]_{q_{2 n}} \alpha_{i+\left[\frac{q_{2 n}}{2}\right]_{0}, j ; k}^{(n)} \beta_{s+\left[\frac{q_{2 n}}{2}\right]_{0}, t ; l}^{(n)} \\
& \times\left(\omega_{n}\right)^{j s-i t} z^{k+l+\left[\frac{j+t}{q_{2 n}}\right]_{0}}\left(\mathcal{C}_{q_{2 n}}\right)^{[i+s]_{q_{2 n}}}\left(\mathcal{S}_{q_{2 n}}(z)\right)^{[j+t]_{q_{2 n}}} \\
& \oplus \text { (second tower) }
\end{aligned}
$$

while a direct calculation using the definition (4.8) and the product formula (E.1) gives

$$
\begin{aligned}
\left(\nabla_{1} \mathrm{a}_{n}\right) \mathrm{b}_{n}+\mathrm{a}_{n}\left(\boldsymbol{\nabla}_{1} \mathrm{~b}_{n}\right)= & 2 \pi \mathrm{i} \sum_{i, j, s, t=1}^{q_{2 n}} \sum_{k, l \in \mathbb{Z}}(i+s) \\
& \times \alpha_{i+\left[\frac{q_{2 n}}{2}\right]_{0}, j ; k}^{(n)} \beta_{s+\left[\frac{q_{2 n}}{2}\right]_{0}, t ; l}^{(n)} \\
& \times\left(\omega_{n}\right)^{j s-i t} z^{k+l+\left[\frac{j+t}{q_{2 n}}\right]_{0}} \\
& \times\left(\mathcal{C}_{q_{2 n}}\right)^{[i+s]_{q_{2 n}}}\left(\mathcal{S}_{q_{2 n}}(z)\right)^{[j+t]_{q_{2 n}}} \\
& \oplus(\text { second tower }) .
\end{aligned}
$$

The difference between these two expressions occurs when at least one of the integers $i$ or $s$ is of order $\left[\frac{q_{2 n}}{2}\right]_{0}$, in which case the corresponding coefficient 
of $\mathrm{a}_{n}$ or $\mathrm{b}_{n}$ is exponentially small in the limit. The two expressions thereby coincide at $n \rightarrow \infty$. A completely analogous computation gives

$$
\begin{aligned}
\nabla_{2}\left(\mathrm{a}_{n} \mathrm{~b}_{n}\right)= & 2 \pi \mathrm{i} \sum_{i, j, s, t=1}^{q_{2 n}} \sum_{k, l \in \mathbb{Z}}\left\{[j+t]_{q_{2 n}}+q_{2 n}\left(k+l+\left[\frac{j+t}{q_{2 n}}\right]_{0}\right)\right\} \\
& \times \alpha_{i+\left[\frac{q_{2 n}}{2}\right]_{0}, j ; k}^{(n)} \beta_{s+\left[\frac{q_{2 n}}{2}\right]_{0}, t ; l}^{(n)}\left(\omega_{n}\right)^{j s-i t} z^{k+l+\left[\frac{j+t}{q_{2 n}}\right]_{0}} \\
& \times\left(\mathcal{C}_{q_{2 n}}\right)^{[i+s]_{2 n}}\left(\mathcal{S}_{q_{2 n}}(z)\right)^{[j+t]_{q_{2 n}}} \\
& \oplus \text { (second tower) }
\end{aligned}
$$

and

$$
\begin{aligned}
\left(\nabla_{2} \mathrm{a}_{n}\right) \mathrm{b}_{n}+\mathrm{a}_{n}\left(\boldsymbol{\nabla}_{2} \mathrm{~b}_{n}\right)= & 2 \pi \mathrm{i} \sum_{i, j, s, t=1}^{q_{2 n}} \sum_{k, l \in \mathbb{Z}}\left(j+t+q_{2 n}(k+l)\right) \\
& \times \alpha_{i+\left[\frac{q_{2 n}}{2}\right]_{0}, j ; k}^{(n)} \beta_{s+\left[\frac{q_{2 n}}{2}\right]_{0}, t ; l}^{(n)} \\
& \times\left(\omega_{n}\right)^{j s-i t} z^{k+l+\left[\frac{j+t}{q_{2 n}}\right]_{0}} \\
& \times\left(\mathcal{C}_{q_{2 n}}\right)^{[i+s]_{q_{2 n}}}\left(\mathcal{S}_{q_{2 n}}(z)\right)^{[j+t]_{q_{2 n}}} \\
& \oplus(\text { second tower }) .
\end{aligned}
$$

Again the two expressions differ only for large momenta $j$ and $t$.

\section{References}

[1] M. Aganagic, R. Gopakumar, S. Minwalla and A. Strominger, Unstable Solitons in Noncommutative Gauge Theory, J. High Energy Phys. 0104 (2001) 001 [hep-th/0009142].

[2] S.Yu. Alexandrov, V.A. Kazakov and D. Kutasov, Nonperturbative Effects in Matrix Models and D-Branes, J. High Energy Phys. 0309 (2003) 057 [hep-th/0306177].

[3] J. Ambjørn and S. Catterall, Stripes from (Noncommutative) Stars, Phys. Lett. B549 (2002) 253 [hep-lat/0209106].

[4] J. Ambjørn, Y.M. Makeenko, J. Nishimura and R.J. Szabo, Finite N Matrix Models of Noncommutative Gauge Theory, J. High Energy Phys. 9911 (1999) 029 [hep-th/9911041].

[5] J. Ambjørn, Y.M. Makeenko, J. Nishimura and R.J. Szabo, Lattice Gauge Fields and Discrete Noncommutative Yang-Mills Theory, J. High Energy Phys. 0005 (2000) 023 [hep-th/0004147]. 
[6] H. Aoki, N. Ishibashi, S. Iso, H. Kawai, Y. Kitazawa and T. Tada, Noncommutative Yang-Mills in IIB Matrix Model, Nucl. Phys. B565 (2000) 176 [hep-th/9908141].

[7] A.P. Balachandran, K. Gupta and S. Kürkçüoğlu, Edge Currents in Noncommutative Chern-Simons Theory from a New Matrix Model, J. High Energy Phys. 0309 (2003) 007 [hep-th/0306255].

[8] T. Banks, W. Fischler, S.H. Shenker and L. Susskind, M-Theory as a Matrix Model: A Conjecture, Phys. Rev. D55 (1997) 5112 [hep-th/9610043].

[9] I. Bars, H. Kajiura, Y. Matsuo and T. Takayanagi, Tachyon Condensation on Noncommutative Torus, Phys. Rev. D63 (2001) 086001 [hep-th/0010101].

[10] A.A. Belavin and A.M. Polyakov, Metastable States of TwoDimensional Isotropic Ferromagnets, JETP Lett. 22 (1975) 245.

[11] C. Becchi, S. Giusto and C. Imbimbo, The Wilson-Polchinski Renormalization Group Equation in the Planar Limit, Nucl. Phys. B633 (2002) 250 [hep-th/0202155].

[12] D. Berenstein, J. Maldacena and H. Natase, Strings in Flat Space and $p p$-Waves from $\mathcal{N}=4$ Super Yang-Mills, J. High Energy Phys. 0204 (2002) 013 [hep-th/0202021].

[13] W. Bietenholz, F. Hofheinz and J. Nishimura, Noncommutative Field Theories beyond Perturbation Theory, Fortsch. Phys. 51 (2003) 745 [hep-th/0212258].

[14] F.-P. Boca, Projections in Rotation Algebras and Theta Functions, Commun. Math. Phys. 202 (1999) 325 [math.0A/9803134].

[15] F.-P. Boca, Rotation $C^{*}$-Algebras and Almost Mathieu Operators (The Theta Foundation, 2001).

[16] A. Connes, $C^{*}$-Algèbres et Géométrie Différentielle, C.R. Acad. Sci. Paris $A 290$ (1980) 599; translated in: A. Connes, $C^{*}$-Algebras and Differential Geometry, hep-th/0101093.

[17] A. Connes, Noncommutative Geometry (Academic Press, 1994).

[18] A. Connes and M.A. Rieffel, Yang-Mills for Noncommutative Two-Tori, in: Operator Algebras and Mathematical Physics, Contemp. Math. 62 (1987) 237.

[19] A. Connes, M.R. Douglas and A. Schwarz, Noncommutative Geometry and Matrix Theory: Compactification on Tori, J. High Energy Phys. 9802 (1998) 003 [hep-th/9711162]. 
[20] L. Dạbrowski, T. Krajewski and G. Landi, Some Properties of NonLinear $\sigma$-Models in Noncommutative Geometry, Int. J. Mod. Phys. B14 (2000) 2367 [hep-th/0003099].

[21] L. Dąbrowski, T. Krajewski and G. Landi, Non-Linear $\sigma$-Models in Noncommutative Geometry: Fields with Values in Finite Spaces, Mod. Phys. Lett. A18 (2003) 2371 [math.QA/0309143].

[22] L. Dąbrowski, T. Krajewski and G. Landi, unpublished.

[23] K. Dasgupta, S. Mukhi and G. Rajesh, Noncommutative Tachyons, J. High Energy Phys. 0006 (2000) 022 [hep-th/0005006].

[24] M.R. Douglas and N.A. Nekrasov, Noncommutative Field Theory, Rev. Mod. Phys. 73 (2002) 977 [hep-th/0106048].

[25] E.G. Effros and C.L. Shen, Approximately Finite $C^{*}$-Algebras and Continued Fractions, Indiana Univ. Math. J. 29 (1980) 191.

[26] G.A. Elliott and D.E. Evans, The Structure of the Irrational Rotation $C^{*}$-Algebra, Ann. Math. 138 (1993) 477.

[27] G.A. Elliott and Q. Lin, Cut-Down Methods in the Inductive Limit Decomposition of Noncommutative Tori, J. London Math. Soc. 54 (1996) 121.

[28] R. Gopakumar, M. Headrick and M. Spradlin, On Noncommutative Multi-Solitons, Commun. Math. Phys. 233 (2003) 355 [hep-th/0103256].

[29] R. Gopakumar, S. Minwalla and A. Strominger, Noncommutative Solitons, J. High Energy Phys. 0005 (2000) 020 [hep-th/0003160].

[30] J.M. Gracia-Bondia and J.C. Varilly, Algebras of Distributions suitable for Phase Space Quantum Mechanics 1, J. Math. Phys. 29 (1988) 869.

[31] J.M. Gracia-Bondia, J.C. Varilly and H. Figueroa, Elements Of Noncommutative Geometry (Birkhauser, 2000).

[32] L. Griguolo and D. Seminara, Classical Solutions of the TEK Model and Noncommutative Instantons in Two Dimensions, hep-th/0311041.

[33] H. Grosse and R. Wulkenhaar, Renormalization of $\phi^{4}$ Theory on Noncommutative $\mathbb{R}^{2}$ in the Matrix Base, J. High Energy Phys. 0312 (2003) 019 [hep-th/0307017].

[34] S.S. Gubser and S.L. Sondhi, Phase Structure of Noncommutative Scalar Field Theories, Nucl. Phys. B605 (2001) 395 [hep-th/0006119].

[35] Z. Guralnik, R.C. Helling, K. Landsteiner and E. Lopez, Perturbative Instabilities on the Noncommutative Torus, Morita Duality and Twisted Boundary Conditions, J. High. Energy Phys. 0205 (2002) 025 [hep-th/0204037]. 
[36] G.H. Hardy and E.M. Wright, An Introduction to the Theory of Numbers (Oxford, 1954).

[37] J.A. Harvey, P. Kraus and F. Larsen, Exact Noncommutative Solitons, J. High Energy Phys. 0012 (2000) 024 [hep-th/0010060].

[38] J.A. Harvey, P. Kraus, F. Larsen and E.J. Martinec, D-Branes and Strings as Noncommutative Solitons, J. High Energy Phys. 0007 (2000) 042 [hep-th/0005031].

[39] B.-Y. Hou, D.-T. Peng, K.-J. Shi and R.-H. Yue, Solitons on Noncommutative Torus as Elliptic Calogero-Gaudin Models, Branes and Laughlin Wavefunctions, Int. J. Mod. Phys. A18 (2003) 2477 [hep-th/0204163].

[40] H. Kajiura, Y. Matsuo and T. Takayanagi, Exact Tachyon Condensation on Noncommutative Torus, J. High Energy Phys. 0106 (2001) 041 [hep-th/0104143].

[41] I.R. Klebanov, J. Maldacena and N. Seiberg, D-Brane Decay in TwoDimensional String Theory, J. High Energy Phys. 0307 (2003) 045 [hep-th/0305159].

[42] A. Konechny and A. Schwarz, An Introduction to Matrix Theory and Noncommutative Geometry, Phys. Rept. 360 (2002) 353 [hep-th/0012145, 0107251].

[43] T. Krajewski and M. Schnabl, Exact Solitons on Noncommutative Tori, J. High Energy Phys. 0108 (2001) 002 [hep-th/0104090].

[44] G. Landi, An Introduction to Noncommutative Spaces and their Geometries (Springer, 1997) [hep-th/9701078].

[45] G. Landi, F. Lizzi and R.J. Szabo, String Geometry and the Noncommutative Torus, Commun. Math. Phys. 206 (1999) 603 [hep-th/9806099].

[46] G. Landi, F. Lizzi and R.J. Szabo, From Large $N$ Matrices to the Noncommutative Torus, Commun. Math. Phys. 217 (2001) 181 [hep-th/9912130].

[47] G. Landi, F. Lizzi and R.J. Szabo, A New Matrix Model for Noncommutative Field Theory, Phys. Lett. B578 (2004) 449 [hep-th/0309031].

[48] E. Langmann and R.J. Szabo, Duality in Scalar Field Theory on Noncommutative Phase Spaces, Phys. Lett. B533 (2002) 168 [hep-th/0202039].

[49] E. Langmann, R.J. Szabo and K. Zarembo, Exact Solution of Quantum Field Theory on Noncommutative Phase Spaces, J. High Energy Phys. 0401 (2004) 017 [hep-th/0308043]. 
[50] U. Lindström, M. Rocek and R. von Unge, Noncommutative Soliton Scattering, J. High Energy Phys. 0012 (2000) 004 [hep-th/0008108].

[51] F. Lizzi, R.J. Szabo and A. Zampini, Geometry of the Gauge Algebra in Noncommutative Yang-Mills Theory, J. High Energy Phys. 0108 (2001) 032 [hep-th/0107115].

[52] F. Lizzi, P. Vitale and A. Zampini, The Fuzzy Disc, J. High Energy Phys. 0308 (2003) 057 [hep-th/0306247]; From the Fuzzy Disc to Edge Currents in Chern-Simons Theory, Mod. Phys. Lett. A18 (2003) 2381 [hep-th/0309128].

[53] J. Madore, An Introduction to Noncommutative Differential Geometry and its Physical Applications (Cambridge University Press, 1995).

[54] G. Mandal, S.-J. Rey and S.R. Wadia, Quantum Aspects of GMS Solutions of Noncommutative Field Theory and Large $N$ Limit of Matrix Models, Eur. Phys. J. C24 (2002) 495 [hep-th/0111059].

[55] E.J. Martinec, The Annular Report on Noncritical String Theory, hep-th/0305148.

[56] E.J. Martinec and G. Moore, Noncommutative Solitons on Orbifolds, hep-th/0101199.

[57] J. McGreevy and H. Verlinde, Strings from Tachyons: The c = 1 Matrix Reloaded, J. High Energy Phys. 0312 (2003) 054 [hep-th/0304224].

[58] J. McGreevy, J. Teschner and H. Verlinde, Classical and Quantum DBranes in 2D String Theory, hep-th/0305194.

[59] A. Mikhailov, Non-Spherical Giant Gravitons and Matrix Theory, hep-th/0208077.

[60] S. Minwalla, M. Van Raamsdonk and N. Seiberg, Noncommutative Perturbative Dynamics, J. High Energy Phys. 0002 (2000) 020 [hep-th/9912072].

[61] G.J. Murphy, $C^{*}$-Algebras and Operator Theory (Academic Press, 1990).

[62] L.D. Paniak and R.J. Szabo, Instanton Expansion of Noncommutative Gauge Theory in Two Dimensions, Commun. Math. Phys. 243 (2003) 343 [hep-th/0203166].

[63] L.D. Paniak and R.J. Szabo, Lectures on Two-Dimensional Noncommutative Gauge Theory 2. Quantization, hep-th/0304268.

[64] J.-H. Park, Supersymmetric Objects in the M-Theory on a pp-Wave, J. High Energy Phys. 0210 (2002) 032 [hep-th/0208161]. 
[65] M. Pimsner and D. Voiculescu, Imbedding the Irrational Rotation $C^{*}$ Algebra into an AF Algebra, J. Oper. Theory 4 (1980) 93.

[66] M. Pimsner and D. Voiculescu, Exact Sequences for K-Groups and ExtGroups of Certain Cross-Product $C^{*}$-Algebras, J. Oper. Theory 4 (1980) 201.

[67] A. Pinzul and A. Stern, Edge States from Defects on the Noncommutative Plane, Mod. Phys. Lett. A18 (2003) 2509 [hep-th/0307234].

[68] M.A. Rieffel, $C^{*}$-Algebras Associated with Irrational Rotations, Pacific J. Math. 93 (1981) 415.

[69] M.A. Rieffel, The Cancellation Theorem for Projective Modules over Irrational Rotation $C^{*}$-Algebras, Proc. London Math. Soc. 47 (1983) 1285 .

[70] M.A. Rieffel, Projective Modules over Higher-Dimensional Noncommutative Tori, Can. J. Math. 40 (1988) 257.

[71] E.M. Sahraoui and E.H. Saidi, Solitons on Compact and Noncompact Spaces in Large Noncommutativity, Class. Quant. Grav. 18 (2001) 3339 [hep-th/0012259].

[72] N. Seiberg and E. Witten, String Theory and Noncommutative Geometry, J. High Energy Phys. 9909 (1999) 032 [hep-th/9908142].

[73] A. Sen, Some Issues in Noncommutative Tachyon Condensation, J. High Energy Phys. 0011 (2000) 035 [hep-th/0009038].

[74] H. Steinacker, Quantized Gauge Theory on the Fuzzy Sphere as Random Matrix Model, Nucl. Phys. B679 (2004) 66 [hep-th/0307075].

[75] R.J. Szabo, Quantum Field Theory on Noncommutative Spaces, Phys. Rept. 378 (2003) 207 [hep-th/0109162].

[76] S. Walters, The AF Structure of Noncommutative Toroidal $\mathbb{Z} / 4 \mathbb{Z}$ Orbifolds, math.0A/0207239.

[77] N.E. Wegge-Olsen, K-Theory and $C^{*}$-Algebras (Oxford Science Publications, 1993).

[78] E. Witten, Noncommutative Tachyons and String Field Theory, hep-th/0006071. 